\title{
Um Método Probabilístico em Combinatória
}

\section{César Alberto Bravo Pariente}

\author{
DISSERTAÇÃO APRESENTADA
}

$\mathrm{AO}$

INSTITUTO DE MATEMÁTICA E ESTATÍSTICA

DA

UNIVERSIDADE DE SÃO PAULO

PARA

OBTENÇÃO DO GRAU DE MESTRE

EM

MATEMÁTICA APLICADA

Área de Concentração: Ciência da Computação

Orientador : Prof. Dr. Yoshiharu Kohayakawa

-São Paulo, Outubro de 1996- 


\section{Um Método Probabilístico em Combinatória}

Este exemplar corresponde à redação final da dissertação devidamente corrigida e defendida por César Alberto Bravo Pariente e aprovada pela comissão julgadora.

São Paulo, 4 de Dezembro de 1996.

Banca examinadora:

- Prof. Dr. Yoshiharu Kohayakawa (orientador) - IME-USP

- Prof. Dr. Vladimir Belitsky - IME-USP

- Prof. Dr. David Grable - Humbolt Universität zu Berlin, Alemanha 


\section{Agradecimentos}

Sem dúvidas, o presente trabalho não tivesse podido ser realizado sem a direção do meu orientador, Professor Doutor Yoshiharu Kohayakawa. Sua paciência e apoio constante sempre foram além das minhas maiores expectativas. Sào para ele os protestos de meu maior respeito e agradecimento. É oportuno agradecer aqui também aos profesores Doutor Vladimir Belitsky e Doutor David Grable que fizeram parte de banca na defesa de minha dissertação.

Desejo agradecer também aos colegas que tiveram o trabalho de revisar tanto meu projeto de dissertação quanto as versões prévias deste trabalho, por ter me dedicado parte de seu tempo no meio de suas próprias atividades acadêmicas; notadamente Claus Akira Matsushigue, Flavio Keidi Miyazawa, Jair Donadelli Junior, Orlando Lee e Patricia Cristina Gimenez.

Quero ainda lembrar dos grandes amigos que fiz no Brasil, com quens compartilhe momentos bons e ruins, e que sempre me ajudaram a enfrentar os problemas que se apresentaram na minha estadia em Sampa: Flavio Keidi Miyazawa, Marcelo Esteban Coniglio, Simone, Sergio e Gabriel Borger, Sonia Elizabeth Trepode, os amigos do CRUSP e, ultimamente, a muito amigável turma '96 da Oficina de Teatro do TUSP.

Finalmente queria agradecer a todos meus professores, tanto da graduação na Universidad Nacional Mayor de San Marcos no Perú, quanto da pós graduação na Universidade de São Paulo no Brasil. Quero dedicar a eles (e elas) este trabalho, com a esperança de que nosso amor pela matemática seja tão duradouro (já que não tão frutífero), quanto o seu.

Durante a fase de créditos de matérias do mestrado o autor usufruiu de uma bolsa da CAPES. 


\section{Abstract}

The following work is an effort to present, in survey form, a collection of results that illustrate the application of a certain probabilistic method in combinatorics. We do not present new results in the area; however, we do believe that the systematic presentation of these results can help those who use probabilistic methods comprehend this useful technique.

The results we refer to have appeared over the last decade in the research literature and were used in the investigation of problems which have resisted other, more classical, approaches. Instead of theorizing about the method, we adopted the strategy of presenting three problems, using them as practical examples of the application of the method in question. Surprisingly, despite the difficulty of the solutions to these problems, they share the characteristic of being able to be formulated very intuitively, as we will see in Chapter One.

We should warn the reader that despite the fact that the problems which drive our discussion belong to such different fields as number theory, geometry, and combinatorics, our goal is to place emphasis on what their solutions have in common and not on the subsequent implications that these problems have in their respective fields. Occasionally, we will comment on other potential applications of the tools utilized to solve these problems.

The problems which we are discussing can be characterized by the decades-long wait for their solution: the first, from number theory, arose from the research in Fourier series conducted by Sidon at the beginning of the century and was proposed by him to Erdös in 1932. Since 1950, there have been diverse advances in the understanding of this problem, but the result we talk of comes from 1981. The second problem, from geometry, is a conjecture formulated in 1951 by Heilbronn and finally refuted in 1982. The last problem, from combinatorics, is a conjecture formulated by Erdös and Hanani in 1963 that was treated in several particular cases but was only solved in its entirety in 1985. 


\section{Prefacio}

O presente trabalho é um esforço de apresentar, organizado em forma de survey, um conjunto de resultados que ilustram a aplicação de um certo método probabilístico. Embora não apresentemos resultados novos na área, acreditamos que a apresentação sistemática destes resultados pode servir para a compreensão de uma ferramenta útil para quem usa dos métodos probabilísticos na sua pesquisa em combinatória.

Os resultados de que falaremos tem aparecido na última década na literatura especializada e foram usados na investigação de problemas que resistiram a outras aproximações mais clássicas. Em vez de teorizar sobre o método a apresentar, nós adotaremos a estratégia de apresentar três problemas, usando-os como exemplos práticos da aplicação do método em questão. Surpreendentemente, apesar da dificuldade que apresentaram para ser resolvidos, estes problemas compartilham a característica de poder ser formulados muito intuitivamente, como veremos no Capítulo 1.

Devemos advertir que embora os problemas que conduzem nossa exposição pertençam a áreas tão diferentes quanto teoria de números, geometria e combinatória, nosso intuito é fazer ênfase no que de comum tem as suas soluções e não das posteriores implicações que estes problemas tenham nas suas respectivas áreas. Ocasionalmente comentaremos sim, outras possíveis aplicações das ferramentas usadas para solucionar estes problemas de motivação.

Os problemas de que tratamos tem-se caracterizado por aguardar várias décadas em espera de solução: O primeiro, da teoria de números, surgiu na pesquisa de séries de fourier que Sidon realizava a princípios do século e foi proposto por ele a Erdős em 1932. Embora tenham havido, desde 1950, diversos avanços na pesquisa deste problema, o resultado de que falaremos data de 1981. Já o segundo problema, da geometria, é uma conjectura formulada em 1951 por Heilbronn e refutada finalmente em 1982. O último problema, de combinatória, é uma conjectura de Erdős e Hanani de 1963, que foi tratada em diversos casos particulares até ser finalmente resolvida em toda sua generalidade em 1985. 


\section{Sumário}

1 Visita guiada à motivação e notações $\quad 2$

1.1 Uma seqüência de Sidon densa . . . . . . . . . . . . . . . . . . 4

1.1.1 O número de independência dos grafos sem triângulos . . . . . . . 8

1.2 Um limitante inferior para o problema de Heilbronn . . . . . . . . . . . . 10

1.2.1 O número de independência dos hipergrafos sem 2-circuitos . . . . . 11

1.3 A conjectura de Erdős e Hanani . . . . . . . . . . . . . . . . . . . . 13

1.3.1 A versão restrita de Pippenger do teorema de Frankl e Rödl . . . . 14

2 Conjuntos Independentes 18

2.1 Grafos sem triângulos . . . . . . . . . . . . . . . . . 18

2.1.1 A transformação . . . . . . . . . . . . . . 18

2.1 .2 O algoritmo . . . . . . . . . . . . . . . . . 30

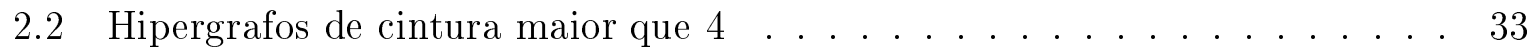

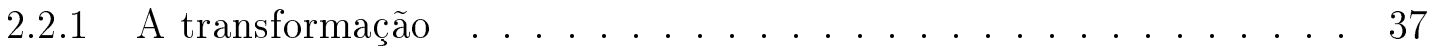

2.2.2 O algoritmo . . . . . . . . . . . . . . . . . 41

2.3 Conseqüências . . . . . . . . . . . . . . . . . . . . . . . . . . . . . . . . . . . . . 43

2.3.1 Uma seqüência de Sidon densa . . . . . . . . . . . . . . . 43

2.3.2 Um limitante inferior para o problema de Heilbronn . . . . . . . . . 49

3 Uma Técnica de Rödl

3.1 A versão restrita de Pippenger do teorema de Frankl e Rödl . . . . . . . . 53

3.1 .1 A transformação . . . . . . . . . . . . . . . . 53

3.1 .2 O algoritmo . . . . . . . . . . . . . . . . . . 59

3.2 Conseqüências: a conjectura de Erdős e Hanani . . . . . . . . . . . . . . . 61

3.3 Apêndice: Desenvolvimento histórico da técnica de Rödl . . . . . . . . . . . 63

3.3.1 O trabalho seminal de Rödl e os comentários de Spencer . . . . . . 63

3.3.2 O teorema de Frankl e Rödl . . . . . . . . . . . . . . . . . 67

3.3.3 A extensão de Pippenger e Spencer . . . . . . . . . . . . . . 69 


\section{Capítulo 1}

\section{Visita guiada à motivação e notações}

Esta introdução pretende ser um roteiro para a leitura de nossa dissertação. Vamos apresentar aqui os problemas que motivaram os resultados expostos em nosso trabalho e pretendemos que esta apresentação seja o mais "reader-friendly" possível para que o leitor possa usá-la mesmo para se informar rapidamente de nossa discussão, usando os capítulos seguintes para conhecer os detalhes das demonstrações.

Adotamos esta estratégia mesmo porque o discurso nos capítulos seguintes não é convidativo o suficiente para permitir uma rápida revisão do material apresentado.

Este é o momento para explicitar o que são os três problemas "leit-motiv" de que falaremos: o primeiro, da teoria de números, consiste na construção de uma seqüência pertencente a uma certa classe. O segundo, da geometria, limita inferiormente a área mínima num certo tipo de configuração de triângulos no plano. O terceiro é um problema de "combinatória pura", por assim dizer, e preferimos adiar um pouco sua descrição.

Para ilustrar o uso do método probabilístico nestes problemas de motivação, nós nos concentraremos na discussão de dois objetos combinatórios, número de independência em grafos e hipergrafos, e coberturas mínimas, discutidos cada um deles nos Capítulos 2 e 3 respectivamente.

Em cada caso, apresentaremos o problema de motivação de forma intuitiva e assinalaremos as distintas etapas que se deram na sua pesquisa, remetendo o leitor à seção correspondente da dissertação para os detalhes técnicos das provas.

Vamos esboçar agora o método probabilístico que está por trás dos avanços na pesquisa dos problemas mencionados.

Em geral, o uso de técnicas probabilísticas em combinatória permite mostrar a existência de um certo objeto combinatório. A idéia geral é mostrar que a probabilidade de que exista um objeto como desejado é positiva. Por exemplo, num grafo, podemos estar interessados em mostrar a existência de um conjunto "grande" de vértices entre os quais não há arestas.

A estratégia trivial para uma tal demonstração existencial consiste em sortear independentemente, com alguma probabilidade, os elementos do conjunto envolvido (no exemplo, os vértices) e calcular depois a probabilidade de que os elementos escolhidos formem o ob- 
jeto combinatório desejado. Se essa probabilidade for positiva, podemos ficar satisfeitos e aceitar esta estratégia como razoável para a construção do objeto desejado. Mas, em geral, essa pode ser uma visão otimista demais.

Em vez disso, a estratégia do método probabilístico que visamos ilustrar neste trabalho consiste em construir aos poucos o objeto combinatório no qual estamos interessados: ou seja, escolhe-se uma quantidade pequena dos elementos do conjunto de interesse de modo a formar um objeto como o desejado, mas de tamanho menor. Depois repete-se esta construção um número suficiente de vezes de modo que a união dos objetos "pequenos" construídos a cada passo formem o objeto combinatório do tamanho desejado. A desvantagem desta aproximação de "sorteios sucessivos" é a variação indesejada de alguns parâmetros de interesse. Por exemplo, se num grafo todos os vértices incidem em três arestas podemos perder essa propriedade ao apagar alguns vértices.

Tencionamos ressaltar que as provas dos resultados principais esboçadas neste trabalho compartilham de características semelhantes: em linhas gerais, essas provas dividem-se em duas etapas, a que nós nos referiremos como "A transformação" e "O algoritmo". Na primeira etapa, estuda-se uma transformação do objeto em consideração, transformação esta que altera os parâmetros do objeto de forma controlada; tipicamente, a transformação envolve escolher e remover um conjunto (de vértices ou arestas) ao acaso. Na segunda etapa, mostra-se que iterando-se a transformação em questão um número adequado de vezes, pode-se fazer com que os parâmetros de interesse atinjam um determinado valor.

A seguir vamos expor o funcionamento deste processo em cada um dos problemas que trataremos. 


\section{$1.1 \quad$ Uma seqüência de Sidon densa}

Nesta seção vamos discorrer sobre um certo problema da teoria de números que Sidon propôs em 1932 a Erdős. Trataremos sempre de seqüências formadas por números naturais. Em geral, pode se dizer que neste contexto é interessante conhecer a rapidez de crescimento das seqüências em estudo. Sendo pouco relevante estudar essa rapidez para seqüências particulares, vamos nos concentrar em seqüências pertencentes a uma certa "classe". Para dar um pouco de contexto ao título desta seção vamos definir o conceito de densidade de uma seqüência, embora ele esteja presente só implicitamente nos resultados que vamos descrever.

Definição 1.1.1 Seja $S$ uma seqüência de números inteiros positivos estritamente monótona crescente, digamos $S=\left\{a_{1}<a_{2}<\ldots<a_{k}<\ldots\right\}$, finita ou infinita. Para cada $n$ definimos o número de contagem $f_{S}(n)$ de $S$ até $n$ (também conhecido por função de contagem) como o número de elementos de $S$ menores ou iguais a $n$.

$A$ densidade de Schnirelmann $\sigma S$ de $S$ é

$$
\sigma S=\inf _{n} \frac{f_{S}(n)}{n}
$$

$A$ densidade assintótica inferior $\underline{\mathbf{d}}$ de $S$ e a densidade assintótica superior $\overline{\mathbf{d}}$ de $S$ são definidas por

$$
\begin{aligned}
& \underline{\mathbf{d}} S=\liminf _{n \rightarrow \infty} \frac{f_{S}(n)}{n}, \\
& \overline{\mathbf{d}} S=\limsup _{n \rightarrow \infty} \frac{f_{S}(n)}{n} .
\end{aligned}
$$

$S e \underline{\mathbf{d}} S=\overline{\mathbf{d}} S$ dizemos que $S$ possui densidade assintótica $\mathbf{d} S$, dada pelo valor comum.

Segundo Erdős (cf. [7]), Sidon colocou três problemas:

(1) Seja $S=\left\{a_{1}<a_{2}<\ldots<a_{k}<\ldots\right\}$ uma seqüência infinita de inteiros e, para todod $n$ inteiro positivo, $B_{S}(n)$ o número de soluções de $n=a_{i}+a_{j}$. Existe uma seqüência $S$ para a qual $B_{S}(n)>0$, porém para todo $\epsilon>0$ temos $B_{S}(n) / n^{\epsilon} \rightarrow 0$, conforme $n \rightarrow \infty$ ?

(2) Seja $S=\left\{1 \leq a_{1}<a_{2}<\ldots<a_{k} \leq n\right\}$ e suponha que todos os $a_{i}+a_{j}$ são diferentes para todo $i, j \in\{1, \ldots, k\}$. Determine $f_{S}(n)=\max k$.

(3) Seja $S=\left\{a_{1}<a_{2}<\ldots<a_{k} \ldots\right\}$ e suponha que todos os $a_{i}+a_{j}$ são diferentes para todo $i, j \in\{1, \ldots, k, \ldots\}$. Determine $f_{S}(n)$. 
Observe que uma seqüência satisfazendo (1) é uma base dos naturais no sentido que todo natural pode-se escrever como soma de seus elementos. Por outro lado, as seqüências satisfazendo (2) e (3) não precisam ter esta propriedade e, mais ainda, a proibição de repetir somas impede obter alguns dos inteiros como soma de elementos de seqüências destes tipos. Nós nos concentraremos em seqüências de tipo (2) e (3).

A pesquisa desenvolvida em torno destes problemas tem dado origem a uma área conhecida como teoria aditiva de números. Em homenagem a Sidon, as seqüências como as descritas por (2) e (3) formam uma classe que leva seu nome.

Definição 1.1.2 Uma seqüência de Sidon é uma seqüência de números inteiros positivos $S=\left\{a_{1}<a_{2}<\ldots<a_{k}<\ldots\right\}$, finita ou infinita, tal que todo inteiro $m$ tem, no máximo, uma representação da forma

$$
m=a_{i}+a_{j}, \quad \operatorname{com} a_{i}, a_{j} \in S, \quad i<j .
$$

Por abuso de notação, vamos às vezes chamar as seqüências de Sidon de conjuntos de Sidon.

Vamos considerar primeiro o caso finito.

Denotamos com $s(n)$ o número máximo de elementos de $[n]=\{1,2, \ldots, n\}$ que formam uma seqüência de Sidon. Os primeiros limites triviais para $s(n)$ são os seguintes:

$$
(2 n)^{1 / 3}<s(n)<(2 n)^{1 / 2}+1,
$$

sendo que o limite inferior é fornecido pelo algoritmo guloso e o superior é devido ao fato de que para toda seqüência de Sidon $S$ contida em $[n]$, as diferenças positivas dos elementos de $S$ são números distintos menores que $n$.

O problema de determinar $s(n)$ foi investigado por Erdős, Turán e Chowla com os seguintes resultados.

Teorema 1.1.3 (Erdős e Turán, '41; [9])

$$
\frac{1}{\sqrt{2}} \leq \liminf \frac{s(n)}{\sqrt{n}} \leq \limsup \frac{s(n)}{\sqrt{n}} \leq 1 .
$$

Teorema 1.1.4 (Erdős, '44; Chowla, '44; [6] e [4])

$$
\liminf \frac{s(n)}{\sqrt{n}} \geq 1
$$

Ou seja, o crescimento de $s(n)$ é aproximadamente o crescimento da raiz quadrada de $n$, conforme $n$ cresce indefinidamente. 
No caso infinito não se tem resultados tão precisos. Seja $S$ uma seqüência de Sidon infinita, denotamos por $f_{S}(n)$ o número de elementos de $S$ não maiores que $n$. Em [9], Erdős e Turán comentam que para toda seqüência infinita de Sidon $S$ temos

$$
\liminf \frac{f_{S}(n)}{\sqrt{n}}=0,
$$

mas que existe uma seqüência de Sidon $S$ tal que

$$
\limsup \frac{f_{S}(n)}{\sqrt{n}} \geq \frac{1}{2} .
$$

De fato, para a primeira afirmativa, Erdős provou muito mais:

Teorema 1.1.5 (Erdős, '55; [12]) Para toda seqüência infinita de Sidon S temos

$$
\liminf f_{S}(n) \sqrt{\frac{\log n}{n}}<\infty .
$$

Temos ainda uma melhora para o limite superior.

Teorema 1.1.6 (Krückenberg, '61; [15]) Existe uma seqüência infinita de Sidon S tal que

$$
\lim \sup \frac{f_{S}(n)}{\sqrt{n}} \geq \frac{1}{\sqrt{2}} .
$$

Já que tanto limite inferior quanto limite superior caracterizam subseqüências convergentes aos pontos de acumulação mínimo e máximo da seqüência original, esses resultados não dizem muito sobre a rapidez de crescimento de $f_{S}(n)$.

Passaram-se vinte anos até se conseguir construir uma seqüência de Sidon de crescimento mais rápido: em 1981, Ajtai, Komlós e Szemerédi [2] apresentaram uma construção probabilística de uma seqüência de Sidon mais densa que as anteriores. Eis o enunciado.

Teorema 1.1.7 (Ajtai, Komlós e Szemerédi, '81; [2]) Existe uma seqüência de Sidon $S$ tal que

$$
f_{S}(n)>c(n \log n)^{1 / 3},
$$

para todo $n \in \mathrm{N}$, onde $c>0$ é uma constante universal.

A estratégia geral na prova dos resultados anteriores ao Teorema 1.1.7 esteve baseada em dois fatos:

(a) Associar a cada número primo $p$ um conjunto finito de Sidon, normalmente baseado em congruências módulo $p$. 
(b) Escolher uma seqüência de números primos tal que os conjuntos associados a primos diferentes não compartilhem somas.

Desse modo a união dos conjuntos associados aos números primos da seqüência formava a seqüência infinita de Sidon procurada.

Existia neste enfoque um compromisso na construção da seqüência de primos. Por um lado, era necessário que termos consecutivos desta seqüência estivessem longe o bastante para evitar que seus conjuntos associados compartilhassem somas. Por outro lado, os tais termos consecutivos não deviam estar longe demais para evitar que a densidade da seqüência fosse "baixa". Manter esse compromisso foi a preocupação principal de Erdős, Turán e Krückenberg.

No novo resultado, Ajtai, Komlós e Szemerédi, atacam este problema construindo a tal seqüência de primos baseando-se num resultado de grafos sem triângulos (3-circuitos; veja a próxima subseção, Teorema 1.1.9). A idéia básica é usar a proibição de existir triângulos para evitar repetir somas de termos da seqüência em construção. Os conjuntos associados aos números primos também são melhorados.

Antes de abandonar a discussão do Teorema 1.1.7 vamos mencionar uma outra aplicação sua que tem recuperado importância devido a um resultado recente na investigação dos números de Ramsey $R(3, t)$.

O grafo completo $K^{n}$ é o grafo sobre $n$ vértices tal que entre quaisquer par de vértices existe uma aresta. Se $s, t$ são inteiros positivos, definimos o número de Ramsey $R(s, t)$ como o mínimo $n$ tal que toda 2-coloração das arestas de $K^{n}$ induz um $K^{s}$ monocromático ou um $K^{t}$ monocromático. Um subgrafo completo é também chamado de clique. Equivalentemente então, $R(s, t)$ é o menor inteiro tal que todo grafo sobre $n$ vértices tem um clique de $s$ vértices ou um conjunto independente de $t$ vértices. Usamos $G_{n}^{(3)}$ para denotar um grafo livre de $K^{3}$, ou seja, tal que nenhum três de seus vértices induz um $K^{3}$. Em particular temos

$$
R(3, t)=\min \left\{n: \alpha\left(G_{n}^{(3)}\right) \geq t \text { para todo } G_{n}^{(3)}\right\} .
$$

Considerando os grafos $G_{n}^{(3)}$ com grau máximo $t$ temos que o Teorema 1.1.7 implica trivialmente que vale

$$
n=R(3, t) \leq c_{1} t^{2} / \log t
$$

Recentemente Kim [13], tem mostrado que

$$
c_{2}(1-o(1)) t^{2} / \log t \leq R(3, t)
$$

provando assim que o ordem de magnitude de $R(3, t)$ é justamente $t^{2} / \log t$. A prova do resultado de Kim está baseada no "método da semialeatoridade" desenvolvido a partir do trabalho de Rödl que comentamos na Seção 3.3.1 


\subsubsection{O número de independência dos grafos sem triângulos}

Definição 1.1.8 Um grafo $G=(V G, E G)$ é formado por um conjunto $V G$ de vértices e um conjunto EG de arestas.

Cada aresta une um par de vértices.

Dado um vértice $x$ o número de vértices aos quais $x$ está unido é o grau $d(x)$ de $x$.

$O$ conjunto dos vértices aos quais $x$ está unido é chamado vizinhança de $x$ e denotado por $\Gamma(x)$.

$O$ grau médio $t=t(G)$ do grafo $G$ é definido por $t=\sum_{x \in V G} d(x) / n$.

Um conjunto independente (de vértices) de $G$ é um subconjunto de $V G$ entre os quais não há arestas.

$O$ número de independência $\alpha(G)$ do grafo $G$ é o tamanho de um conjunto independente máximo, i.e., tal que não existe outro conjunto independente de cardinalidade maior.

Se $S \subset V$ definimos o subgrafo induzido por $S$, denotado $G[S]$, como o grafo com conjunto de vértices $S$ e conjunto de arestas

$$
E G[S]=\{x y \in E G: x, y \in S\} .
$$

No Capítulo 2 estuda-se o número de independência (isto é, o tamanho de um conjunto máximo de vértices que não induz arestas) no contexto de grafos e hipergrafos; o resultado central para grafos é o seguinte.

Teorema 1.1.9 (Ajtai, Komlós e Szemerédi, '81; [2]) Se um grafo G livre de triângulos com $n$ vértices tem grau médio $t>c_{1}$, então $G$ tem número de independência

$$
\alpha(G)>c_{2} n(\log t) / t
$$

onde $c_{1}>0$ e $c_{2}>0$ são constantes absolutas.

Para definir a transformação neste caso, mostra-se que se pode tomar um conjunto de vértices $K$ de $G$ ao acaso que induz um subgrafo com número de independência grande e define-se o grafo transformado $G^{*}$ como o grafo induzido pelos vértices que não induzem arestas com vértices de $K$. O conjunto $K$ deve ser tal que o quociente $n^{\prime} / t^{\prime}$ entre o número de vértices $n^{\prime}$ do grafo transformado e seu grau médio $t^{\prime}=\sum_{x \in V G^{*}} d(x) / n^{\prime}$ não decresce muito em relação ao quociente do grafo original. Note que este quociente é essencialmente o limite inferior para $\alpha(G)$ do Teorema 1.1.9. Para completar a prova deste resultado, iteramos a transformação descrita até obtermos um grafo transformado com um número desprezível de vértices em relação ao grafo original. Demonstra-se a seguir que a união dos conjuntos independentes máximos dos subgrafos induzidos pelos conjuntos $K$ gerados durante as iterações formam um conjunto independente de tamanho suficientemente grande em $G$. 
Também no Capítulo 2, apresentamos um resultado análogo ao Teorema 1.1.9 para hipergrafos. Este resultado é, entretanto, mais técnico e assim não o discutiremos aqui. Os resultados acima foram concebidos como instrumentos na investigação de problemas combinatórios em teoria de números e geometria (cf. Seções 2.3.1 e 2.3.2). 


\subsection{Um limitante inferior para o problema de Heil- bronn}

Consideremos o disco $D$ de área 1 no plano e um conjunto $S=\left\{p_{1}, \ldots, p_{n}\right\}$ de $n$ pontos de $D$. Seja $\Delta(S)$ a área mínima de um triângulo cujos vértices são 3 pontos diferentes de $D$. Heilbronn $(\leq 51)$ colocou o problema de calcular

$$
\Delta(n)=\max \Delta(S)
$$

onde $S$ toma valores sobre todos os conjuntos de $n$ pontos em $D$. A primeira estimativa para $\Delta(n)$ é

$$
\Delta(n)=O\left(n^{-1}\right),
$$

e Heilbronn conjecturou que o valor correto era $\Delta(n)=O\left(n^{-2}\right)$.

Conjectura 1.2.1 (Heilbronn, $\leq$ '51) Para alguma constante absoluta $c>0$, toda configuração de $n$ pontos no disco unitário contém necessariamente um triângulo de área menor que $c / n^{2}$.

Erdős mostrou [18] que se essa conjectura fosse válida então o resultado seria o "melhor possível". Para tanto ele provou que

$$
\Delta(n) \neq o\left(n^{-2}\right)
$$

construindo um conjunto de $n$ pontos em $n^{-1}\left([1, n]^{2} \cap \mathrm{Z}^{2}\right)$ nenhum três deles colineares; a afirmativa decorre de que todo triângulo nesse reticulado tem área $\geq c / n^{2}$, para alguma constante absoluta $c$.

Vamos comentar como a visão probabilística "clássica" fornece apenas

$$
\Delta(n) \geq c n^{-2}
$$

Com efeito, dado $\epsilon>0$, pode-se limitar por $c \epsilon$ a probabilidade de que 3 pontos de $D$ escolhidos uniforme e independentemente formem um triângulo de área $\leq \epsilon$.

Tomando $\epsilon=c / n^{2}$ pode-se provar que $2 n$ pontos escolhidos ao acaso uniforme e independentemente definem em média no máximo $n$ triângulos de área $<c / n^{2}$. Retirando um vértice de cada um desses triângulos, obtemos um conjunto $S$ de pelo menos $n$ pontos de $D$ sem triângulos de área menor que $c / n^{2}$.

Porém a conjectura de Heilbronn é falsa e foi refutada em 1982 por Komlós, Pintz e Szemerédi, [14], que provaram que $\Delta(n)$ não só não é dominada por $c / n^{2}$ (para alguma constante $c$ ), como seu crescimento é, pelos menos, mais veloz que esse limite vezes um fator logarítmico. 
Teorema 1.2.2 (Komlós, Pintz e Szemerédi, '82; [14]) Para todo inteiro $n$ existe uma configuração de $n$ pontos no disco unitário que não contém triângulos de área menor que

$$
c_{1}(\log n) / n^{2},
$$

onde $c_{1}>0$ é uma constante absoluta.

A construção probabilística que prova este teorema é uma bela amostra da aplicação de resultados combinatórios em geometria. O coração da prova consiste em definir um 3grafo $\mathcal{F}$ sobre $n^{1+\epsilon}$ pontos escolhidos ao acaso no disco unitário, pondo três de tais pontos numa aresta de $\mathcal{F}$ se eles formam um triângulo de área menor ou igual a $c_{1}(\log n) / n^{2}$. Depois mostra-se que retirando-se um vértice de cada um dos circuitos de comprimento dois de $\mathcal{F}$, o número de vértices não diminui sensivelmente. Daí o Teorema 1.2.5 (veja a próxima subseção) com parâmetro $k=2$ fornece um conjunto independente de tamanho $n$ em $\mathcal{F}$, i.e., $n$ pontos que não contém triângulos de área menor que $c_{1}(\log n) / n^{2}$, provando assim o Teorema 1.2.2.

\subsubsection{O número de independência dos hipergrafos sem 2-circuitos}

Definição 1.2.3 Um hipergrafo $\mathcal{F}=(X, \mathcal{F})$ sobre um conjunto não vazio $X$ é uma família de subconjuntos de $X$.

Os elementos de $X$ são os vértices $e$ os elementos de $\mathcal{F}$ são as hiperarestas de $\mathcal{F}$.

$O i$-grau $d_{i}(x)$ de um vértice $x \in X$ é o número de $(i+1)$-hiperarestas contendo $x$, i.e. a quantidade de hiperarestas de tamanho $i+1$ que contém $x$.

$A$ vizinhança de $x$, denotada $\Gamma(x)$, é o conjunto dos vértices que pertencem a alguma hiperaresta que contém o vértice $x$. Definimos, ademais,

$$
\begin{aligned}
& \Gamma^{1}(x)=\Gamma(x) \\
& \Gamma^{i}(x)=\bigcup_{y \in \Gamma^{i-1}(x)} \Gamma(y), \quad .
\end{aligned}
$$

Se todas as hiperarestas tem o mesmo tamanho $h$, dizemos que $\mathcal{F}$ é um h-grafo.

$U m$ conjunto independente (de vértices) de $\mathcal{F}$ é um subconjunto de $X$ que não contém nenhuma hiperaresta de $\mathcal{F}$.

$O$ número de independência $\alpha(\mathcal{F})$ do hipergrafo $\mathcal{F}$ é o tamanho de um conjunto independente máximo de $\mathcal{F}$, i.e., tal que não existe outro conjunto independente de cardinalidade maior.

Se $S \subset X$ definimos o subhipergrafo induzido por $S$, denotado $\mathcal{F}[S]$, como o grafo com conjunto de vértices $S$ e conjunto de hiperarestas

$$
\mathcal{F}[S]=\{F \in \mathcal{F}: F \subset S\} .
$$


Definição 1.2.4 Dado um hipergrafo $\mathcal{F}=(V, \mathcal{F})$ e $X \subset V$, pomos

$$
\mathcal{F}(X)=\{F \in \mathcal{F}: X \subset F\},
$$

$e$

$$
d(X)=|\mathcal{F}(X)| .
$$

Quando $X=\{x\}$, escrevemos simplesmente $\mathcal{F}(x)$ e $d(x)$ e se $X=\{x, y\}$ escrevemos $\mathcal{F}(x, y)$ ed $(x, y)$.

Como acabamos de comentar, a prova do Teorema 1.2.2 está baseada no teorema seguinte.

Teorema 1.2.5 (Duke, Lefmann e Rödl, '95; [5]) Sejam dados um inteiro $k \geq 2 e$ um $(k+1)$-grafo $\mathcal{F}$ sobre $n$ vértices tais que para algum $t_{0}=t_{0}(n)$ e algum $n_{0}=n_{0}(k, t)$ temos

(i) duas hiperarestas quaisquer se intersectam no máximo num vértice,

(ii) $\Delta(\mathcal{F}) \leq t^{k}$, onde $t \geq t_{0}(k)$,

(iii) $n \geq n_{0}(k, t)$.

Então

$$
\alpha(\mathcal{F}) \geq c_{k} \frac{n}{t}(\log t)^{1 / k},
$$

onde $c_{k}$ é uma constante absoluta.

Na verdade, este teorema é um corolário de um teorema com hipóteses mais restritivas devido a Ajtai, Komlós, Pintz, Spencer e Szemerédi [1]. Essa versão estabelece o limitante inferior do Teorema 1.2.5 para hipergrafos sem circuitos de comprimento 2, 3 e 4 . A sua prova foi também baseada no método probabilístico que estamos apresentando. No breve preâmbulo à Subseção 2.2.1 apresentaremos a dedução deste resultado mais geral a partir da versão mais restrita. Na Seção 2.2 apresentaremos, com algum detalhe, o roteiro que segue a demonstração do resultado de Ajtai, Komlós, Pintz, Spencer e Szemerédi, embora não apresentemos as provas em forma completa. 


\subsection{A conjectura de Erdős e Hanani}

O problema que motiva esta seção é um pouco diferente dos anteriores no sentido que é um problema abstrato de combinatória. Embora seu enunciado seja muito intuitivo, os sucessivos avanços na técnica usada na prova original permitem hoje dizer que a prova original foi desnecessariamente complicada. Eis o enunciado original.

Dados inteiros $r, k$ e $n(r \leq k \leq n)$, definimos $M(k, r, n)$ como a cardinalidade de um sistema mínimo de $k$-conjuntos (subconjuntos de tamanho $k$ ) de $[n]=\{1, \ldots, n\}$ tal que todo $r$-conjunto de $[n]$ está contido em, no mínimo, um dos $k$-conjuntos do sistema. Analogamente denotamos por $m(k, r, n)$ a cardinalidade de um sistema máximo de $k$ conjuntos de $[n]$ tal que todo $r$-conjunto de $[n]$ está contido, no máximo, num $k$-conjunto do sistema.

Conjectura 1.3.1 (Erdős e Hanani, '63; [8]) Para todo $r \leq k$ fixos, temos

$$
\lim _{n \rightarrow+\infty} m(k, r, n)\left(\begin{array}{l}
k \\
r
\end{array}\right)\left(\begin{array}{l}
n \\
r
\end{array}\right)^{-1}=\lim _{n \rightarrow+\infty} M(k, r, n)\left(\begin{array}{l}
k \\
r
\end{array}\right)\left(\begin{array}{l}
n \\
r
\end{array}\right)^{-1}=1
$$

Erdős e outros (veja [11]) provaram diversos casos particulares desta conjectura. Porém, foi só em 1985 que Rödl [17], forneceu uma prova desta conjectura em toda sua generalidade. Ele provou a conjectura de Erdős e Hanani usando r-grafos completos para empacotar os r-conjuntos. No mesmo ano, Spencer [19] aprimorou o resultado de Rödl relaxando um pouco as hipoteses e introduzindo o conceito de hipergrafo pseudo aleatório com densidade $\rho$ e tolerância $\delta$. A cobertura dos r-conjuntos ainda se faz com r-grafos completos. Ainda no mesmo ano, Frakl e Rödl [10], conseguem impor condições suficientes para a existência de uma cobertura quase perfeita num r-grafo. Eles conseguem deixar de usar r-grafos completos para cobrir os r-conjuntos, retirando apenas um conjunto de arestas e definindo como hipergrafo transformado aquele formado pelas arestas que não se intersectam com as retiradas. As condicões de regularidade de Spencer são trocadas por condições sobre o grau dos vértices e sobre a quantidade de arestas contendo dois vértices. Embora este novo resultado represente um avanço importante na simplificação da técnica desenvolvida para provar a conjectura de Erdős e Hanani, ele ainda exige hipóteses um pouco restritivas (a quantidade de hiperarestas que contém dois vértices deve ser inversamente proporcional a uma potencia do logaritmo do número de vértices).

Finalmente, em '89 Pippenger e Spencer [16], apresentaram sua extensão do teorema de Frankl e Rödl, que já fora comentado em 1988 por Füredi [11], quem, ademais, apresentou a versão restrita de Pippenger no seu artigo. O resultado de Pippenger e Spencer [16] é mais geral que o de Frankl e Rödl no sentido que suas hipóteses são menos restritivas (a quantidade de hiperarestas que contém dois vértices é limitada linearmente pelo grau máximo do hipergrafo). Este novo resultado mostra que as famílias de r-grafos com grau mínimo assintótico ao grau máximo e tal que o número de hiperarestas contendo dois vertices quaisquer é desprezivel em relação ao grau máximo, tem-se que o índice cromático 
é assintótico ao grau máximo. Isto quer dizer que as hiperarestas podem ser particionadas em coberturas ou emparelhamentos quase perfeitos. É importante mencionar que o trabalho de Pippenger e Spencer tem dado origem a resultados de J. Kahn sobre outras conjecturas de Erdős. Nós mencionamos estes resultados no nosso projeto de dissertação porém temos abandonado a pretensão de tratá-los nesta versão final.

$\mathrm{Na}$ Seção 2.3 apresentamos o desenvolvimento histórico da técnica originada na prova desta conjectura. Aqui comentamos como essa conjectura é conseqüência do teorema de Frankl e Rödl na versão de Pippenger.

\subsubsection{A versão restrita de Pippenger do teorema de Frankl e Rödl}

Definição 1.3.2 Seja $\mathcal{F}$ um hipergrafo. Uma cobertura (de vértices por arestas) $\mathcal{K}$ em $\mathcal{F}$ é um conjunto de hiperarestas de $\mathcal{F}$ tal que cada vértice está, no mínimo, numa hiperaresta de $\mathcal{K}$. Um empacotamento $\mathcal{P}$ em $\mathcal{F}$ é um conjunto de hiperarestas de $\mathcal{F}$ tal que todo vértice está, no máximo, numa hiperaresta de $\mathcal{P}$. Uma cobertura ou um empacotamento é perfeito se cada vértice está em exatamente uma de suas hiperarestas. Neste caso os conceitos coincidem. Denotamos por $\nu(\mathcal{F})$ a cardinalidade de um empacotamento máximo e por $\operatorname{Cob}(\mathcal{F})$ a cardinalidade de uma cobertura minima de $\mathcal{F}$.

Dado um número inteiro positivo $n$, usamos $[n]$ para denotar o conjunto dos $n$ primeiros inteiros positivos, i.e., $[n]=\{1, \ldots, n\}$.

Se $n$ e $r$ são inteiros positivos tais que $r \leq n$, denotamos com $[n]^{(r)}$ a coleção dos subconjuntos de $[n]$ de tamanho r, i.e.,

$$
[n]^{(r)}=\{F \subset[n]:|F|=r\}
$$

Dados $A$ e $B$ números reais e $\epsilon>0$ usamos $A \sim_{\epsilon} B$ para denotar $1-\epsilon \leq A / B \leq 1+\epsilon$.

Vamos expressar agora a conjectura de Erdős e Hanani como o problema de achar a cobertura mínima de um certo hipergrafo.

Consideremos o $\left(\begin{array}{l}k \\ r\end{array}\right)$-grafo $\mathcal{F}$ com conjunto de vértices $V(\mathcal{F})=[n]^{(r)}$, e $F \in \mathcal{F}$ se e somente se $|\cup F|=k$ e $|F|=\left(\begin{array}{l}k \\ r\end{array}\right)$.

Observamos agora que a cardinalidade de uma cobertura mínima deste hipergrafo é exatamente $M(k, r, n)$. Para provar a segunda afirmativa da conjectura de Erdős e Hanani, bastaria então que conseguíssemos provar que este número é assintoticamente igual ao quociente entre o número de vértices do hipergrafo $\left(\begin{array}{l}n \\ r\end{array}\right)$ e o tamanho de suas arestas $\left(\begin{array}{l}k \\ r\end{array}\right)$. Isto é o que garante o seguinte teorema.

Teorema 1.3.3 (Pippenger, cf. Füredi, '88; [11]) Para todo inteiro $r \geq 2$ e números reais $K \geq 1$ e $\epsilon>0$ existe um $\delta>0$ para o qual vale o seguinte. Se, para algum $d$ inteiro positivo, o r-grafo $\mathcal{F}=(V, \mathcal{F})$ sobre $n$ vértices satisfaz 
(i) $d(x)<K d$ para todo $x \in V$,

(ii) $d(x) \sim_{\delta} d$ vale para, no mínimo, $(1-\delta) n$ dos vértices,

(iii) $d(x, y)<\delta d$ para todo $x \neq y$,

então

$$
\operatorname{Cob}(\mathcal{F})<(n / r)(1+\epsilon)
$$

Na Seção 3.2 mostraremos como ambas as afirmativas da conjectura de Erdős e Hanani, podem ser deduzidas deste resultado. Agora descreveremos como se aplica a estratégia geral do método probabilístico na prova deste teorema.

Começamos descrevendo o que significa a transformação neste caso e na continuação esboçamos a prova desta transformação, o que é equivalente a descrever uma iteração do algoritmo que prova o Teorema 1.3.3. Terminaremos a discussão deste teorema comentando como são escolhidos os parâmetros e os limites do referido algoritmo.

Lema 1.3.4 (A transformação) Sejam fixos $r, \epsilon>0$ e K. Então para todo $\delta^{\prime}>0$, existe um $\delta>0$ para o qual vale o seguinte. Se $n>n_{0}\left(r, \delta^{\prime}, \epsilon, K\right)$ e $D>D_{0}\left(r, \delta^{\prime}, \epsilon, K\right)$ e é dado um r-grafo $\mathcal{F}=(V, \mathcal{F})$ sobre $n$ vértices satisfazendo

(i) $d(x)<K D$ para todo $x \in V$,

(ii) $d(x) \sim_{\delta} D$ vale para, no mínimo, $(1-\delta) n$ vértices,

(iii) $d(x, y)<\delta D$ para todo $x \neq y$,

então existe $\mathcal{R} \subset \mathcal{F}$ tal que pondo $V^{*}=V \backslash \cup \mathcal{R}$, e $\mathcal{F}^{*}=\left.\mathcal{F}\right|_{V^{*}}$, temos

(iv) $|\mathcal{R}| \sim_{\delta^{\prime}} \epsilon n / r$,

(v) $\left|\cup \mathcal{F}^{*}\right| \sim_{\delta^{\prime}} n \mathrm{e}^{-\epsilon}$,

(vi) $d^{*}(x) \sim_{\delta^{\prime}} D \mathrm{e}^{-\epsilon(r-1)}$ vale para, no mínimo, $\left(1-\delta^{\prime}\right)\left|\cup \mathcal{F}^{*}\right|$ dos vértices de $\mathcal{F}^{*}$.

Passamos a esboçar a prova deste lema.

Escolhe-se um conjunto de hiperarestas pequeno e inversamente proporcional ao grau "predominante" $D$ do hipergrafo $\mathcal{F}$. Retira-se então do hipergrafo original todos os vértices pertencentes às hiperarestas escolhidas, obtendo-se assim o hipergrafo transformado $\mathcal{F}^{*}$.

Se no hipergrafo original temos:

Todos os vértices com grau limitado por um múltiplo de um certo $D$. 
Quase todos os vértices tem grau muito próximo a $D$.

A quantidade de arestas que contém dois vértices quaisquer é desprezível com respeito ao grau comum $D$.

Então obtemos

São escolhidas aproximadamente $\epsilon n / r$ hiperarestas. Estas arestas vão formar uma "cobertura parcial".

$\mathrm{O}$ número de vértices de $\mathcal{F}^{*}$ sofre um pequeno decrescimento exponencial $\mathrm{e}^{-\epsilon} \mathrm{com}$ respeito ao número de vértices do hipergrafo original $\mathcal{F}$.

O grau dos vértices de $\mathcal{F}^{*}$ decresce por um múltiplo deste fator linear no tamanho das hiperarestas com respeito ao grau dos vértices de $\mathcal{F}$.

As condições sobre o hipergrafo original $\mathcal{F}$ podem ser pensadas como condições de "alta regularidade" ou "altamente quase regularidade".

O problema é que o hipergrafo transformado $\mathcal{F}^{*}$ não precisa ser "tão regular quanto" $\mathcal{F}$. Essa perda de regularidade é indesejável pois no algoritmo que definiremos na continuação precisamos iterar essa transformação. Para contornar esse problema, permite-se que tanto as condições sobre $\mathcal{F}$ quanto aquelas sobre $\mathcal{F}^{*}$ sejam satisfeitas com uma certa "tolerância" a mais ou a menos. Essa tolerância ( $\delta$ no Lema 1.3.4) servirá como um parâmetro que nos permite "controlar" a perda de regularidade.

Algoritmo 1.3.5 Para provar a afirmativa do Teorema 1.3.3 itera-se a transformação descrita, criando em cada iteração um conjunto de arestas cuja união, depois da iteração final, formará a cobertura que garante a tese do teorema.

Porém a necessidade de controlar a perda das "condições de regularidade" requer tomar os seguintes cuidados com a tolerância e o limite para o grau usados na transformação.

Para determinar quais são os parâmetros $\delta, d$ e $n$ necessários para impor as condições (i), (ii), (iii) do Teorema 1.3.3) ao hipergrafo original $\mathcal{F}$ procedemos como segue.

Uma vez fixa a precisão com que pretendemos que $M(k, r, n)$ se ajuste a $\left(\begin{array}{l}k \\ r\end{array}\right)\left(\begin{array}{l}n \\ r\end{array}\right)^{-1}$, o controle que temos sobre a quantidade de hiperarestas escolhidas na transformação permite limitar o tamanho das coberturas (parciais) pelos termos de uma série geométrica de razão $\mathrm{e}^{-1}$. Isto nos permite determinar quantas iterações serão necessárias para cobrir uma quantidade grande de vértices.

Observe que em cada passo (iteração da transformação) cada cobertura parcial contribui com um fator exponencial para o tamanho da cobertura total procurada e, como devemos ajustar esse tamanho a $(n / r)(1+\epsilon)$, precisamos que a soma das parcelas seja controlada pelo $\epsilon$. Esse controle se executa usando as condições $(i v),(v),(v i)$ do Lema 1.3.4. Note que para tanto, precisamos escolher um outro $\epsilon^{\prime}$ pequeno o suficiente que o 
hipergrafo transformado satisfaça nossos requerimentos, i.e., para controlar os aportes à soma geométrica. Nessas condições o Lema 1.3.4 nos devolve o $\delta$ necessário para garantir as hipóteses sobre o hipergrafo original e aplicar com sucesso o Lema 1.3.4.

Esta estratégia define um processo de indução reversa finita no qual, em cada passo, estaremos determinando as condições precedentes que deve satisfazer o hipergrafo inicial. O processo termina quando atingimos o número de passos (determinados previamente) necessários para cobrir eficientemente as hiperarestas de $\mathcal{F}$.

Quando o processo termina, escolhemos $\delta$ para as hipóteses $(i),(i i),($ iii $)$ do Teorema 1.3.3 como o último $\delta$ gerado nas sucessivas aplicações do Lema 1.3.4. O número de vértices $n$ e o limitante para o grau $d$ serão escohidos, respectivamente, como os máximos entre todos os $n_{0}$ e $D_{0}$ gerados nas sucessivas aplicações do Lema 1.3.4. Todos essos cuidados serão descritos em mais detalhe na Seção 3.1 onde se expõe a prova do Teorema 1.3.3.

Antes de entrar em nossa matéria de estudo vamos fixar alguma notação.

Ao longo desta apresentação usaremos $\mathrm{P}$ para denotar probabilidades e E para o valor esperado de uma variável aleatória. Diz-se que uma variável aleatória $X$ tem distribuição binomial $B(n, p)$ com parâmetros $n$ e $p$ se $X$ conta o número de sucessos numa seqüência de $n$ experimentos independentes, onde cada sucesso tem a mesma probabilidade $p$ de ocorrência.

Vamos usar o símbolo $\sim$ em três contextos diferentes. Escrevemos $A \sim B$ querendo significar que o quociente $A / B$ tende a 1 quando $n$ (tipicamente o número de vértices de um hipergrafo) tende a infinito. Com $A \sim_{\epsilon} B$ denotaremos $1-\epsilon \leq A / B \leq 1+\epsilon$. Finalmente escrevemos " $A \sim B$ quase sempre" para denotar que, para todo $\epsilon>0$, a probabilidade $\mathrm{P}\left(A \sim_{\epsilon} B\right)$ tende a 1 quando $n$ tende a infinito; mais precisamente: Se $Z_{n}$ é uma variável aleatória indexada por $n$ e $z(n)$ é uma função de $n$ escrevemos

$$
\text { " } Z_{n} \sim z(n) \text { quase sempre" }
$$

se, para todo $\epsilon>0$, temos

$$
\lim _{n \rightarrow+\infty} \mathrm{P}\left(Z_{n} \sim_{\epsilon} z(n)\right)=1
$$

No decorrer do trabalho, $c_{1}, c_{2}, \ldots, c_{k}, \alpha, \beta, \gamma, \delta_{i}, \epsilon_{i}, \rho, \sigma$ denotam constantes apropriadas ao contexto. Se $X \subset V$ denotamos seu complemento por $\bar{X}=V \backslash X$. 


\section{Capítulo 2}

\section{Conjuntos Independentes}

\subsection{Grafos sem triângulos}

Consideremos o problema de determinar o número de independência de um grafo $G=$ ( $V G, E G)$, onde $V G$ denota o conjunto de vértices do grafo e $E G$ é o conjunto de aretas. Em um grafo qualquer, podemos proceder gulosamente, pondo em nosso conjunto independente, um vértice $x$ de grau mínimo e iterando este processo no grafo $G^{*}=G \backslash(\{x\} \cup \Gamma(x))$. Deste modo obtemos um conjunto independente que garante

$$
\alpha(G) \geq \frac{n}{t+1}=\Theta\left(\frac{n}{t}\right)
$$

onde $t$ é o grau médio do grafo.

Nessa seção vamos apresentar um resultado que melhora esse limite, para o caso de grafos sem triângulos, adicionando a ele um fator logarítmico em $t$. No que resta da seção assumiremos que $G$ é um grafo livre de triângulos. Consideraremos que o conjunto de vértices de grafo é $[n]=\{1, \ldots, n\}$ e que se $i \in[n]$ é um vértice, seu grau, denotado $d_{i}$, é o número de arestas incidentes ao vértice $i$. Aliás, o grau medio do grafo $G$, denotado $t=t(G)$, é definido por $t=\sum_{i=1}^{n} d_{i} / n=2|E G| / n$.

\subsubsection{A transformação}

Neste primeiro resultado a transformação mencionada na introdução vai significar o seguinte.

Achar um conjunto de vértices $C$ que induz um subgrafo $G[C]$ com número de independência alto (próximo à cardinalidade de $C$ ).

Definindo $V^{*}=V G \backslash(C \cup D)$, onde $D$ é o conjunto de vértices que são adjacentes a algum vértice de $C$, temos que no grafo $G^{*}=\left(V G^{*}, E G^{*}\right)$, induzido por $V^{*}$, o quociente entre o número de vértices e o grau médio não decresce demasiado em relação ao grafo original. 
Para provar estas afirmativas vamos escolher os vértices de $C$ ao acaso, pondo um vértice $x$ de $G$ em $C$ com probabilidade $p$ e deixando $x$ fora de $C$ com probabilidade $1-p$. Em seguida, demonstra-se que podemos escolher $p$ de modo que as condições que procuramos são satisfeitas com probabilidade não nula. A parte técnica da prova deste lema requer estimar $|E G[C]|,\left|V G^{*}\right|,\left|E G^{*}\right|$, usando a desigualdade de Chebyshev e a seguinte desigualdade exponencial análoga à desigualdade de Schwarz.

Lema 2.1.1 Seja $G=(V G, E G)$ um grafo sobre $n$ vértices e com grau máximo $\Delta$. Então

$$
\frac{1}{|E G|} \sum_{i j \in E G} \mathrm{e}^{-x\left(d_{i}+d_{j}\right)} \leq\left(\frac{1}{n} \sum_{i=1}^{n} \mathrm{e}^{-x d_{i}}\right)^{2}
$$

para todo $0 \leq x \leq \ln (1.25) / 4 \Delta$.

Demonstração. Embora comprida, a demostração deste lema é simples. A afirmativa seguinte esboça toda a estrategia da prova.

Afirmativa 2.1.2 Seja

$$
F(x)=\frac{1}{|E G|} \sum_{i j \in E G} \mathrm{e}^{-x\left(d_{i}+d_{j}\right)}-\frac{1}{n^{2}} \sum_{i, j=1}^{n} \mathrm{e}^{-x\left(d_{i}+d_{j}\right)} .
$$

Então

(i) $F(0)=0$,

(ii) $F^{\prime}(0) \leq 0$,

(iii) $F^{\prime}(x) \leq 0$ para todo $x \in[0, \ln (1.25) / 4 \Delta]$.

Estas três condições garantem que

$$
F(x) \leq 0, \text { para } 0 \leq x \leq 1 / 10 \Delta .
$$

É imediato que $(i)$ decorre da definição de $F(x)$; para verificar $(i i)$ e $(i i i)$ vamos fazer alguns cálculos.

\section{Afirmativa 2.1.3 Seja}

$$
H_{r}=\frac{1}{|E G|} \sum_{i j \in E G}\left(d_{i}+d_{j}-2 t\right)^{r}-\frac{1}{n^{2}} \sum_{i, j=1}^{n}\left(d_{i}+d_{j}-2 t\right)^{r} .
$$

Então

(iv) $H_{0}=0$, 
(v) $H_{1}=2 \sigma^{2} / t$, onde $\sigma^{2}$ é a variância de $d_{i}$,

(vi) $H_{l} \leq(2 \Delta)^{l-2}(4+4 \Delta / t) \sigma^{2}$ para todo $l \geq 2$.

Com efeito,

(iv)

$$
H_{0}=\frac{1}{|E G|} \sum_{i j \in E G} 1-\frac{1}{n^{2}} \sum_{i, j=1}^{n} 1=\frac{1}{|E G|}|E G|-\frac{1}{n^{2}} n^{2}=1-1=0 .
$$

(v) Vamos tratar em separado os termos de $H_{1}$.

$$
\begin{gathered}
\sum_{i j \in E G}\left(d_{i}+d_{j}-2 t\right)=\sum_{i j \in E G}\left(d_{i}+d_{j}\right)-2 t|E G| \\
=\sum_{i=1}^{n} d_{i}^{2}-t \sum_{i=1}^{n} d_{i}=\sum_{i=1}^{n} d_{i}\left(d_{i}-t\right) .
\end{gathered}
$$

Para o segundo termo temos

$$
\begin{gathered}
\sum_{i, j=1}^{n}\left(d_{i}+d_{j}-2 t\right)=\sum_{i, j=1}^{n}\left(d_{i}+d_{j}\right)-2 t n^{2} \\
=2 n\left(\sum_{i=1}^{n} d_{i}\right)-2 t n^{2}=2 n(2|E G|)-2 t n^{2}=0 .
\end{gathered}
$$

Portanto,

$$
H_{1}=\frac{2}{n t} \sum_{i=1}^{n} d_{i}\left(d_{i}-t\right)=\frac{2}{t}\left(\left(\frac{1}{n} \sum_{i=1}^{n} d_{i}^{2}\right)-t^{2}\right) .
$$

Observemos que a última expressão entre parênteses $\sigma^{2}=\left(\frac{1}{n} \sum_{i=1}^{n} d_{i}^{2}\right)-t^{2}$ é a variância do grau $d_{i}$ de um vértice $i$.

(vi) Desconsiderando o segundo termo de $H_{l}$ temos

$$
\begin{aligned}
H_{l} & \leq \frac{2}{n t} \sum_{i j \in E G}\left(d_{i}+d_{j}-2 t\right)(2 \Delta)^{l-1} \\
& =(2 \Delta)^{l-1} \frac{2}{n t} \sum_{i=1}^{n} d_{i}\left(d_{i}-t\right) \\
= & (2 \Delta)^{l-1} \frac{2 \sigma^{2}}{t}=(2 \Delta)^{l-2} \frac{4 \Delta}{t} \sigma^{2}
\end{aligned}
$$

e isto basta para garantir (vi). 


\section{Afirmativa 2.1.4 Seja}

$$
G_{r}=\frac{1}{|E G|} \sum_{i j \in E G}\left(d_{i}+d_{j}\right)^{r}-\frac{1}{n^{2}} \sum_{i, j=1}^{n}\left(d_{i}+d_{j}\right)^{r} .
$$

\section{Então}

(vii) $G_{r}=\sum_{l=0}^{r}\left(\begin{array}{l}r \\ l\end{array}\right) H_{l}(2 t)^{r-l}$ para $r \geq 0$.

(viii) $G_{0}=0$,

(ix) $G_{1} \geq 0$,

(x) $G_{r}<(4 \Delta)^{r} 2 \sigma^{2} / \Delta t$ para todo $r \geq 2$.

Com efeito,

(vii)

$$
G_{r}=\frac{1}{|E G|} \sum_{i j \in E G}\left(d_{i}+d_{j}-2 t+2 t\right)^{r}-\frac{1}{n^{2}} \sum_{i, j=1}^{n}\left(d_{i}+d_{j}-2 t+2 t\right)^{r}
$$

e pelo teorema do binômio

$$
\begin{gathered}
=\frac{1}{|E G|} \sum_{i j \in E G} \sum_{l=0}^{r}\left(\begin{array}{l}
r \\
l
\end{array}\right)\left(d_{i}+d_{j}-2 t\right)^{l}(2 t)^{r-l}-\frac{1}{n^{2}} \sum_{i, j=1}^{n} \sum_{l=0}^{r}\left(\begin{array}{l}
r \\
l
\end{array}\right)\left(d_{i}+d_{j}-2 t\right)^{l}(2 t)^{r-l} \\
=\sum_{l=0}^{r}\left(\begin{array}{l}
r \\
l
\end{array}\right)\left\{\frac{1}{|E G|} \sum_{i j \in E G}\left(d_{i}+d_{j}-2 t\right)^{l}-\frac{1}{n^{2}} \sum_{i, j=1}^{n}\left(d_{i}+d_{j}-2 t\right)^{l}\right\}(2 t)^{r-l} .
\end{gathered}
$$

(viii) Decorre imediatamente de $(i v)$.

(ix) De (vii), $(i v)$ e $(v)$ temos

$$
G_{1}=\sum_{l=0}^{1}\left(\begin{array}{l}
1 \\
l
\end{array}\right) H_{l}(2 t)^{1-l}=\left(\begin{array}{l}
1 \\
0
\end{array}\right) H_{0}(2 t)+\left(\begin{array}{l}
1 \\
1
\end{array}\right) H_{1}=H_{1}=2 \sigma^{2} / t .
$$

e portanto $G_{1}>0$ pois $\sigma^{2} \geq 0$ ao ser uma variância, como já dizemos, no final da prova de $(v)$. Outro jeito de ver isso é usando a desigualdade de Schwarz. Consideremos os vetores $n$-dimensionais $x=\left(d_{1}, \ldots, d_{n}\right)$ e $y=(1, \ldots, 1)$; pela desigualdade de Schwarz temos

$$
\left(\sum_{i=1}^{n} d_{i}\right)^{2} \leq n \sum_{i=1}^{n} d_{i}^{2}
$$

ou seja

$$
n t^{2} \leq \sum_{i=1}^{n} d_{i}^{2}
$$


Observamos que $G_{1}=0$ quando $\sigma^{2}=0$, isto é, quando o grafo é regular (pelo resultado em $\mathbb{R}: x=t y)$.

(x) De (vii) e (vi) temos

$$
\begin{gathered}
G_{r}=\sum_{l=0}^{r}\left(\begin{array}{l}
r \\
l
\end{array}\right) H_{l}(2 t)^{r-l} \leq \sum_{l=0}^{r}\left(\begin{array}{l}
r \\
l
\end{array}\right)(2 \Delta)^{l-2}(4+4 \Delta / t) \sigma^{2}(2 t)^{r-l} \\
=(4+4 \Delta / t)(2 \Delta)^{-2} \sigma^{2}\left(\sum_{l=0}^{r}\left(\begin{array}{l}
r \\
l
\end{array}\right)(2 \Delta)^{l}(2 t)^{r-l}\right)=(4+4 \Delta / t)(2 \Delta)^{-2}(2 \Delta+2 t)^{r} \sigma^{2}
\end{gathered}
$$

e portanto

$$
G_{r} \leq\left(\frac{t+\Delta}{\Delta}\right) \frac{1}{\Delta t}(2 \Delta+2 t)^{r} \sigma^{2}
$$

Para provar $(i i)$ e $(i i i)$ expressamos $F(x)$ segundo a fórmula de Taylor

$$
F(x)=\sum_{r=0}^{\infty} F^{(r)}(0) \frac{x^{r}}{r !}
$$

Segue-se por indução que

$$
F^{(r)}(x)=\frac{d^{r}}{d x}(F(x))=(-1)^{r}\left\{\frac{1}{|E G|} \sum_{i j \in E G}\left(d_{i}+d_{j}\right)^{r} \mathrm{e}^{-x\left(d_{i}+d_{j}\right)}-\frac{1}{n^{2}} \sum_{i, j=1}^{n}\left(d_{i}+d_{j}\right)^{r} \mathrm{e}^{-x\left(d_{i}+d_{j}\right)}\right\}
$$

e portanto

$$
F^{(r)}(0)=(-1)^{r} G_{r}, \text { para todo } r \geq 0 .
$$

Temos assim que $(i x)$ implica $F^{\prime}(0) \leq 0$, garantindo $(i i)$.

Além disso

$$
F^{\prime}(x)=\sum_{r=0}^{\infty} F^{(r+1)}(0) \frac{x^{r}}{r !}=F^{\prime}(0)+\sum_{r=1}^{\infty} F^{(r+1)}(0) \frac{x^{r}}{r !} .
$$

De $(x)$ temos

$$
\begin{gathered}
\left|F^{(r+1)}(0)\right| x^{r}=G_{r+1} x^{r} \leq(4 \Delta)^{r+1} \frac{2 \sigma^{2}}{\Delta t} x^{r} \\
\sum_{r=1}^{\infty}\left|F^{(r+1)}(0)\right| \frac{x^{r}}{r !}=\sum_{r=1}^{\infty}\left|G_{r+1}\right| \frac{x^{r}}{r !} \leq \sum_{r=1}^{\infty}(4 \Delta)^{r+1} \frac{2 \sigma^{2}}{\Delta t} \frac{x^{r}}{r !} \\
=\frac{2 \sigma^{2}}{t} 4 \sum_{r=1}^{\infty} \frac{(4 \Delta x)^{r}}{r !}=\frac{2 \sigma^{2}}{t} 4\left\{\sum_{r=0}^{\infty} \frac{(4 \Delta x)^{r}}{r !}-1\right\}=\frac{2 \sigma^{2}}{t} 4\left(\mathrm{e}^{4 \Delta x}-1\right)
\end{gathered}
$$

e portanto para satisfazer (iii) basta que

$$
4\left(\mathrm{e}^{4 \Delta x}-1\right)<1
$$

o qual é válido para todo $x \in[0, \ln (1.25) / 4 \Delta]$; observe que a série converge nesse intervalo. 
Lema 2.1.5 (A transformação) Seja $G=(V G, E G)$ um grafo sobre $n$ vértices e consideremos um conjunto $C \subset V G$. Definimos

$$
\begin{aligned}
D & =\{x \in V G: \exists y \in C \text { tal que } x y \in E G\}=\Gamma(C)=\bigcup_{x \in C} \Gamma(x), \\
I & =C \cap \bar{D}=C \backslash D=C \backslash \Gamma(C), \\
V^{*} & =\bar{C} \cap \bar{D}=V \backslash(C \cup D)=V \backslash(C \cup \Gamma(C)) .
\end{aligned}
$$

Então o subgrafo $G^{*}=\left(V G^{*}, E G^{*}\right)$ de $G$, induzido por $V^{*}$, satisfaz

$$
\alpha(G) \geq \alpha(G[C])+\alpha\left(G^{*}\right) \geq|I|+\alpha\left(G^{*}\right)
$$

ou seja, I é um conjunto independente de $G$ que não contém vértices de $G^{*}$.

Com efeito, pela sua definição, $I$ não induz arestas em $G$ e aliás $V^{*} \cap I=\emptyset$. Note também que não pode haver arestas ligando um vértice de $I$ a um vértice de $V^{*}$. 
Lema 2.1.6 (Ajtai, Komlós e Szemerédi, '81; [2]) Seja $G=(V G, E G)$ um grafo sobre $n$ vértices, sem triângulos, com grau médio $t$ e grau máximo $\Delta$. Então existe um conjunto $C \subset V G$, satisfazendo

(i) $|C|>n /(100 t)$,

(ii) $|E G[C]|<|C| / 50$

e tal que o subgrafo $G^{*}=\left(V G^{*}, E G^{*}\right)$ de $G$, induzido por $V^{*}$ segundo o Lema 2.1.5, sobre $n^{\prime}$ vértices e com grau médio $t^{\prime}$, satisfaz

(iii) $\left|V G^{*}\right|>n / 2$,

(iv) $n^{\prime} / t^{\prime}=\left|V G^{*}\right|^{2} /\left(2\left|E G^{*}\right|\right)>\vartheta|V G|^{2} /(2|E G|)=\vartheta n / t$.

onde $\vartheta=1-1 / t-c_{3} \sqrt{t / n}$.

Demonstração. Assumimos primeiro que $10 t=\Delta$ e escolhemos um conjunto $C \subset V$ de cardinalidade $k=n /(100 t)$. Dado $C$, consideramos $G^{*}=\left(V G^{*}, E G^{*}\right)$, o grafo induzido pela transformação $(2.1 .5)$.

Estimação de $E G[C]$. Definimos

$$
X_{i j}(C)= \begin{cases}1 & \text { se } i \in C \text { e } j \in C, \\ 0 & \text { se } i \notin C \text { ou } j \notin C .\end{cases}
$$

Para $i j \in E G$ fixo e arbitrário temos,

$$
\begin{aligned}
\mathrm{E}\left(X_{i j}(C)\right) & =\sum \mathrm{P}\left(X_{i j}(C)=1\right), \quad \text { onde a soma é indizapa por } C \subset V G:|C|=k \\
& =\sum \mathrm{P}(i \in C \text { e } j \in C), \quad \text { onde a soma é indizada por } C \subset V G:|C|=k \\
& =\left(\begin{array}{l}
n-2 \\
k-2
\end{array}\right) /\left(\begin{array}{l}
n \\
k
\end{array}\right) .
\end{aligned}
$$

Portanto

$$
\begin{gathered}
\mathrm{E}(|E G[C]|)=\sum_{i j \in E G} \mathrm{E}\left(X_{i j}(C)\right)=|E G|\left(\begin{array}{l}
n-2 \\
k-2
\end{array}\right) /\left(\begin{array}{l}
n \\
k
\end{array}\right) \\
\mathrm{E}(|E G[C]|)=\frac{k(k-1) t}{2(n-1)} \sim \frac{k^{2} t}{2 n} \\
\operatorname{Var}(|E G[C]|)=\sum_{i j \in E G, r s \in E G} \mathrm{E}\left(X_{i j}(C) X_{r s}(C)\right)-\mathrm{E}\left(X_{i j}(C)\right) \mathrm{E}\left(X_{r s}(C)\right) \\
=\sum_{i j \in E G, r s \in E G}\left\{\left(\begin{array}{l}
n-g(i j, r s) \\
k-g(i j, r s)
\end{array}\right) /\left(\begin{array}{l}
n \\
k
\end{array}\right)-\frac{k^{2}(k-1)^{2}}{n^{2}(n-1)^{2}}\right\}
\end{gathered}
$$


onde $g(i j, r s)$ é o número de vértices determinado pelas arestas $i j$ e $r s$.

$$
=\sum_{i j \in E G} \operatorname{Var}\left(X_{i j}(C)\right)+\sum\left\{\left(\begin{array}{l}
n-3 \\
k-3
\end{array}\right) /\left(\begin{array}{l}
n \\
k
\end{array}\right)-\frac{k^{2}(k-1)^{2}}{n^{2}(n-1)^{2}}\right\}
$$

onde a segunda soma é indizada por $i j, r s \in E G: g(i j, r s)=3$. Assim, o primeiro somando corresponde aos pares de arestas determinados por dois vértices e o segundo somando corresponde aos pares de arestas que são definidas por três vértices; observe que se $g(i j, r s)=4$ então $\operatorname{Cov}\left(X_{i j}(C), X_{r s}(C)\right)=0$.

Já que a variância pode-se limitar pela esperança e que a quantidade de pares de $\operatorname{arestas}(i j, r s)$ para as quais $g(i j, r s)=3$ é limitada superiormente por $\sum_{i=1}^{n} t_{i}^{2}<n \Delta^{2}$ temos

$$
\operatorname{Var}(|E G[C]|)<k^{2} \frac{t}{n}+n \Delta^{2} \frac{k^{3}}{n^{3}}=k^{2} \frac{t}{n}+k^{3} \frac{t^{2}}{n^{2}}
$$

portanto

$$
\operatorname{Var}(|E G[C]|)<2 k^{3} \frac{t^{2}}{n^{2}}
$$

Assim

$$
\sqrt{\operatorname{Var}(|E G[C]|)} / \mathrm{E}(|E G[C]|)<400 \sqrt{t / n} .
$$

Estimação de $\left|V G^{*}\right|$. Definimos

$$
X_{i}^{*}(C)= \begin{cases}1 & \text { se }\left(\Gamma_{i} \cup\{i\}\right) \cap C=\emptyset \\ 0 & \text { caso contrário. }\end{cases}
$$

Para $i \in V G$ fixo e arbitrário temos,

$$
\begin{aligned}
\mathrm{E}\left(X_{i}^{*}(C)\right) & =\sum_{C \subset V G:|C|=k} \mathrm{P}\left(X_{i}^{*}(C)=1\right) \\
& =\sum \mathrm{P}\left(\left(\Gamma_{i} \cup\{i\}\right) \cap C=\emptyset\right) \quad \text { onde a soma é indizada por } C \subset V G:|C|=k \\
& =\left(\begin{array}{c}
n-d_{i}-1 \\
k
\end{array}\right) /\left(\begin{array}{l}
n \\
k
\end{array}\right)
\end{aligned}
$$

portanto

$$
\mathrm{E}\left(\left|V G^{*}\right|\right)=\sum_{i=1}^{n} \mathrm{E}\left(X_{i}^{*}(C)\right)=\sum_{i=1}^{n}\left(\begin{array}{c}
n-d_{i}-1 \\
k
\end{array}\right) /\left(\begin{array}{l}
n \\
k
\end{array}\right)
$$

pela convexidade de $f(x)=\left(\begin{array}{l}x \\ k\end{array}\right)$ temos

$$
\mathrm{E}\left(\left|V G^{*}\right|\right) \geq n\left(\begin{array}{c}
\sum_{i=1}^{n} \frac{n-d_{i}-1}{n} \\
k
\end{array}\right) /\left(\begin{array}{l}
n \\
k
\end{array}\right)=n\left(\begin{array}{c}
n-t-1 \\
k
\end{array}\right) /\left(\begin{array}{l}
n \\
k
\end{array}\right)
$$




$$
=n \frac{(n-t-1)_{k}}{(n)_{k}}>n \frac{(n-t-1)^{k}}{n^{k}}\left(1-\frac{k}{n-t}\right)^{k} .
$$

Portanto

$$
\mathrm{E}\left(\left|V G^{*}\right|\right)>0.9 n
$$

Agora limitamos superiormente a variância.

$$
\begin{aligned}
& \operatorname{Var}\left(\left|V G^{*}\right|\right)=\sum_{i, j=1}^{n}\left\{\mathrm{E}\left(X_{i}^{*}(C) X_{j}^{*}(C)\right)-\mathrm{E}\left(X_{i}^{*}(C)\right) \mathrm{E}\left(X_{j}^{*}(C)\right)\right\} \\
& =\sum_{i, j=1}^{n}\left\{\left(\begin{array}{c}
n-N_{i j} \\
k
\end{array}\right) /\left(\begin{array}{l}
n \\
k
\end{array}\right)-\left(\begin{array}{c}
n-N_{i} \\
k
\end{array}\right)\left(\begin{array}{c}
n-N_{j} \\
k
\end{array}\right) /\left(\begin{array}{l}
n \\
k
\end{array}\right)^{2}\right\}
\end{aligned}
$$

onde $N_{i}=d_{i}+1, N_{j}=d_{j}+1$ e $N_{i j}=\left|\Gamma_{i} \cup\{i\} \cup \Gamma_{j} \cup\{j\}\right|$. Para estimar a variância, sustraímos e adicionamos aos termos desta soma a quantidade

$$
\left(\begin{array}{c}
n-N_{i}-N_{j} \\
k
\end{array}\right) /\left(\begin{array}{l}
n \\
k
\end{array}\right)
$$

e já que $(n)_{k}\left(n-N_{1}-N_{2}\right)_{k} \leq\left(n-N_{1}\right)_{k}\left(n-N_{2}\right)_{k}$ podemos descartar as diferenças negativas criadas em cada termo, e temos

$$
\begin{gathered}
\operatorname{Var}\left(\left|V G^{*}\right|\right) \leq \sum_{i, j=1}^{n}\left\{\left(\begin{array}{c}
n-N_{i j} \\
k
\end{array}\right)-\left(\begin{array}{c}
n-N_{i}-N_{j} \\
k
\end{array}\right)\right\} /\left(\begin{array}{l}
n \\
k
\end{array}\right) \\
\leq \sum_{i, j=1}^{n} \sum_{h=N_{i j}+1}^{N_{i}+N_{j}}\left(\begin{array}{c}
n-h \\
k-1
\end{array}\right) /\left(\begin{array}{l}
n \\
k
\end{array}\right) \\
=\frac{k}{n} \sum_{i, j=1}^{n} \sum_{h=N_{i j}+1}^{N_{i}+N_{j}}\left(\frac{n-h}{n-1}\right)^{k} \leq \frac{k}{n} \sum_{i, j=1}^{n}\left(N_{i}+N_{j}-N_{i j}\right) \\
=\frac{k}{n} \sum_{i=1}^{n}\left\{\sum_{j=1: j=i}^{n}\left(N_{i}+N_{j}-N_{i j}\right)+\sum_{j \in V G: i j \in E G}\left(N_{i}+N_{j}-N_{i j}\right)+\sum_{j \in V G: i j \notin E G}\left(N_{i}+N_{j}-N_{i j}\right)\right\} \\
=\frac{k}{n} \sum_{i=1}^{n}\left\{\left(d_{i}+1\right)+\left(2 d_{i}\right)+\sum_{j=1: i j \notin E G}^{n}\left|\Gamma_{i} \cup \Gamma_{j}\right|\right\}=\frac{k}{n} \sum_{i=1}^{n}\left\{\left(d_{i}+1\right)+\left(2 d_{i}\right)+d_{i}\left(d_{i}-1\right)\right\} \\
=\frac{k}{n} \sum_{i=1}^{n}\left(d_{i}+1\right)^{2} \leq \frac{k}{n} \sum_{i=1}^{n}\left(d_{i}+1\right)(\Delta+1) \leq \frac{k}{n} n(t+1)(\Delta+1) \\
=\frac{n(t+1)}{10}\left(\frac{10 t+1}{10 t}\right)<\frac{n(t+1)}{5}<n t
\end{gathered}
$$

portanto

$$
\operatorname{Var}\left(\left|V G^{*}\right|\right)<n t
$$


e assim

$$
\sqrt{\operatorname{Var}\left(\left|V G^{*}\right|\right)} / \mathrm{E}\left(\left|V G^{*}\right|\right)<2 \sqrt{t / n}
$$

Estimação de $\left|E G^{*}\right|$. Definimos, para cada aresta $i j \in E G$,

$$
X_{i j}^{*}(C)= \begin{cases}1 & \text { se }\left(\Gamma_{i} \cup\{i\} \cup \Gamma_{j} \cup\{j\}\right) \cap C=\emptyset, \\ 0 & \text { caso contrário. }\end{cases}
$$

Para $i j \in E G$ fixa e arbitrária temos,

$$
\begin{gathered}
\mathrm{E}\left(X_{i j}^{*}(C)\right)=\sum_{C \subset V G:|C|=k} \mathrm{P}\left(X_{i j}^{*}(C)=1\right) \\
=\sum_{C \subset V G:|C|=k} \mathrm{P}\left(\left(\Gamma_{i} \cup\{i\} \cup \Gamma_{j} \cup\{j\}\right) \cap C=\emptyset\right)=\left(\begin{array}{c}
n-N_{i j} \\
k
\end{array}\right) /\left(\begin{array}{l}
n \\
k
\end{array}\right)
\end{gathered}
$$

portanto

$$
\mathrm{E}\left(\left|E G^{*}\right|\right)=\sum_{i j \in E G} \mathrm{E}\left(X_{i j}^{*}(C)\right)=\sum_{i j \in E G}\left(\begin{array}{c}
n-N_{i j} \\
k
\end{array}\right) /\left(\begin{array}{l}
n \\
k
\end{array}\right)
$$

pela convexidade de $f(x)=\left(\begin{array}{l}x \\ k\end{array}\right)$ temos

$$
\geq \frac{n t}{2}\left(\begin{array}{c}
\sum_{i j \in E G}\left(n-N_{i j}\right) /|E G| \\
k
\end{array}\right) /\left(\begin{array}{l}
n \\
k
\end{array}\right)
$$

já que o grafo não tem triângulos

$$
=\frac{n t}{2}\left(\begin{array}{c}
n-\sum_{i=1}^{n} d_{i}^{2} /|E G| \\
k
\end{array}\right) /\left(\begin{array}{l}
n \\
k
\end{array}\right)
$$

e como $f(x)=\left(\begin{array}{l}x \\ k\end{array}\right)$ é uma função crescente, para todo $x \geq k$, temos

$$
\begin{gathered}
\geq \frac{n t}{2}\left(\begin{array}{c}
n-2 \Delta-1 \\
k
\end{array}\right) /\left(\begin{array}{l}
n \\
k
\end{array}\right)=\frac{n t}{2} \frac{(n-2 \Delta-1)_{k}}{(n)_{k}} \\
\geq \frac{n t}{2} \frac{(n-2 \Delta)^{k}}{(n)_{k}}\left(1-\frac{k}{n-2 \Delta}\right)^{k}>\frac{n t}{2}\left(1-\frac{k}{n-2 \Delta}\right)^{k}>n t / 10
\end{gathered}
$$

portanto

$$
\mathrm{E}\left(\left|E G^{*}\right|\right)>0.1 n t
$$

Agora limitamos superiormente a variância.

$$
\operatorname{Var}\left(\left|E G^{*}\right|\right)=\sum_{i j, r s \in E G}\left\{\left(\begin{array}{c}
n-N_{i j, r s} \\
k
\end{array}\right) /\left(\begin{array}{l}
n \\
k
\end{array}\right)-\left(\begin{array}{c}
n-N_{i j} \\
k
\end{array}\right)\left(\begin{array}{c}
n-N_{r s} \\
k
\end{array}\right) /\left(\begin{array}{l}
n \\
k
\end{array}\right)^{2}\right\}
$$


onde $N_{i j, r s}=\left|\Gamma_{i} \cup\{i\} \cup \Gamma_{j} \cup\{j\} \cup \Gamma_{r} \cup\{r\} \cup \Gamma_{s} \cup\{s\}\right|$. Sustraindo e adicionando aos termos dessa soma a quantidade

$$
\left(\begin{array}{c}
n-N_{i j}-N_{r s} \\
k
\end{array}\right) /\left(\begin{array}{l}
n \\
k
\end{array}\right)
$$

e depois de descartar as diferenças negativas criadas em cada termo, temos

$$
\begin{gathered}
\operatorname{Var}\left(\left|E G^{*}\right|\right) \leq \sum_{i j, r s \in E G}\left\{\left(\begin{array}{c}
n-N_{i j, r s} \\
k
\end{array}\right)-\left(\begin{array}{c}
n-N_{i j}-N_{r s} \\
k
\end{array}\right)\right\} /\left(\begin{array}{l}
n \\
k
\end{array}\right) \\
\leq \sum_{i j, r s \in E G} \sum_{h=N_{i j, r s}+1}^{N_{i j}+N_{r s}}\left(\begin{array}{c}
n-h \\
k
\end{array}\right) /\left(\begin{array}{l}
n \\
k
\end{array}\right) \\
\leq \sum_{i j, r s \in E G} \sum_{h=N_{i j, r s}+1}^{N_{i j}+N_{r s}} \frac{k}{n}\left(\frac{n-h}{n-1}\right)^{k} \\
\leq \frac{k}{n} \sum_{i j, r s \in E G}\left(N_{i j}+N_{r s}-N_{i j, r s}\right)=\frac{k}{n} \sum_{i j, r s \in E G}\left|N_{i j} \cap N_{r s}\right| \\
=\frac{k}{n} \sum_{i j, r s \in E G} \sum_{x \in\left|N_{i j} \cap N_{r s}\right|} 1=\frac{k}{n} \sum_{x \in V} \sum_{i j, r s \in E G: x \in\left|N_{i j} \cap N_{r s}\right|} 1 \\
\leq \frac{k}{n} \sum_{x \in V} \sum_{u, v \in \Gamma_{x}} d_{u} d_{v} \leq \frac{k}{n} \sum_{x \in V} \Delta^{4}=\frac{k}{n} n \Delta^{4}
\end{gathered}
$$

portanto

$$
\operatorname{Var}\left(\left|E G^{*}\right|\right)<100 n t^{3}
$$

e assim

$$
\sqrt{\operatorname{Var}\left(\left|E G^{*}\right|\right)} / \mathrm{E}\left(\left|E G^{*}\right|\right)<100 \sqrt{t / n} .
$$

As desigualdades (2.2), (2.3), e (2.4), e a desigualdade de Chebyshev garantem que existe um conjunto $C \subset V$ tal que para $\delta$ suficientemente pequeno valem

$$
\begin{aligned}
\left|V G^{*}\right| & >\mathrm{E}\left(\left|V G^{*}\right|\right)(1-\delta), \\
\left|E G^{*}\right| & <\mathrm{E}\left(\left|E G^{*}\right|\right)(1+\delta), \\
|E G[C]| & <\mathrm{E}(|E G[C]|)(1+\delta) .
\end{aligned}
$$


Das contas anteriores, tomando $\delta=O(\sqrt{t / n})$, temos

$$
\begin{aligned}
\left(\mathrm{E}\left|V G^{*}\right|\right)^{2} /\left(2 \mathrm{E}\left(\left|E G^{*}\right|\right)\right) & =\frac{\left(\sum_{i=1}^{n}\left(\begin{array}{c}
n-N_{i} \\
k
\end{array}\right) /\left(\begin{array}{l}
n \\
k
\end{array}\right)\right)^{2}}{2 \sum_{i j \in E G}\left(\begin{array}{c}
n-N_{i j} \\
k
\end{array}\right) /\left(\begin{array}{l}
n \\
k
\end{array}\right)} \\
& \sim \frac{\left(\sum_{i=1}^{n} \frac{(n)^{k}}{(n)_{k}} \mathrm{e}^{-(1+o(1)) \frac{d_{i} k}{n}-(1+o(1)) \frac{k}{n}}\right)^{2}}{2 \sum_{i j \in E G} \frac{(n)^{k}}{(n-k+1)_{k}} \mathrm{e}^{-(1+o(1))\left(d_{i}+d_{j}\right) \frac{k}{n}}} \\
& >\frac{n^{2}}{2|E G|} \frac{\left(\frac{1}{n} \sum_{i=1}^{n} \mathrm{e}^{-k d_{i} / n}\right)^{2}}{|E G|} \mathrm{e}_{i j \in E G} \mathrm{e}^{-k\left(d_{i}+d_{j}\right) / n} \mathrm{e}^{-1 / 100 t} \\
& >\left(1-\frac{1}{t}\right) \frac{n}{t}
\end{aligned}
$$

sendo que a penúltima desigualdade é devida ao Lema 2.1.1. Então (2.5) e (2.6) garantem que

$$
\left|V G^{*}\right|^{2} /\left(2\left|E G^{*}\right|\right)>\frac{(1-\delta)^{2}}{(1+\delta)}\left(1-\frac{1}{t}\right) \frac{n}{t}>\vartheta \frac{n}{t}
$$

também

$$
\left|V G^{*}\right|>(1-\delta) \mathrm{E}\left(\left|V G^{*}\right|\right)>\vartheta 0.9 n
$$

A afirmativa (ii) decorre de 2.1 e 2.7.

No caso em que o grafo não satisfaz max $d_{i} \leq 10 t$, apagamos todos os vértices de grau $\geq 10 t$ e aplicamos a construção descrita ao grafo sobre os $n^{\prime}$ vértices restantes. Neste caso, a desigualdade de Markov mostra que $\mathrm{P}\left(d_{i} \geq 10 t\right) \leq 0.1$ e, portanto, temos $n^{\prime} \geq 0.9 n$; segue-se que $n^{\prime} / t^{\prime} \geq 0.9 n / t$; o limite inferior para $\left|V G^{*}\right|$ muda para

$$
\left|V G^{*}\right|>\vartheta(0.9)^{2} n>n / 2
$$

e os outros não são afetados. 


\subsubsection{O algoritmo}

Sempre temos que o número de independência de um grafo $G$ sobre $n$ vértices e grau médio $t$ e maior o igual a $n / t$. Em nosso caso, para construir efetivamente um tal conjunto independente, sorteamos um conjunto $C$ como descrito no Lema 2.1.6, tantas vezes como seja necessário até conseguirmos algum que satisfaça as afirmativas deste lema. A afirmativa (ii) garante que, no máximo, $2|C| / 50$ dos vértices são cobertos por arestas do grafo induzido por $C$; portanto, temos que $C$ contém um conjunto independente de vértices de tamanho, pelo menos, $0.01 n / t$.

Vamos construir um conjunto independente "grande" em $G$ iterando o processo descrito no Lema 2.1.6 um número $\Theta(\log t)$ de vezes, escolhendo cada vez os grafos induzidos $G[C]$ e $G^{*}$ no grafo transformado $G$ da iteração anterior. Isso fornece em cada iteração um conjunto independente grande, a união dos quais vai garantir a afirmativa do lema.

Teorema 2.1.7 (Ajtai, Komlós e Szemerédi, '81; [2]) Se um grafo livre de triângulos $G$ com $n$ vértices tem grau médio $t>c_{1}$, então

$$
\alpha(G)>c_{4} n(\log t) / t
$$

Demonstração. Já que $G$ não tem triângulos, segue que $\alpha(G) \geq t$ e, como sempre temos $n>t$, segue que se $t \geq \sqrt{n \log n}$, teremos

$$
n>t>\sqrt{n \log n}
$$

implica

$$
\frac{\sqrt{n}}{t}>\frac{1}{\sqrt{n}}>\frac{\sqrt{\log n}}{t}
$$

e portanto

$$
\sqrt{n \log n}=n \frac{1}{\sqrt{n}} \sqrt{\log n}>n \frac{\log n}{t}>n \frac{\log t}{t}
$$

e temos a tese.

Para o caso $t<\sqrt{n \log n}$, consideremos o seguinte algoritmo. 
Algoritmo 2.1.8 Entrada: Um grafo $G=(V G, E G)$ sobre $n$ vértices, sem triângulos e com grau médio t.

Saída: Um conjunto independente $I$ de tamanho $\geq c(n / t)(\log t)$.

1.Ponha $G_{0}=G, t_{0}=t, \vartheta_{0}=1, r=1$.

2. Enquanto $r<(\log t) / 2$ e $\vartheta_{r}>1-1 /(\log t)$

Sorteie um conjunto C como descrito no Lema 2.1.6 e verifique se esse conjunto satisfaz as afirmativas do lema; caso contrário, volte a sortear até conseguir um conjunto $C$ adequado.

Ponha

$$
\begin{aligned}
& G_{r}=G_{r-1}^{*}, \\
& I_{r} \text { o conjunto independente de } V G_{r-1}[C], \\
& n_{r}=\left|V G_{r}\right|, \\
& t_{r}=t\left(G_{r}\right) e \\
& \vartheta_{r}=1-1 / t_{r}-c_{3} \sqrt{t_{r} / n_{r}} .
\end{aligned}
$$

3. Devolva $I=\bigcup_{s=0}^{r} I_{s}$.

Lema 2.1.9 (Invariantes) Em cada passo "s" da execução do algoritmo (2.1.8) valem

(i) $n_{s}>n / 2^{s}$,

(ii) $n_{s} / t_{s}>(1-1 /(\log t))^{s} n / t$.

Demonstração. As afirmativas provam-se por indução matemática.

(i) Caso base : (iii) do Lema 2.1.6 garante que $n_{1}>n_{0} / 2$.

Passo indutivo : Seja $s>1$ e suponhamos que $(i)$ vale para $s-1$. Então, novamente por (iii) do Lema 2.1.6, temos $n_{s}>n_{s-1} / 2$ e, pela hipótese indutiva, $n_{s}>n / 2 \times 2^{s-1}=$ $n / 2^{s}$.

(ii) Caso base : (iv) do Lema 2.1.6 e a definição do laço do Algoritmo 2.1.8 garantem que $n_{1} / t_{1}>(1-1 /(\log t)) n / t$

Passo indutivo : Seja $s>1$ e suponhamos que $(i i)$ vale para $s-1$. Então por $(i v)$ do Lema 2.1.6 temos $n_{s} / t_{s}>\vartheta n_{s-1} / t_{s-1}$, pela hipótese indutiva temos $n_{s} / t_{s}>\vartheta(1-$ $1 /(\log t))^{s-1} n / t$ e a definição do laço do Algoritmo 2.1 .8 garante a tese. 
Lema 2.1.10 (Certificado) O conjunto I devolvido pelo Algoritmo 2.1.8 é um conjunto independente do grafo $G$ e satisfaz

$$
|I| \geq c_{4} n(\log t) / t .
$$

Demonstração. Pela definição do Algoritmo 2.1.8, temos que considerar os seguintes casos.

1. Suponhamos que a execução do algoritmo termina e $\vartheta_{r}>1-1 /(\log t)$. Portanto $r=(\log t) / 2 ;$ temos,

$$
|I|=\sum_{i=0}^{r}\left|I_{s}\right|>\sum_{s=1}^{r} \frac{n_{s}}{t_{s}} .
$$

Então, usando o invariante (ii) do lema anterior, obtemos

$$
\begin{aligned}
|I| & >\frac{n}{t} \sum_{s=1}^{r}(1-1 / \log t)^{s} \\
& =\frac{n}{t} \sum_{s=1}^{(\log t) / 2}(1-1 / \log t)^{s} \\
& =\frac{n}{t}\left\{\frac{1-(1-1 / \log t)^{(\log t) / 2}}{1-(1-1 / \log t)}\right\} \\
& =\frac{n}{t} \frac{\left(1-\mathrm{e}^{-1 / 2}\right)}{(1-1+1 / \log t)} \\
& =\left(1-\mathrm{e}^{-1 / 2}\right) \frac{n}{t}(\log t)
\end{aligned}
$$

2. Suponhamos agora que a execução do algoritmo termina com $\vartheta_{r}<1-1 /(\log t)$. Portanto $r<(\log t) / 2$. Temos então

$$
\begin{aligned}
1-t^{-1 / 3}<\vartheta & =1-1 / t-c \sqrt{t_{r} / n_{r}} \leq 1-1 / \log t \\
-t_{r}^{-1 / 3} & <-1 / \log t \\
t_{r}^{-1 / 3} & >1 / \log t \\
1 / t_{r} & >1 /(\log t)^{3}
\end{aligned}
$$

portanto

$$
\begin{gathered}
\frac{n_{r}}{\left(t_{r}+1\right)}>c \frac{n_{r}}{t_{r}} \\
>c \frac{n_{r}}{(\log t)^{3}}>c \frac{n}{(\log t)^{3}} \frac{1}{2^{r}} \geq c \frac{n}{\sqrt{t}(\log t)^{3}}>c n \frac{\log t}{t}
\end{gathered}
$$

para $t$ suficientemente grande.

Os resultados expostos acima resumem a estratégia básica do método probabilístico que seguiremos neste trabalho. Na próxima seção vamos apresentar a versão para o caso de hipergrafos, enfatizando com um pouco mais de detalhe esta estratégia. 


\subsection{Hipergrafos de cintura maior que 4}

Vamos detalhar um pouco mais a estratégia apresentada na seção anterior, desta vez na versão para hipergrafos. Na primeira subseção descrevemos a transformação usada neste caso e na subseção seguinte as considerações necessárias para a prova algorítmica. Precisamos introduzir alguma notação.

Definição 2.2.1 Seja $\mathcal{F}=(V, \mathcal{F})$ um hipergrafo sobre $n$ vértices. Dado $x \in V$ definimos $\mathcal{F}_{x}=\{F \subset V \backslash\{x\}: F \cup\{x\} \in \mathcal{F}\}$. Dizemos que $\mathcal{F}$ é regular se para $1 \leq i \leq k$ temos que $d_{i}(x)=d_{i}(y)$ para todo $x, y \in V$. Dizemos que a cintura $g(\mathcal{F})$ de $\mathcal{F}$ é pelo menos $h$ se $\mathcal{F}$ não tem circuitos de comprimento menor do que $h$.

No que resta da seção assumimos que $k$ é um inteiro fixo arbitrario, $\mathcal{F}$ é um $(k+1)$ grafo sobre $n$ vértices e que toda aresta $F$ de $\mathcal{F}$ satisfaz $2 \leq|F| \leq k+1$. Enunciamos agora o resultado central desta seção.

Teorema 2.2.2 (Ajtai, Komlós, Pintz, Spencer e Szemerédi, '82; [1]) Seja dado um $(k+1)$-grafo $\mathcal{F}$ sobre $n$ vértices tal que para algum $t_{0}=t_{0}(k)$ e algum $n_{0}=n_{0}(k, t)$ valem

(i) $g(\mathcal{F}) \geq 5$,

(ii) $\Delta(\mathcal{F}) \leq t^{k}$, onde $t \geq t_{0}(k)$,

(iii) $n \geq n_{0}(k, t)$.

Então

$$
\alpha(\mathcal{F}) \geq c_{k} \frac{n}{t}(\log t)^{1 / k}
$$

Veremos rapidamente que este teorema implica uma versão menos restritiva como segue.

Corolário 2.2.3 (Alon, Lefmann e Rödl, '91; [3]) Seja dado um $(k+1)$-grafo $\mathcal{H}$ sobre $n$ vértices tal que

(i) $g(\mathcal{H}) \geq 5$

(ii) $\Delta(\mathcal{H}) \leq t^{k}$, onde $t \geq t_{0}(k)$.

Então

$$
\alpha(\mathcal{H}) \geq c_{k} \frac{n}{t}(\log t)^{1 / k} .
$$


Demonstração. Seja $\mathcal{H}$ um $(k+1)$-grafo sobre $n$ vértices satisfazendo as hipóteses do corolário e seja $m$ um inteiro positivo tal que

$$
m>\frac{n_{0}(k, t)}{n} .
$$

Consideremos o hipergrafo $\mathcal{F}_{m}$ formado por $m$ cópias de $\mathcal{H}$, disjuntas nos vértices. Então $\mathcal{F}_{m}$ tem $m n>n_{0}(k, t) / n$ vértices e satisfaz as condições $(i),($ ii $)$ e $($ iii $)$ do Teorema 2.2 .2 e portanto

$$
m \alpha(\mathcal{H})=\alpha\left(\mathcal{F}_{m}\right)>c_{k} \frac{n m}{t}(\log t)^{1 / k}
$$

assim,

$$
\alpha(\mathcal{H})>c_{k} \frac{n}{t}(\log t)^{1 / k}
$$

Recentemente este resultado foi melhorado no sentido de não ser preciso desconsiderar circuitos de comprimento 4 . Eis a nova versão.

Teorema 2.2.4 (Duke, Lefmann e Rödl, '95; [5]) Sejam dados um inteiro $k \geq 2 e$ um $(k+1)$-grafo $\mathcal{F}$ sobre $n$ vértices tais que

(i) $g(\mathcal{F}) \geq 3$,

(ii) $\Delta(\mathcal{F}) \leq t^{k}$, onde $t \geq t_{0}(k)$,

(iii) $n \geq n_{0}(k, t)$.

Então

$$
\alpha(\mathcal{F}) \geq c_{k} \frac{n}{t}(\log t)^{1 / k}
$$

Demonstração. Denotamos por $\mu_{3}$ o número de 3 -circuitos de $\mathcal{F}$ e por $\mu_{4}$ o número de 4-circuitos de $\mathcal{F}$.

Para limitar superiormente $\mu_{3}$ consideremos um vértice qualquer $x$ e vejamos quantos triângulos podem contê-lo. Duas arestas $F_{1}$ e $F_{2}$ incidentes a $x$ podem ser escolhidas de, no máximo, $\left(\begin{array}{c}t^{k} \\ 2\end{array}\right)$ formas. Para definir a terceira aresta do 3-circuito precisamos escolher um vértice $y \in F_{1}$ e outro $z \in F_{2}$. Portanto

$$
\mu_{3} \leq n\left(\begin{array}{c}
t^{k} \\
2
\end{array}\right) k^{2} \leq c_{3} n t^{2 k} .
$$

Já que $\mathcal{F}$ não tem 2-circuitos, escrevemos $F_{x, y}=F_{1}$ e $F_{x, z}=F_{2}$ para ressaltar que cada par de vértices determina univocamente a aresta que os contém.

Vamos agora limitar superiormente $\mu_{4}$. Consideremos um vértice qualquer $x$ e vejamos quantos quadrados podem contê-lo. Pela observação anterior, um tal quadrado fica 
definido ao escolher três outros vértices $y, z$ e $w$. Estes vértices determinam as arestas $F_{x y}, F_{x z}, F_{y w}$ e $F_{z w}$ do quadrado. Como antes, as arestas $F_{x y}$ e $F_{x z}$ podem ser escolhidas de, no máximo, $\left(\begin{array}{c}t^{k} \\ 2\end{array}\right)$ formas. Nestas arestas, precisamos escolher $y \in F_{1}$ e $z \in F_{2}$. Temos ainda, no máximo, $t^{k}$ escolhas para $F_{y, w}$ e $k$ escolhas para o $w$ em $F_{y, w}$ que vai determinar $F_{x, w}$. Portanto

$$
\mu_{4} \leq n\left(\begin{array}{c}
t^{k} \\
2
\end{array}\right)(k+1) t^{k}(k+1)^{2} \leq c_{4} n t^{3 k}
$$

Seja $Y \subset V$ tal que

$$
\mathrm{P}(x \in Y)=p=t^{\epsilon-1}, \quad \text { para todo } x \in V
$$

onde $0<\epsilon<(k-1) /(4 k-1)$. Então $|Y| \sim B(n, p)$ e portanto

$$
|Y| \sim \text { pn, quase sempre. }
$$

Sejam agora $\mu_{3}(Y)$ e $\mu_{4}(Y)$ as v.a. que contam respectivamente o número de 3-circuitos e de 4 -circuitos de $\mathcal{F}[Y]$. Então, para $i=3,4$, segue que

$$
\begin{aligned}
\mathrm{E}\left(\mu_{i}(Y)\right) & =\mu_{i} p^{i k} \\
& \leq c_{i} n t^{(i-1) k} p^{i k} \\
& =c_{i} t^{-(k-1)+\epsilon i k-\epsilon} p n \\
& =o(p n)
\end{aligned}
$$

pela escolha de $\epsilon$.

Denotando o número esperado de arestas em $Y$ por $\mathrm{E}(\mathcal{F}[Y])$, temos

$$
\mathrm{E}(\mathcal{F}[Y])=p^{k}|\mathcal{F}| \leq \frac{1}{k} p^{k} t^{k-1} n
$$

e pela desigualdade de Markov, $\mathrm{P}(X \leq \lambda \mu) \geq 1-1 / \lambda$, com $\lambda=2$, obtemos

$$
\mathrm{P}\left(|\mathcal{F}[Y]| \leq \frac{2}{k} p^{k} t^{k-1} n\right) \geq 0.5
$$

Já que dois eventos, cada um com probabilidade maior o igual que meio, devem ter interseção não vazia, concluimos que existe um $Y \subset V$ que satisfaz ambas afirmativas (7) e (8).

Agora, apagamos um vértice de cada 3-circuito e de cada 4-circuito em $Y$, e obtemos um subconjunto $Y_{0} \subset Y$, com $Y_{0} \sim p n$, tal que o subhipergrafo induzido $\mathcal{F}\left[Y_{0}\right]$ de $\mathcal{F}$ tem cintura $g\left(\mathcal{F}\left[Y_{0}\right]\right) \geq 5$.

Observemos que o grau médio de $\mathcal{F}\left[Y_{0}\right]$ satisfaz

$$
\frac{(k+1)\left|\mathcal{F}\left[Y_{0}\right]\right|}{n p} \leq \frac{2}{(k+1)}(k+1) \frac{p^{k+1} t^{k+1} n}{p n}=2 p^{k} t^{k},
$$


assim, os vértices de grau maior que $5 p^{k} t^{k}$ tem grau "grande" no seguinte sentido. Para cada vértice $x \in Y_{0}$, a desigualdade de Markov garante

$$
\mathrm{P}\left(d_{x} \geq 5 p^{k} t^{k}=(5 / 2) 2 p^{k} t^{k}\right) \leq 2 / 5
$$

e portanto a quantidade de vértices de $Y_{0}$ que tem grau maior ou igual a $5 p^{k} t^{k}$ é menor ou igual a $(2 / 5)\left|Y_{0}\right| \leq 0.5 n p$ e depois de apagá-los, obtemos um conjunto $Y_{1} \subset Y_{0}$, com $\left|Y_{1}\right| \geq p n / 2$, que induz um subhipergrafo $\mathcal{F}\left[Y_{1}\right]$ de $\mathcal{F}\left[Y_{0}\right]$ que satisfaz

$$
\Delta\left(\mathcal{F}\left[Y_{1}\right]\right)=t^{k} \leq 5 p^{k} t^{k}=5 t^{\epsilon k} .
$$

Então, pelo Corolário 2.2.3 obtemos

$$
\alpha(\mathcal{F}) \geq \alpha\left(\mathcal{F}\left[Y_{1}\right]\right) \geq c_{k} \frac{Y_{1}}{t_{1}}\left(\log t_{1}\right)^{1 / k} \geq c_{k} \frac{n}{t}(\log t)^{1 / k} .
$$




\subsubsection{A transformação}

Descrevemos brevemente a transformação que utilizaremos na prova do resultado central desta seção.

Lema 2.2.5 (A transformação; [1]) Seja dado um hipergrafo $\mathcal{F}=(V, \mathcal{F})$ e consideremos um conjunto $C \subset V$. Definimos

$$
\begin{aligned}
D & =\left\{x \in V: F \subset C \text { para algum } F \in \mathcal{F}_{x}\right\}, \\
I & =C \cap \bar{D}, \\
V^{*} & =\bar{C} \cap \bar{D}, \\
\mathcal{F}^{*} & =\left\{F^{\prime}=F \cap V^{*}: F \in \mathcal{F}, F \subset C \cup V^{*}, F \cap V^{*} \neq \emptyset\right\} .
\end{aligned}
$$

Então o hipergrafo $\mathcal{F}^{*}=\left(V^{*}, \mathcal{F}^{*}\right)$ satisfaz

(i) $\alpha(\mathcal{F}) \geq \alpha(\mathcal{F}[C])+\alpha\left(\mathcal{F}^{*}\right) \geq|I|+\alpha\left(\mathcal{F}^{*}\right)$,

(ii) $\left(V^{*}, \mathcal{F}\left[V^{*}\right]\right) \subset\left(V^{*}, \mathcal{F}^{*}\right)$,

(iii) $\left(V^{*}, \mathcal{F}^{*}\right)$ não contém hiperarestas unitárias e todas as $(k+1)$-arestas de $\mathcal{F}^{*}$ são $(k+1)$-arestas de $\mathcal{F}$.

Demonstração. (i) Com efeito, claramente $I$ é um conjunto independente de $\mathcal{F}$; agora suponhamos, para um absurdo, que existe um conjunto independente $J$ de $\mathcal{F}^{*}$ e uma aresta $F \in \mathcal{F}$ tal que $F \subset I \cup J$. Mas então $F \cap V^{*} \in \mathcal{F}^{*}$, o qual contradiz o fato de ser $J$ independente em $\mathcal{F}^{*}$.

(ii) Decorre simplesmente do fato de $I$ ser um conjunto independente.

(iii) Pela definição de $\mathcal{F}^{*}$, toda aresta contida em $V^{*}$ é uma aresta de $\mathcal{F}^{*}$.

(iv) Se $\{x\}=F^{\prime}=F \cap V^{*}$ é uma aresta unitária de $\mathcal{F}^{*}$ então $x \in V^{*}$, porém também deveria existir $F \in \mathcal{F}_{x}$ contida totalmente em $C$ ou seja $x \in D$, o qual é uma contradição. A outra afirmativa é imediata.

$\mathrm{Na}$ construção do algoritmo, os elementos do conjunto $C$ da transformação serão escolhidos ao acaso.

Definição 2.2.6 Dizemos que um conjunto de vértices $C \subset V$ tem distribuição $V(p)$, com $0<p<1$ se

$$
\mathrm{P}(x \in C)=p
$$

e esses eventos são mutuamente independentes. 
Lema 2.2.7 Seja dado um $(k+1)$-grafo regular $\mathcal{F}=(V, \mathcal{F})$ com $n$ vértices, cintura $g(\mathcal{F}) \geq 5$ e grau máximo $\leq t^{k}$. Considere $C \subset V$ com distribuição $V(p)$ com $p=1 / t e$ sejam I e $\left(V^{*}, \mathcal{F}^{*}\right)$ como em (2.2.5). Então

$$
\begin{aligned}
|I| & \sim \frac{n}{\mathrm{et}} \\
\left|V^{*}\right| & \sim \frac{n}{\mathrm{e}},
\end{aligned}
$$

quase sempre.

Da definição do conjunto $I$ em (2.2.5) segue que os eventos $\{x \in I\}$ e $\{y \in I\}$ são independentes se a distância entre $x$ e $y$ é maior do que 2 no hipergrafo $\mathcal{F}$. Ademais, a condição da cintura ser maior do que 4 implica que os vértices à distância menor ou igual a 2 são "poucos"; daí temos que $|I|$ tem distribuição "quase" binomial com $n$ experimentos e probabilidade de sucesso $p \sim 1$ /et. A tese para $|I|$ do Lema 1.2 .7 segue da desigualdade de Chebyshev. O argumento para $\left|V^{*}\right|$ é análogo.

A primeira afirmativa do Lema 1.2.7 e (i) do Lema 2.2.5 implicam o seguinte.

Corolário 2.2.8 Seja dado um $(k+1)$-grafo regular $\mathcal{F}=(V, \mathcal{F})$ com n vértices, cintura $g(\mathcal{F}) \geq 5$ e grau máximo $\leq t^{k}$. Então

$$
\alpha(\mathcal{F})>\frac{n}{\mathrm{e} t} .
$$

Lema 2.2.9 Seja dado um $(k+1)$-grafo regular $\mathcal{F}=(V, \mathcal{F})$ com $n$ vértices, cintura $g(\mathcal{F}) \geq 5$ e grau máximo $\leq t^{k}$. Considere $C \subset V$ com distribuição $V(p)$ com $p=1 / t e$ sejam I e $\left(V^{*}, \mathcal{F}^{*}\right)$ como em (2.2.5). Então

$$
d_{i}^{*}(x) \sim\left(\begin{array}{c}
k \\
i
\end{array}\right)\left(\frac{t}{\mathrm{e}}\right)^{i}, \quad 1 \leq i \leq k,
$$

quase sempre.

Do esboço da prova do Lema 1.2.7 sabemos que $\left|V^{*}\right|$ segue basicamente uma distribuição binomial. Algo similar acontece com o $i$-grau $d_{i}^{*}(x)$ de $x$ em $\mathcal{F}^{*}$. Cada $(k+1)$-aresta incidente a $x$ em $\mathcal{F}$ pode se transformar numa $(i+1)$-aresta das $\left(\begin{array}{c}k \\ i\end{array}\right)$ possíveis em $\mathcal{F}^{*}$, e a probabilidade da transformação em cada caso é aproximadamente $p^{k-i} \mathrm{e}^{-i}$. Como no máximo são $t^{k}$ experimentos, pois o grau máximo de $\mathcal{F}$ é $\leq t^{k}$, temos que $d_{i}^{*}(x)$ tem distribuição dominada por

$$
B\left(t^{k},\left(\begin{array}{c}
k \\
i
\end{array}\right) p^{k-i} \mathrm{e}^{-i}\right), \quad 1 \leq i \leq k,
$$

e a tese do Lema 1.2.9 segue da desigualdade de Chebyshev. 
Na construção a seguir, vamos requerer que os hipergrafos transformados sejam regulares (ou melhor, "o mais regulares possíveis" ou "quase-regulares"); o seguinte lema vai ser utilizado para manter essa propriedade entre um hipergrafo e seu transformado em cada passo da prova algorítmica.

Lema 2.2.10 (Quase-regularidade; [1]) Seja dado um hipergrafo $\mathcal{F}=(V, \mathcal{F})$ de $|V|=$ $n$ vértices com cintura $g(\mathcal{F}) \geq 5$ tal que para todo $x \in V$

$$
d_{i}(x) \leq a_{i}, \quad 1 \leq i \leq k .
$$

Então existe $\mathcal{F}^{+} \supset \mathcal{F}$ tal que

(i) $g\left(\mathcal{F}^{+}\right) \geq 5$

(ii) $d_{i}^{+}(x) \leq a_{i}$ para todo $1 \leq i \leq k$ e todo $x$, onde $d_{i}^{+}(x)$ denota o $i$-grau de $x$ em $\mathcal{F}^{+}$,

(iii) o conjunto $B=\left\{x: d_{i}^{+}(x) \neq a_{i}\right.$, algum $\left.i\right\}$ tem cardinalidade $|B| \leq k^{2} b^{3}$, onde $b=1+\sum_{i=1}^{k} i a_{i}$.

Demonstração. Seja $\mathcal{F}^{+}$o hipergrafo maximal tal que $\mathcal{F}^{+} \supset \mathcal{F}$ e satisfaz $(i)$ e $(i i)$.

Definimos

$$
B_{i}=\left\{x: d_{i}^{+}(x) \leq a_{i}\right\}
$$

de modo que

$$
B=\cup_{i=1}^{k} B_{i}
$$

Vamos ver que

$$
\left|B_{i}\right| \leq i b^{3}
$$

Com efeito, suponhamos que, para algum $i$, se satisfaz $\left|B_{i}\right| \leq i b^{3}$.

Já que $\left|\Gamma^{3}(x)\right| \leq b^{3}$ podemos definir uma nova aresta $\left\{x_{1}, \ldots, x_{i+1}\right\}$, escolhendo inductivamente um elemento arbitrário $x_{j}$ de $\Gamma^{3}\left(x_{s}\right)$ para $s<j$.

Então $\mathcal{F}^{+} \cup\left\{x_{1}, \ldots, x_{i+1}\right\}$ é hipergrafo sem circuitos de comprimento 3 e 4 e satisfazendo a condição (ii) o qual contradiz a escolha de $\mathcal{F}+$.

Portanto

$$
|B| \leq \sum_{i=1}^{k}\left|B_{i}\right|=b^{3} k(k+1) / 2
$$

e temos (iii).

Lema 2.2.11 Seja dado um hipergrafo regular $\mathcal{F}=(V, \mathcal{F})$ com $n$ vértices, cintura $g(\mathcal{F}) \geq$ 5 , e com

$$
d_{i}(x)=\alpha_{i}\left(\begin{array}{c}
k \\
i
\end{array}\right) t^{i}, \quad 1 \leq i \leq k
$$


Considere $C \subset V$ com distribuição $V(p)$ com $p=w / t$, onde $w$ satisfaz $w=O(\log t)$, $e$ sejam I e $\left(V^{*}, \mathcal{F}^{*}\right)$ como em (2.2.5). Então existem números reais $\alpha_{i}^{*}(1 \leq i \leq k)$ e $\beta>0$ tais que $\left(V^{*}, \mathcal{F}^{*}\right)$ tem $n^{*}=n \beta$ vértices e

$$
d_{i}^{*}(x) \sim \alpha_{i}^{*}\left(\begin{array}{c}
k \\
i
\end{array}\right)\left(t^{*}\right)^{i}, \quad 1 \leq i \leq k,
$$

quase sempre.

O tal $\beta$ vai ser escolhido tal que $\beta \sim \mathrm{e}^{-1}$. Considerações análogas ao Lema 1.2.7 permitem obter que, também neste caso, quase sempre,

$$
\begin{gathered}
|I| \sim n p \beta=\frac{n}{t} \beta w, \\
\left|V^{*}\right| \sim n \beta,
\end{gathered}
$$

e como esboçado para o Corolário 1.2.8 pode-se provar que $\alpha(\mathcal{F})>(n / t) \beta w$.

Além disso, dado $x \in V$, o número $d_{i j}^{*}(x)$ de $(j+1)$-arestas incidentes a $x$ em $\mathcal{F}$ que são transformadas em $(i+1)$-arestas incidentes a $x$ em $\mathcal{F}^{*}$ segue uma distribuição binomial de $d_{j}(x)$ experimentos e probabilidade de sucesso $\left(\begin{array}{l}j \\ i\end{array}\right) p^{j-i} \beta^{i}$, i.e. $d_{i j}^{*}(x)$ tem distribuição

$$
B\left(d_{j}(x),\left(\begin{array}{c}
j \\
i
\end{array}\right) p^{j-i} \beta^{i}\right) \text {. }
$$

Daí a tese do Lema 1.2.10 segue pela desigualdade de Chebyshev com $t^{*}=t \beta \mathrm{e}$

$$
\alpha^{*}=\sum_{j=1}^{k} \alpha_{j}\left(\begin{array}{c}
k-i \\
j-i
\end{array}\right) w^{j-i} .
$$




\subsubsection{O algoritmo}

Nesta subseção vamos apresentar resultados que garantem o funcionamento do algoritmo da prova do Teorema 1.2.2. Esta subseção é uma revisão da discussão do parágrafo 2 de $[1]$.

Apresentamos a seguir a construção algorítmica do conjunto independente que garante a tese do Teorema 1.2.2.

Algoritmo 2.2.12 Entrada: Um $(k+1)$-grafo regular $\mathcal{F}=(V, \mathcal{F})$ com $n$ vértices, cintura $g(\mathcal{F}) \geq 5$ e $d_{k}(x) \leq t^{k}$

Saída: Um conjunto independente I de tamanho $\geq c_{k}(n / t)(\log t)^{1 / k}$.

1. Ponha $\left(V_{0}, \mathcal{F}_{0}\right)=(V, \mathcal{F})$.

2. Para $s=0$ até $s=\lceil 0.01 \log t\rceil$ faça

$w=w_{s}=(s+1)^{1 / k}-s^{1 / k}$.

Seja $C \subset V_{s}$ com distribuição $V(p)$, onde $p=p_{s}=w_{s} / t_{s}$.

$C$ define $I=I_{s}$ e $\left(V_{s+1}, \mathcal{F}_{s+1}\right)=\left(V_{s}^{*}, \mathcal{F}_{s}^{*}\right)$ como em (2.2.5).

Substitua $\mathcal{F}_{s+1}$ pela sua extensão $\mathcal{F}_{s+1}^{+}$segundo (2.2.10).

3. Devolva $I=\bigcup_{s=0}^{\lceil 0.01 \log t\rceil} I_{s}$.

A seguir argumentamos que o algoritmo acima calcula efetivamente o que precisamos.

Lema 2.2.13 (Os invariantes) Em cada iteração s do Algoritmo 2.2.12 temos, quase sempre,

(i) $\beta_{s} \sim \mathrm{e}^{-1}$,

(ii) $\left|V_{s}\right|=n_{s} \sim n \mathrm{e}^{-s}, t_{s} \sim t \mathrm{e}^{-s}, v_{s} \sim s^{1 / 2}$,

(iii) $d_{i}=\alpha_{i}\left(\begin{array}{c}k \\ i\end{array}\right) t_{s}^{i}$, onde $\alpha_{i}=v_{s}^{k-i}$ para todo $x \in V_{s}$,

(iv) $\left|I_{s}\right| \sim(n / \mathrm{e} t) w_{s}$.

Demonstração. A prova deste lema é indutiva.

Caso base: Ao início da execução do algoritmo, já que o hipergrafo de entrada é $(k+1)$-uniforme e regular, os Lemas 1.2 .7 e 1.2 .9 e o Corolário 1.2.8 garantem que a execução da transformação de (2.2.5) mantém os invariantes.

Passo indutivo: Nas seguintes iterações, o algoritmo usa (2.2.10) para preservar a regularidade, mas o hipergrafo transformado não é mais $(k+1)$-uniforme; neste caso, o Lema 1.2.10 garante que a transformação (2.2.5) conserva os invariantes do Lema 1.1.12, completando a indução. 
Corolário 2.2.14 (Certificado) O conjunto I devolvido pelo Algoritmo 2.2.12 é um conjunto independente de $(V, \mathcal{F})$ e satisfaz

$$
|I| \geq c_{k}(n / t)(\log t)^{1 / k} .
$$

Demonstração. A tese é conseqüência de (iv) do Lema 1.2.12 e do fato de que $|I|$ só depende da soma telescópica

$$
\sum_{s=0}^{\lceil 0.01 \log t\rceil} w_{s}=\sum_{s=0}^{\lceil 0.01 \log t\rceil}(s+1)^{1 / k}-s^{1 / k}=\lceil 0.01 \log t\rceil=c_{k}^{\prime} \log t .
$$

Com efeito,

$$
|I|=\sum_{s=0}^{\lceil 0.01 \log t\rceil}\left|I_{s}\right| \sim \sum_{s=0}^{\lceil 0.01 \log t\rceil}(n / e t) w_{s}=c_{k}^{\prime}(n / e t) \log t .
$$




\subsection{Conseqüências}

\subsubsection{Uma seqüência de Sidon densa}

Definição 2.3.1 Uma seqüência de Sidon é uma seqüência de números inteiros positivos $S=\left\{a_{1}<a_{2}<\ldots<a_{k}<\ldots\right\}$ (finita ou infinita) tal que todo inteiro $m$ tem, no máximo, uma representação da forma

$$
m=a_{i}+a_{j}, \quad \operatorname{com} a_{i}, a_{j} \in S, i<j .
$$

Denotamos com $s(n)$ o número máximo de elementos de $\{1, \ldots, n\}$ que formam uma seqüência de Sidon. Se $S$ é uma seqüência infinita de Sidon, denotamos $\operatorname{com} f_{S}(n)$ o número de elementos de $S$ não maiores que $n$.

Vimos no Capítulo 1 que o crescimento de $s(n)$ é aproximadamente o crescimento da raiz quadrada de $n$, conforme $n$ cresce indefinidamente. O resultado para grafos sem triângulos da Seção 2.1 foi desenvolvido como ferramenta para a investigação de $f_{S}(n)$. Agora vamos apresentar essa aplicação.Remetemos o leitor ao Capítulo 1 para o histórico dos avanços na pesquisa deste problema.

Precisamos de um lema técnico antes de continuar. Dados dois inteiros $x, y \operatorname{com} x \leq y$ dizemos que a tripla $(x, y, x+y)$ é um triângulo geral.

Dizemos que um conjunto $A$ gera um triângulo geral $(x, y, x+y)$ se $x \in A-A=$ $\left\{a-a^{\prime}: a, a^{\prime} \in A\right\}, y \in A-A$ e $x+y \in A-A$.

Lema 2.3.2 Dados $N$ triângulos gerais e um intervalo $(a, a+n)$ existe um conjunto diferença $D \subset(a, a+n)$ tal que

(i) $|D| \geq \sqrt{n} / 3$,

(ii) no máximo $10 \mathrm{~N} / \sqrt{n}$ dos triângulos gerais dados podem encontrar-se em $D$ como diferenças consecutivas $(d, d+x, d+x+y)$ para algum $d$.

Demonstração. Como a cada triângulo geral $(x, y, x+y)$ corresponde o triângulo geral $(x-a, y-a, x+y-2 a)$ podemos assumir que $a=0$.

A estratégia para obter as afirmativas é construir $m=\sqrt{n} / 10$ conjuntos diferença $D_{1}, \ldots, D_{m}$ de tamanho $\sqrt{n} / 3$, todos eles contidos em $(0, n)$ e tais que nenhum par deles compartilha um triângulo geral. Algum desses conjuntos $D_{j}$ deve satisfazer $(i i)$.

Seja um número primo $p=4 k+1$ tal que

$$
0.8 \sqrt{n}<p<0.9 \sqrt{n}
$$

Definimos

$$
D_{j}=\left\{p x+\left(j x^{2}\right): 1 \leq x<p \text { tais que }\left(j x^{2}\right)<p / 2\right\}, \quad j=1, \ldots, m
$$


onde $(t)$ é o mínimo resíduo positivo de $t \bmod p$.

$D_{j}$ é um conjunto diferença. Com efeito, sejam

$$
u_{i}=p x_{i}+\left(j x_{i}^{2}\right) \in D_{j}, \quad i=1,2,3,4 .
$$

Assumindo que $u_{1} \neq u_{2}$ e $u_{3} \neq u_{4}$ supomos, por absurdo, que

$$
u_{1}-u_{2}=u_{3}-u_{4}
$$

daí temos

$$
\begin{aligned}
& x_{1}-x_{2}=x_{3}-x_{4}, \\
& x_{1}^{2}-x_{2}^{2}=x_{3}^{2}-x_{4}^{2} .
\end{aligned}
$$

Dessas equações obtemos

$$
x_{1}+x_{2}=x_{3}+x_{4},
$$

e somando a esta equação a primeira equação temos

$$
x_{1}=x_{3} \quad e \quad x_{2}=x_{4} .
$$

E, portanto, $u_{1}=u_{3}$ e $u_{2}=u_{4}$.

Cada triângulo geral está contido num só $D_{j}$. Seja um triângulo geral $T=$ $(a p+c, b p+d,(a+b) p+(c+d))$, com $0 \leq c<p, 0 \leq d<p$ e suponhamos que

$$
T \subset D_{j}, \quad \text { para algum } j \in[m]
$$

Vamos mostrar que tal $j$ é único. Com efeito, se

$$
p x_{i}+\left(j x_{i}^{2}\right), \quad j=1,2,3,
$$

são os elementos de $D$ correspondentes a $T$, digamos $\left(p x_{1}+\left(j x_{1}^{2}\right), p x_{2}+\left(j x_{2}^{2}\right), p x_{3}+\left(j x_{3}^{2}\right)\right)=$ $(d, d+a p+c, d+(a+b) p+c+d)$, para algum $d$. Nessas condições

$$
\begin{gathered}
p x_{1}+\left(j x_{1}^{2}\right)=d, \\
p x_{2}+\left(j x_{2}^{2}\right)=d+a p+c, \\
p x_{3}+\left(j x_{3}^{2}\right)=(a+b) p+c+d, \\
\\
p x_{1}+\left(j x_{1}^{2}\right)-p x_{2}-\left(j x_{2}^{2}\right)=a p+c, \\
p x_{3}+\left(j x_{3}^{2}\right)-p x_{2}-\left(j x_{2}^{2}\right)=b p+d
\end{gathered}
$$

e, portanto,

$$
x_{1}-x_{2}=a \bmod p, \quad\left(j x_{2}^{2}\right)-\left(j x_{1}^{2}\right)=c \bmod p,
$$




$$
\begin{gathered}
x_{2}-x_{3}=b \bmod p, \quad\left(j x_{2}^{2}\right)-\left(j x_{3}^{2}\right)=d \bmod p . \\
\left\{x_{1}-x_{2}\right\}^{-1}=a^{-1} \bmod p, \\
\left\{x_{2}-x_{3}\right\}^{-1}=b^{-1} \bmod p, \\
\left(j\left\{x_{1}-x_{2}\right\}\left\{x_{1}+x_{2}\right\}\right)=c \bmod p, \\
\left(j\left\{x_{2}-x_{3}\right\}\left\{x_{2}+x_{3}\right\}\right)=d \bmod p,
\end{gathered}
$$

portanto,

$$
\begin{gathered}
\left(j\left\{x_{1}+x_{2}\right\}\right)=a^{-1} c \bmod p, \\
\left(j\left\{x_{2}+x_{3}\right\}\right)=b^{-1} d \bmod p . \\
\left(j\left\{x_{1}-x_{3}\right\}\right)=\left\{a^{-1} c-b^{-1} d\right\} \bmod p \\
x_{1}-x_{3}=\{a-b\} \bmod p, \\
j=\{a-b\}^{-1}\left\{a^{-1} c-b^{-1} d\right\} \bmod p .
\end{gathered}
$$

Se passaram vinte anos até se conseguir construir uma seqüência de Sidon de crescimento mais rápido: em 1981, Ajtai, Komlós e Szemerédi [2] apresentaram uma construção probabilística de uma seqüência de Sidon mais densa que as anteriores; seu resultado é baseado no Teorema 2.1.7. Eis o enunciado. 
Teorema 2.3.3 (Ajtai, Komlós e Szemerédi, '81; [2]) Existe uma seqüência de Sidon $S$ tal que

$$
f_{S}(n)>\frac{1}{10^{500}}(n \log n)^{1 / 3}
$$

para todo $n \in \mathrm{N}$.

Demonstração. Por indução matemática.

Caso Base. Deve-se garantir a existência de um conjunto $B_{i_{0}}$, para $i_{o}$ suficientemente grande, tal que

(i) $B_{i_{0}} \subset\left[2.10^{i_{0}}, 3.10^{i_{0}}\right]$,

(ii) $\left|B_{i_{0}}\right|=\left\lfloor\left(i_{0}^{1 / 3} 10^{i_{0} / 3}\right) / 10^{500}\right\rfloor$,

(iii) $B_{i_{0}}$ é um conjunto diferença,

(iv) O conjunto $A_{i_{0}}=B_{i_{0}}$ gera menos do que $10^{1,26 i_{0}}$ triângulos gerais.

Hipótese indutiva. Seja $i>i_{0}$ e suponhamos que existam $B_{i_{0}}, B_{i_{0+1}}, \ldots, B_{i-1}$ tais que para todo $h$ tal que $i_{0} \leq h<i$ se satisfaz

(i) $B_{h} \subset\left[2.10^{h}, 3.10^{h}\right]$,

(ii) $\left|B_{h}\right|=\left\lfloor\left(h^{1 / 3} 10^{h / 3}\right) / 10^{500}\right\rfloor$,

(iii) $B_{h}$ é um conjunto diferença,

(iv) O conjunto $A_{h}=\cup_{j \leq h} B_{j}$ gera menos do que $10^{1.26 h}$ triângulos gerais,

(v) Para nenhum par $b, b^{\prime} \in B_{h}$ acontece $b-b^{\prime} \in A_{h-1}-A_{h-1}$.

A idéia é mostrar que a seqüência de elementos dos $B_{i}$ formam uma sequiência de Sidon com a densidade desejada.

Passo Indutivo. Pelo Lema 2.3.2 sabemos que existe um conjunto diferença $D \subset$ $\left[2.10^{i}, 3.10^{i}\right]$, tal que

(i) $D=10^{i / 2} / 3$,

(ii) No máximo $10 \cdot 10^{1,26(i-1)} \cdot 10^{-0,5 i}=10^{0,76 i+0,5}<10^{1,26 i}$ dos triângulos gerados por $A_{i-1}$, podem ser encontrados em $D$ como diferenças consecutivas $(d, d+x, d+x+y)$.

Escolhemos $B \subset D$ pondo

$$
\mathrm{P}(x \in B)=p=5 \cdot 10^{-0,15 i}, \quad \forall x \in D,
$$


sendo esses eventos independentes. Temos, portanto, que $|B|$ segue uma distribuição binomial $B\left((1 / 3) 10^{i / 2}, 5.10^{-0,15 i}\right)$ e assim

$$
|B| \sim(5 / 3) 10^{0,35 i}, \quad \text { quase sempre. }
$$

Pondo $\epsilon=2 / 5$ na desigualdade de Chebyshev, $\mathrm{P}((1-\epsilon) \mu<X<(1+\epsilon) \mu)>1-\left(1 / n \epsilon^{2} p\right)$, obtemos

$$
\mathrm{P}\left(10^{0,35 i}<|B|<(7 / 3) 10^{0,35 i}\right)>0,9 .
$$

Seja agora $G T R_{i}$ o número de triângulos gerais gerados por $A_{i-1} \cup B$ e $G T R_{i-1}$ o número de triângulos gerais gerados por $A_{i-1}$. Então, para $i>600$,

$$
\mathrm{E}\left(G T R_{i}-G T R_{i-1}\right)<\left(4.10^{0,35 i}\right)^{3}+\left(4.10^{0,35 i}\right)^{4} p<(1 / 20) 10^{1,26 i},
$$

já que pelo menos dois dos 6 inteiros que determinam cada triângulo geral devem estar em $B_{i}$. Portanto, pela desigualdade de Markov, $\mathrm{P}(X \leq \lambda \mu) \geq 1-(1 / \lambda)$, tomando $\lambda=10$ obtemos

$$
\mathrm{P}\left(G T R_{i}-G T R_{i-1}<(1 / 2) 10^{1,26 i}\right) \geq 0,9
$$

e, portanto,

$$
\mathrm{P}\left(G T R_{i}<10^{1,26(i-1)}+(1 / 2) 10^{1,26 i}<10^{1,26 i}\right) \geq 0,9 .
$$

Seja agora $G_{i}=\left(B, E G_{i}\right)$ onde, dados $b, b^{\prime} \in B$ temos que $b b^{\prime} \in E G_{i}$ se e somente se $b-b^{\prime} \in A_{i-1}-A_{i-1}$. Definimos $T R$ como o número de triângulos em $G_{i}$. Então, para $i>100$, obtemos

$$
\mathrm{E}(T R)<10^{0,77 i} p^{3}=125.10^{0,32 i} .
$$

Então pela desigualdade de Markov, tomando $\lambda=10$ e $i>300$, temos

$$
\mathrm{P}\left(T R<10^{0,33 i}\right)>0,9 .
$$

Como os eventos (1), (2), (3), (4) tem probabilidade $\geq 0.9$ então eles têm interseção não vazia e, portanto, existe um $B$ satisfazendo $(i),(i i i)$, (iv) (por (3)).

Podemos então retirar os triângulos de $G_{i}$ sem diminuir demais a quantidade de vértices. Por abuso de notação, chamamos tambem $G_{i}$ esse grafo sem triângulos. Temos que o número de arestas de $E G_{i}$ é limitado por $\left|A_{i-1}-A_{i-1}\right| p^{2}=O\left(\left|A_{i-1}\right|^{2} p^{2}\right)$ e como

$$
\begin{gathered}
\left|A_{i-1}\right|^{2} \leq\left(\sum_{k=i_{0}}^{i-1} \frac{k^{1 / 3} 10^{k / 3}}{100}\right)^{2} \leq\left(\frac{c\left((i-1) 10^{i-1}\right)^{1 / 3}}{100}\right)^{2} \leq \frac{c^{2}}{100}\left(i 10^{i}\right)^{1 / 3} \\
\mathrm{E}\left(E G_{i}\right)<(1 / 5000) i^{2 / 3} 10^{2 i / 3} p^{2}<(1 / 100) i^{2 / 3} 10^{(2 / 3-0,3) i}
\end{gathered}
$$


e pela desigualdade de Markov, $\operatorname{com} \lambda=10$, temos

$$
\mathrm{P}\left(E G_{i}<(1 / 10) i^{2 / 3} 10^{(2 / 3-0.3) i}\right) \geq 0,9 .
$$

Seja $t$ o grau médio de $G_{i}$. Então, por (5) e por (1)

$$
t=\frac{2\left|E G_{i}\right|}{\left|V G_{i}\right|}<\frac{2}{10} \frac{i^{2 / 3} 10^{(2 / 3-0.3) i}}{10^{0.35 i}}<\frac{2}{10} i^{2 / 3} 10^{(2 / 3-0.65) i}
$$

e pelo Teorema $2.1 .7, G_{i}$ contém pelo menos

$$
\begin{gathered}
2.10^{0.35 i} \frac{\log t}{t}>10^{(0.35+0.65) i} \frac{10 \log t}{i^{2 / 3} 10^{2 i / 3}} \\
=10^{i / 3} \frac{10 \log t}{i^{2 / 3}} \sim \frac{i}{i^{2 / 3}} 10^{i / 3}
\end{gathered}
$$

vértices independentes. Tomando extremos temos

$$
2.10^{0.35 i} \frac{\log t}{t}>i^{1 / 3} 10^{i / 3}
$$

vértices independentes; desses vértices $\left(1 / 10^{500}\right) i^{1 / 3} 10^{i / 3}$ vão formar $B_{i}$ satisfazendo $(i i) \mathrm{e}$ $(v)$. 


\subsubsection{Um limitante inferior para o problema de Heilbronn}

Recordemos do Capítulo 1 a conjectura de Heilbronn. Consideremos o disco $D$ de área 1 no plano e um conjunto $S=\left\{p_{1}, \ldots, p_{n}\right\}$ de $n$ pontos de $D$. Seja $\Delta(S)$ a área mínima de um triângulo cujos vértices são 3 pontos diferentes de $D$. Heilbronn $(\leq 51)$ colocou o problema de calcular

$$
\Delta(n)=\max \Delta(S)
$$

onde $S$ toma valores sobre todos os conjuntos de $n$ pontos em $D$. A primeira estimativa para $\Delta(n)$ é

$$
\Delta(n)=O\left(n^{-1}\right),
$$

e Heilbronn conjecturou que o valor correto era $\Delta(n)=O\left(n^{-2}\right)$.

Conjectura 2.3.4 (Heilbronn, $\leq$ '51) Para alguma constante absoluta $c>0$, toda configuração de $n$ pontos no disco unitário contém necessariamente um triângulo de área menor que $\mathrm{cn}^{-2}$.

Erdős, em [18], mostrou que $\Delta(n) \neq o\left(n^{-2}\right)$ provando assim que se essa conjectura fosse válida então o resultado seria o "melhor possível".

Porém a conjectura de Heilbronn é falsa e foi refutada em 1982 por Komlós, Pintz e Szemerédi [14], que provaram que $\Delta(n)$ não só não é limitada por $c n^{-2}$ (para alguma constante $c$ ), como seu crescimento é, pelo menos, mais veloz que esse limite vezes un fator logarítmico.

Teorema 2.3.5 (Komlós, Pintz e Szemerédi, '82; [14]) Para todo inteiro $n$ existe uma configuração de $n$ pontos no disco unitário que não contém triângulos de área menor que

$$
c_{1}(\log n) / n^{2}
$$

onde $c_{1}>0$ é uma constante absoluta.

Demonstração. Definamos os números $t, n_{1}, \Delta$ pondo

$$
\begin{aligned}
t & =n_{1}^{1 / 100}, \\
n & =c_{2}\left(n_{1} / t\right)(\log t)^{1 / 2}, \\
\Delta & =c_{3}(\log n) / n^{2} .
\end{aligned}
$$

Escolhamos ao acaso $n_{1}$ pontos no disco unitário, independentemente e uniformemente e definamos o 3-grafo $\mathcal{F}$ pondo 3 destes pontos $a, b, c$ numa aresta $\{a, b, c\}$ de $\mathcal{F}$ se e somente se os pontos $a, b, c$ formam um triângulo de área menor do que $\Delta$.

Vamos limitar a probabilidade de que três destes pontos, $a, b, c$ formem um triângulo de área menor do que $\Delta$. Para tanto, expressamos essa probabilidade em termos do Teorema de Probabilidade Total

$$
\mathrm{P}(\operatorname{Area}(a, b, c) \leq \Delta)=\int_{-\infty}^{+\infty} \mathrm{P}(\operatorname{Area}(a, b, c) \leq \Delta|| b-a \mid=r) f_{|b-a|}(r) d r
$$


onde,

$$
\mathrm{P}(\operatorname{Area}(a, b, c) \leq \Delta|| b-a \mid=r)=\int_{\operatorname{Area}(a, b, c) \leq \Delta} f_{\operatorname{Area}(a, b, c)|| b-a \mid}(a \mid r) d a
$$

para limitar superiormente a probabilidade condicional consideramos que a base do triângulo é formada pelos pontos $a, b$ e que estes pontos se encontram a distância $r$; a altura $h$ do terceiro ponto $c$ deve satisfazer $h r / 2<\Delta$. Nestas condições, a área onde o ponto $c$ pode tomar valores é limitada pela área de um retângulo de base máxima $\leq 2 / \sqrt{\pi}$ e altura $2 h<4 \Delta / r$, portanto,

$$
\mathrm{P}(\operatorname{Area}(a, b, c) \leq \Delta|| b-a \mid=r) \leq 2 h r<(4 \Delta / r)(2 / \sqrt{\pi}) .
$$

Por outro lado, a distribuição do comprimento da base do triângulo pode-se limitar como segue

$$
\mathrm{P}(r \leq|b-a| \leq r+\Delta r) \leq \pi(r+\Delta r)^{2}-\pi r^{2}
$$

portanto,

$$
\frac{F(r+\Delta r)-F(r)}{\Delta r} \leq \pi(2 r+\Delta r)
$$

e tomando limite quando $\Delta r \rightarrow 0$ obtemos

$$
F^{\prime}(r)=f(r)=2 \pi r .
$$

Portanto,

$$
\mathrm{P}(\text { Area }(a, b, c) \leq \Delta) \leq \int_{0}^{2 / \sqrt{\pi}} \frac{8 \Delta}{r \sqrt{\pi}} 2 \pi r d r=32 \Delta<t^{2} / n_{1}^{2} .
$$

Sejam agora

$$
X_{a, b, c}= \begin{cases}1 & \text { se } a, b, c \in V \text { e Area }(a, b, c) \leq \Delta, \\ 0 & \text { caso contrário }\end{cases}
$$

e $X$ igual ao número de triângulos de área menor ou igual que $\Delta$. Então

$$
X=\sum_{a, b, c \in V} \operatorname{e\operatorname {Area}(a,b,c)\leq \Delta } X_{a, b, c},
$$

portanto,

$$
\begin{gathered}
\mathrm{E}(X)=\sum_{a, b, c \in V \text { e } \operatorname{Area}(a, b, c) \leq \Delta} \mathrm{E}\left(X_{a, b, c}\right) \\
=\left(\begin{array}{l}
n \\
3
\end{array}\right) \mathrm{P}(a, b, c \in V \text { e } \operatorname{Area}(a, b, c) \leq \Delta)<\frac{n^{3}}{6} \frac{t^{2}}{n_{1}^{2}},
\end{gathered}
$$

portanto, o valor esperado do número de triângulos de área menor que $\Delta$ é menor que $n_{1} t^{2} / 6$ e, assim, o valor esperado do grau médio $t^{(3)}$ satisfaz

$$
t^{(3)}=\frac{1}{n} \sum_{x \in V} d^{(3)}(x)=\frac{3|\mathcal{F}|}{n},
$$


portanto,

$$
\mathrm{E}\left(t^{(3)}\right)=\frac{3 \mathrm{E}(|\mathcal{F}|)}{n}<\frac{3}{n} \frac{n t^{2}}{6}=\frac{t^{2}}{2} .
$$

Agora vamos mostrar que podem-se descartar os circuitos de comprimento 2 do 3-grafo $\mathcal{F}$, sem diminuir muito o número de vértices.

Com efeito, já que

$$
\mathrm{P}(x, y \in V \text { tais que }|y-x|<d)=\int_{D} \mathrm{P}(y \in V:|y-x|<d \mid x \in V) f_{X}(x) d x
$$

onde

$$
\mathrm{P}(y \in V:|y-x|<d \mid x \in V)=\int_{y:|y-x|<d} f_{|y-x| \mid x}(s \mid x) d s .
$$

Já que esta última integral é limitada superiormente por $\pi d^{2}$ e como a área $D$ é 1 , temos

$$
\mathrm{P}(x, y \in V \text { tais que }|y-x|<d) \leq d^{2} \pi
$$

então o número de pares de vértices a distância menor que $d=n^{-0.6}$ em $\mathcal{F}$ é, com alta probabilidade, menor que

$$
\left(\begin{array}{c}
n_{1} \\
2
\end{array}\right) d^{2} \pi<2 n_{1}^{0.8}
$$

descartemos estes vértices de $\mathcal{F}$.

Que duas arestas formem um 2-circuito significa que foram escolhidos quatro vértices $a, b, c, d$ tais que as arestas $\{a, b, c\}$ e $\{a, b, d\}$ formam triângulos de área menor do que $\Delta$. Sendo que a eleição dos vértices é independente então as probabilidades de eleger $c$ e $d$ se multiplicam e, pela desigualdade de Markov, temos que o número de 2-circuitos de $\mathcal{F}$ é, com alta probabilidade, menor que

$$
c_{4} n_{1}^{4} \int_{d}^{2} \frac{\Delta^{2}}{r^{2}} 2 \pi r d r<c_{5} t^{4} \log n_{1}<n_{1}^{0.1}
$$

Apagando os vértices em cada um destes circuitos, obtemos um 3-grafo (que denotamos também por $\mathcal{F}$ ), com cintura maior ou igual do que 3. Portanto, pelo Teorema 2.2.4 este 3-grafo contém pelo menos

$$
c_{2}\left(n_{1} / t\right)(\log t)^{1 / 2}=n
$$

vértices independentes, ou seja, existem $n$ pontos no disco unitário $D$ que não tem triângulos de área menor do que

$$
c_{1}(\log n) / n^{2}
$$




\section{Capítulo 3}

\section{Uma Técnica de Rödl}

O ano 1985 foi prolífico em resultados sobre empacotamentos e coberturas de hipergrafos; Os resultados que comentaremos foram desenvolvidos a partir da prova original de Rödl de uma conjectura de Erdős e Hanani. Na primeira seção deste capítulo vamos nos concentrar em expressar, na linguagem algorítmica das Seções 2.1 e 2.2 do Capitulo 2, uma versão muito elegante de Pippenger do resultado de Frankl e Rödl; o resultado de Rödl será obtido como corolário desse teorema de Pippenger na segunda seção; a terceira seção deste capítulo é incluida como um histórico geral dos sucessivos avanços desta técnica desenvolvidos a partir do resultado original de Rödl,

Definição 3.0.6 Dado um hipergrafo $\mathcal{F}=(V, \mathcal{F})$ e $X \subset V$, definimos

$$
\mathcal{F}(X)=\{F \in \mathcal{F}: X \subset F\}
$$

$e$

$$
d(X)=|\mathcal{F}(X)|
$$

No caso de ser $X=\{x\}$, escrevemos simplesmente $\mathcal{F}(x)$ e $d(x)$ e se $X=\{x, y\}$ escreve$\operatorname{mos} \mathcal{F}(x, y)$ e d $(x, y)$.

Uma cobertura (de vértices por arestas ) $\mathcal{K}$ em $\mathcal{F}$ é um conjunto de hiperarestas de $\mathcal{F}$ tal que cada vértice está, no mínimo, numa hiperaresta de $\mathcal{K}$. Um empacotamento $\mathcal{P}$ em $\mathcal{F}$ é um conjunto de hiperarestas tal que todo vértice está, no máximo, numa hiperaresta de $\mathcal{P}$. Uma cobertura ou um empacotamento é perfeito se cada vértice está em exatamente uma de suas hiperarestas. Neste caso os conceitos coincidem. Denotamos por $\nu(\mathcal{F})$ a cardinalidade de um empacotamento máximo e por $\operatorname{Cob}(\mathcal{F})$ a cardinalidade de uma cobertura minima de $\mathcal{F}$.

Definição 3.0.7 Dado um hipergrafo $\mathcal{F}=(V, \mathcal{F})$ o índice cromático $\chi^{\prime}(\mathcal{F})$ é o número minimo de empacotamentos nos quais as hiperarestas de $\mathcal{F}$ podem ser particionadas. Denotamos com $\phi(\mathcal{F})$ o tamanho máximo de uma coleção disjunta de coberturas de $\mathcal{F}$. 
Os resultado de Pippenger que apresentaremos foi derivado do seguinte teorema de Frankl e Rödl.

Teorema 3.0.8 (Frankl e Rödl, '85; [10]) Sejam dados $\epsilon>0$ arbitrário, um r-grafo $\mathcal{F}=(V, \mathcal{F})$ sobre $n$ vértices e a>3 um número real. Existe um número real positivo $\delta=\delta(\epsilon)$ tal que se para algum $D$ temos

(i) $d(x) \sim_{\delta} D$ para todo $x \in V$,

(ii) $d(x, y)<D /(\log n)^{a}$ para todo $x, y, x \neq y$,

então, para todo $n>n_{0}(\delta)$, temos

$$
\operatorname{Cob}(\mathcal{F}) \leq \frac{n}{r}(1+\epsilon)
$$

\subsection{A versão restrita de Pippenger do teorema de Frankl e Rödl}

\subsubsection{A transformação}

Alguns anos depois, em 1989, Pippenger e Spencer [16] obtêm um resultado bem mais forte que o de Frankl e Rödl, sob hipóteses menos restritivas. Parafraseando o sumário do artigo: Numa coleção de $r$-grafos com grau mínimo assintótico ao grau máximo $\Delta$ e tal que o número de hiperarestas contendo qualquer par de vértices fixos é desprezível em relação ao grau máximo, tem-se que o índice cromático é assintótico ao grau máximo. Isto quer dizer que as hiperarestas podem ser particionadas em empacotamentos ou coberturas quase-perfeitos.

A seguir apresentamos o novo resultado.

Lema 3.1.1 (A transformação) Sejam fixos $r, \epsilon>0$ e K. Então para todo $\delta^{\prime}>0$, existe um $\delta>0$ para o qual vale o seguinte. Se $n>n_{0}\left(r, \delta^{\prime}, \epsilon, K\right)$ e $D>D_{0}\left(r, \delta^{\prime}, \epsilon, K\right)$ e é dado um r-grafo $\mathcal{F}=(V, \mathcal{F})$ sobre $n$ vértices satisfazendo

(i) $d(x)<K D$ para todo $x \in V$,

(ii) $d(x) \sim_{\delta} D$ vale para, no mínimo, $(1-\delta) n$ vértices,

(iii) $d(x, y)<\delta D$ para todo $x \neq y$,

então existe $\mathcal{R} \subset \mathcal{F}$ tal que pondo $V^{*}=V \backslash \bigcup \mathcal{R}$, e $\mathcal{F}^{*}=\left.\mathcal{F}\right|_{V^{*}}$, temos

(iv) $|\mathcal{R}| \sim_{\delta^{\prime}} \epsilon n / r$,

(v) $\left|\bigcup \mathcal{F}^{*}\right| \sim_{\delta^{\prime}} n \mathrm{e}^{-\epsilon}$, 
(vi) $d^{*}(x) \sim_{\delta^{\prime}} D \mathrm{e}^{-\epsilon(r-1)}$ vale para, no mínimo, $\left(1-\delta^{\prime}\right)\left|\cup \mathcal{F}^{*}\right|$ dos vértices de $\mathcal{F}^{*}$.

Demonstração. Define-se o subhipergrafo $\mathcal{R}$ pondo

$$
\mathrm{P}(F \in \mathcal{R})=p=\epsilon / D
$$

sendo estes eventos independentes. Daí as provas de $(i v),(v)$ e $(v i)$ do Lema 2.3.4 seguem o seguinte roteiro: aproxima-se a esperança da variável de interesse até um erro pequeno $\delta$; depois estima-se a variância da variável para aplicar a desigualdade de Chebyshev e mostrar que a variável está próxima do valor de sua esperança com alta probabilidade. Sem entrar em todos os detalhes, vamos esboçar as aproximações das esperanças.

Considere

$$
X=\{x \in V: d(x)=(1 \pm \delta) D\}
$$

de $(i i)$ sabemos $|X| \geq(1-\delta) n$ e portanto

$$
\frac{(1-\delta)^{2} D}{r} \leq \frac{\sum_{x \in X} d(x)}{r} \leq \frac{(1+\delta) D|X|}{r}
$$

para $x \in X$. Por outro lado, de $(i)$, temos

$$
0 \leq \frac{\sum_{x \notin X} d(x)}{r} \leq \frac{K D|\bar{X}|}{r}
$$

portanto

$$
(1-\delta)^{2} \frac{D n}{r} \leq \frac{\sum_{x \in V} d(x)}{r}=|\mathcal{F}| \leq \frac{D}{r}\{(1+\delta)|X|+|\bar{X}|\} K
$$

considerando $|X| \leq n$ e $|\bar{X}| \leq \delta n$ temos

$$
(1-(2-\delta) \delta) \frac{D n}{r} \leq|\mathcal{F}| \leq \frac{D n}{r}\{1+(1+K) \delta\}
$$

tomando $\delta_{1}=\min \{(2-\delta) \delta,(1+K) \delta\}$ obtemos

$$
\left(1-\delta_{1}\right) \frac{D n}{r} \leq|\mathcal{F}| \leq \frac{D n}{r}\left(1+\delta_{1}\right)
$$

i.e.

$$
|\mathcal{F}| \sim_{\delta_{1}} \frac{D n}{r} .
$$

(iv) Da afirmativa anterior temos,

$$
|| \mathcal{F}\left|-\frac{D n}{r}\right| p \leq \delta_{1} \frac{D n}{r} p
$$


e já que $|\mathcal{R}| \sim B(|\mathcal{F}|, p)$ segue que

$$
\left|\mathrm{E}(|\mathcal{R}|)-\frac{\epsilon n}{r}\right| \leq \delta_{1} \frac{\epsilon n}{r}
$$

i.e.

$\mathrm{ou}$

$$
\begin{gathered}
\left(1-\delta_{1}\right) \frac{\epsilon n}{r} \leq \mathrm{E}(|\mathcal{R}|) \leq\left(1+\delta_{1}\right) \frac{\epsilon n}{r} \\
\mathrm{E}(|\mathcal{R}|) \sim_{\delta_{1}} \frac{\epsilon n}{r}
\end{gathered}
$$

$$
\mathrm{E}(|\mathcal{R}|)=\left(1 \pm \delta_{1}\right) \frac{\epsilon n}{r}
$$

Para aproximar $|\mathcal{R}|$ por sua variância temos que escolher um $\lambda$ tal que

$$
|| \mathcal{R}|-\mathrm{E}(|\mathcal{R}|)| \leq \lambda \mathrm{E}(|\mathcal{R}|)
$$

i.e.

$$
(1-\lambda) \mathrm{E}(|\mathcal{R}|) \leq|\mathcal{R}| \leq(1+\lambda) \mathrm{E}(|\mathcal{R}|)
$$

da aproximação para $\mathrm{E}(|\mathcal{R}|)$

$$
\left(1-\delta_{1}\right)(1-\lambda) \frac{\epsilon n}{r} \leq(1-\lambda) \mathrm{E}(|\mathcal{R}|) \leq|\mathcal{R}| \leq(1+\lambda) \mathrm{E}(|\mathcal{R}|) \leq(1+\lambda)\left(1+\delta_{1}\right) \frac{\epsilon n}{r}
$$

portanto basta tomar $\delta_{2}=\delta_{1}+\lambda+\delta_{1} \lambda$ e $\lambda \leq \delta_{1}$, temos

$$
\left(1-\delta_{2}\right) \frac{\epsilon n}{r} \leq|\mathcal{R}| \leq\left(1+\delta_{2}\right) \frac{\epsilon n}{r}
$$

e assim

$$
|\mathcal{R}| \sim_{\delta_{2}} \frac{\epsilon n}{r}
$$

$\mathrm{Ou}$

$$
|\mathcal{R}|=\left(1 \pm \delta_{2}\right) \frac{\epsilon n}{r}
$$

Já que para a variância temos

$$
\operatorname{Var}(|\mathcal{R}|)=|\mathcal{F}| p(1-p) \leq \frac{2 n \epsilon}{r}
$$

então, pela desigualdade de Chebyshev, obtemos

$$
\begin{gathered}
\mathrm{P}\left(|\mathcal{R}|=\left(1 \pm \delta_{2}\right) \frac{\epsilon n}{r}\right)=\mathrm{P}(|| \mathcal{R}|-\mathrm{E}(|\mathcal{R}|)| \leq \lambda \mathrm{E}(|\mathcal{R}|)) \\
\geq 1-\frac{\operatorname{Var}(|\mathcal{R}|)}{(\lambda \mathrm{E}(|\mathcal{R}|))^{2}}=1-\frac{2 r}{\lambda^{2} \epsilon n\left(1-\delta_{1}\right)} \rightarrow 1
\end{gathered}
$$

para algum $\delta_{2}$, quando $n \rightarrow+\infty$. Daí $(i v)$ vale quase sempre. 
(v) Definimos, para cada $x \in V^{*}$, as variáveis

$$
Y_{x}= \begin{cases}1 & \text { se } x \notin \bigcup \mathcal{R} \\ 0 & \text { se } x \in \bigcup \mathcal{R} .\end{cases}
$$

Portanto

$$
\left|\bigcup \mathcal{F}^{*}\right|=\sum_{x \in V^{*}} Y_{x}
$$

Já que temos

$$
\mathrm{E}\left(Y_{x}\right)=(1-p)^{d(x)}
$$

$\mathrm{e}$

$$
\mathrm{e}^{-2 p d(x)} \leq(1-p)^{d(x)} \leq \mathrm{e}^{-p d(x)}
$$

então para os vértices $x$ tais que $d(x)=D(1 \pm \delta)$

$$
\begin{aligned}
\mathrm{e}^{-2 p(1-\delta) D} \leq \mathrm{e}^{-2 p d(x)} & \leq(1-p)^{d(x)} \leq \mathrm{e}^{-p d(x)} \leq \mathrm{e}^{-p(1+\delta) D} \\
\mathrm{e}^{-2 \epsilon(1-\delta)} & \leq(1-p)^{d(x)} \leq \mathrm{e}^{-\epsilon(1+\delta)}
\end{aligned}
$$

Tomando $\delta_{3}=\max \left\{\mathrm{e}^{-\epsilon(1+\delta)}-\mathrm{e}^{-\epsilon}, \mathrm{e}^{-\epsilon}-\mathrm{e}^{-\epsilon(1-\delta)}\right\}$ (e para isto $\delta$ tem que ser pequeno o bastante) obtemos

$$
\begin{gathered}
\mathrm{e}^{-\epsilon}-\delta_{3} \leq \mathrm{e}^{-2 \epsilon(1-\delta)} \leq(1-p)^{d(x)} \leq \mathrm{e}^{-\epsilon(1+\delta)} e s \leq \mathrm{e}^{-\epsilon}+\delta_{3} \\
\mathrm{e}^{-\epsilon}-\delta_{3} \leq(1-p)^{d(x)} \leq \mathrm{e}^{-\epsilon}+\delta_{3} \\
\left|\mathrm{E}\left(Y_{x}\right)-\mathrm{e}^{-\epsilon}\right| \leq \delta_{3},
\end{gathered}
$$

ou melhor, tomando $\delta^{*}=\delta_{3} / e^{-\epsilon}$ temos

$$
\mathrm{E}\left(Y_{x}\right) \sim_{\delta_{3}^{*}} \mathrm{e}^{-\epsilon} .
$$

Por outro lado, já que os outros vértices satisfaçem $0 \leq \mathrm{E}\left(Y_{x}\right) \leq 1$ obtemos

$$
\begin{aligned}
n(1-\delta)\left(\mathrm{e}^{-\epsilon}-\delta_{3}\right) & \leq \sum_{x: d(x)=D(1 \pm \delta)} \mathrm{E}\left(Y_{x}\right) \leq \mathrm{E}\left(\left|\cup \mathcal{F}^{*}\right|\right)=\sum_{x: d(x)=D(1 \pm \delta)} \mathrm{E}\left(Y_{x}\right)+\sum_{x: d(x) \neq D(1 \pm \delta)} \mathrm{E}\left(Y_{x}\right) \\
& \leq \sum_{x: d(x)=D(1 \pm \delta)}\left(\mathrm{e}^{-\epsilon}+\delta_{3}\right)+\sum_{x: d(x) \neq D(1 \pm \delta)} 1 \leq n\left(\mathrm{e}^{-\epsilon}+\delta_{3}\right)+\delta n
\end{aligned}
$$

portanto

$$
n \mathrm{e}^{-\epsilon}(1-\delta)\left(1-\delta_{3} \mathrm{e}^{\epsilon}\right) \leq \mathrm{E}\left(\left|\bigcup \mathcal{F}^{*}\right|\right) \leq n \mathrm{e}^{-\epsilon}\left(1+\left(\delta_{3}+\delta\right) \mathrm{e}^{\epsilon}\right),
$$

ou seja,

$$
\mathrm{E}\left(\left|\bigcup \mathcal{F}^{*}\right|\right)=\Theta(n) .
$$

Note que, tomando $\delta_{4}=\max \left\{\delta+(1-\delta) \delta_{3} \mathrm{e}^{\epsilon},\left(\delta_{3}+\delta\right) \mathrm{e}^{\epsilon}\right\}$ temos

$$
n \mathrm{e}^{-\epsilon}\left(1-\delta_{4}\right) \leq \mathrm{E}\left(\left|\cup \mathcal{F}^{*}\right|\right) \leq n \mathrm{e}^{-\epsilon}\left(1+\delta_{4}\right)
$$


isto é

$$
\mathrm{E}\left(\left|\bigcup \mathcal{F}^{*}\right|\right)=n \mathrm{e}^{-\epsilon}\left(1 \pm \delta_{4}\right)
$$

$\mathrm{Ou}$

$$
\mathrm{E}\left(\left|\bigcup \mathcal{F}^{*}\right|\right) \sim_{\delta_{4}} n \mathrm{e}^{-\epsilon}
$$

Limitase superiormente a variância de $\left|\cup \mathcal{F}^{*}\right|$, utilizando-se de

$$
\operatorname{Var}\left(\left|\bigcup \mathcal{F}^{*}\right|\right)=\sum_{x \in V} \operatorname{Var}\left(Y_{x}\right)+\sum_{x, y \in V: x \neq y} \sum \operatorname{Cov}\left(Y_{x}, Y_{y}\right) .
$$

Note que

$$
\begin{gathered}
\operatorname{Cov}\left(Y_{x}, Y_{y}\right)=\mathrm{E}\left(Y_{x} Y_{y}\right)-\mathrm{E}\left(Y_{x}\right) \mathrm{E}\left(Y_{y}\right) \\
=(1-p)^{d(x)+d(y)-d(x, y)}-(1-p)^{d(x)+d(y)}=(1-p)^{d(x)+d(y)}\left((1-p)^{-d(x, y)}-1\right) \\
\leq(1-p)^{-d(x, y)}-1<\mathrm{e}^{p d(x, y)}-1<\mathrm{e}^{\epsilon \delta}-1<\delta_{5}
\end{gathered}
$$

e esse $\delta_{5}$ pode ser tão pequeno quanto desejado ajustando-se $\delta$.

Para o primeiro somando temos

$$
\sum_{x \in V} \operatorname{Var}\left(Y_{x}\right) \leq \sum_{x \in V} \mathrm{E}\left(Y_{x}\right)=\mathrm{E}\left(\left|\bigcup \mathcal{F}^{*}\right|\right)=o\left(\left(\mathrm{E}\left(\left|\bigcup \mathcal{F}^{*}\right|\right)\right)^{2}\right)
$$

Portanto, dado $\delta_{5}$ existe um $n_{0}$ tal que se $n \geq n_{o}$ então

$$
\mathrm{E}\left(\left|\bigcup \mathcal{F}^{*}\right|\right) \leq \delta_{5}\left(\mathrm{E}\left(\left|\bigcup \mathcal{F}^{*}\right|\right)\right)^{2}
$$

e assim podemos limitar a variância como segue

$$
\operatorname{Var}\left(\left|\bigcup \mathcal{F}^{*}\right|\right) \leq \delta_{5}\left(\mathrm{E}\left(\left|\bigcup \mathcal{F}^{*}\right|\right)\right)^{2}+\delta_{5} n^{2}
$$

e já que $\mathrm{E}\left(\left|\cup \mathcal{F}^{*}\right|\right.$ é da ordem de $n^{2}$

$$
\leq \delta_{5}\left(\mathrm{E}\left(\left|\bigcup \mathcal{F}^{*}\right|\right)\right)^{2}+\delta_{5} C_{2}\left(\mathrm{E}\left(\left|\bigcup \mathcal{F}^{*}\right|\right)\right)^{2}
$$

para alguma constante $C_{2}$. Tomando $\delta_{6} \leq \delta_{5}\left(1+C_{2}\right)$, ainda temos que esse $\delta_{6}$ pode se tornar tão pequeno quanto desejado ajustando-se $\delta_{5}$ (que por sua vez se controla com $\delta \ldots$ ). Tomamos $\delta_{6} \ll \delta_{4}^{2}$ e obtemos

$$
\operatorname{Var}\left(\left|\bigcup \mathcal{F}^{*}\right|\right) \leq \delta_{6}\left(\mathrm{E}\left(\left|\bigcup \mathcal{F}^{*}\right|\right)\right)^{2}
$$

Vamos ajustar agora a proximidade de $\left|\cup \mathcal{F}^{*}\right|$ e a sua esperança para poder aplicar a desigualdade de Chebyshev. Para tal efeito, precisamos determinar um $\lambda$ tal que

$$
|| \bigcup \mathcal{F}^{*}\left|-\mathrm{E}\left(\left|\bigcup \mathcal{F}^{*}\right|\right)\right| \leq \lambda \mathrm{E}\left(\left|\bigcup \mathcal{F}^{*}\right|\right)
$$


Pela definição de $\delta_{4}$ temos

$n \mathrm{e}^{-\epsilon}\left(1-\delta_{4}\right)(1-\lambda) \leq \mathrm{E}\left(\left|\bigcup \mathcal{F}^{*}\right|\right)(1-\lambda) \leq\left|\bigcup \mathcal{F}^{*}\right| \leq \mathrm{E}\left(\left|\bigcup \mathcal{F}^{*}\right|\right)(1+\lambda) \leq n \mathrm{e}^{-\epsilon}\left(1+\delta_{4}\right)(1+\lambda)$

portanto basta tomar $\delta_{7}=\delta_{4}+\lambda+\delta_{4} \lambda$ e $\lambda \leq \delta_{4}$. Se $\lambda=\delta_{4}$ temos

$$
\begin{gathered}
n \mathrm{e}^{-\epsilon}\left(1-\delta_{7}\right) \leq\left|\bigcup \mathcal{F}^{*}\right| \leq n \mathrm{e}^{-\epsilon}\left(1+\delta_{7}\right) \\
\left|\bigcup \mathcal{F}^{*}\right| \sim_{\delta_{7}} n \mathrm{e}^{-\epsilon}
\end{gathered}
$$

ou

$$
\left|\bigcup \mathcal{F}^{*}\right|=\left(1 \pm \delta_{7}\right) n \mathrm{e}^{-\epsilon}
$$

Agora, aplicando a desigualdade de Chebyshev,

$$
\begin{gathered}
\mathrm{P}\left(\left|\bigcup \mathcal{F}^{*}\right|=n \mathrm{e}^{-\epsilon}\left(1 \pm \delta_{7}\right)\right)=\mathrm{P}\left(|| \bigcup \mathcal{F}^{*}\left|-\mathrm{E}\left(\left|\bigcup \mathcal{F}^{*}\right|\right)\right| \leq \lambda \mathrm{E}\left(\left|\bigcup \mathcal{F}^{*}\right|\right)\right) \\
\geq 1-\frac{\delta_{6}}{\lambda^{2}}=1-\frac{\delta_{6}}{\delta_{4}^{2}} \rightarrow 1
\end{gathered}
$$

pela escolha de $\delta_{6}$, para $n$ suficientemente grande. Daí $(v)$ vale quase sempre.

(vi) Começamos observando que dado $x \in V$ e $F_{0} \in \mathcal{F}$ com $x \in F_{0}$, temos

$$
\left|\left\{F \in \mathcal{F}: x \notin F, F \cap F_{0} \neq \emptyset\right\}\right| \sim_{\delta_{8}}(r-1) D .
$$

Pois, se $\{x, y\} \subset F$ então

$$
\left|\left\{F^{\prime} \in \mathcal{F}:\{x, y\} \subset F^{\prime},\right\}\right| \leq \delta D
$$

Portanto quase todas as arestas (a menos de $\delta D$ delas) incidentes aos outros vértices de $F$ (distintos de $x$ ), não contém $x$ e são em quantidade $(r-1) D-\delta D$.

Assumindo que $x \notin \cup \mathcal{R}$, observamos que $F_{0} \notin \mathcal{R}$ quando $F \notin \mathcal{R}$, para toda aresta $F$ que contém algum vertice $y \in F_{0}, y \neq x$. Consideremos agora o que acontece com as arestas do conjunto no $r$-grafo transformado $\mathcal{F}^{*}$. Nenhuma de tais arestas está em $\mathcal{R}$ com probabilidade

$$
\begin{gathered}
(1-p)^{(r-1) D} \sim_{\delta_{9}} \mathrm{e}^{-p(r-1) D}=\mathrm{e}^{-\epsilon(r-1)}, \\
\mathrm{P}\left(F \in \mathcal{F}^{*} \mid x \notin \mathcal{R}\right)=(1-p)^{(r-1) D}=\mathrm{e}^{-(r-1) \epsilon} \pm \delta_{10},
\end{gathered}
$$

$\operatorname{assim} d^{*}(x) \sim B\left((1 \pm \delta) D, \mathrm{e}^{-\epsilon(r-1)} \pm \delta_{10}\right) \mathrm{e}$

$$
\mathrm{E}\left(d^{*}(x)\right)=D\left(\mathrm{e}^{-\epsilon(r-1)} \pm \delta_{11}\right) .
$$

Para cada $F$ incidente a $x$ (tem mais ou menos $D$ deles), definimos

$$
X_{F}= \begin{cases}1 & \text { se } F \in \mathcal{F}^{*} \\ 0 & \text { caso contrario. }\end{cases}
$$


Daí

$$
\begin{aligned}
d^{*}(x) & =\sum_{F \in \mathcal{F}: x \in F} X_{F} \\
\mathrm{E}\left(d^{*}(x)\right) & =\sum_{F \in \mathcal{F}: x \in F} \mathrm{E}\left(X_{F}\right)
\end{aligned}
$$

e se $F, F^{\prime} \in \mathcal{F} \operatorname{com} x \in F$ e $x \in F^{\prime}$. Para $\operatorname{Sop}(F)$ sendo o número de arestas de $\mathcal{F}$ que incidem a $F$ e não estão em $\mathcal{R}$,

$$
\begin{gathered}
\operatorname{Cov}\left(X_{F}, X_{F^{\prime}}\right)=(1-p)^{\left|\operatorname{Sop}(F) \cup \operatorname{Sop}\left(F^{\prime}\right)\right|}-(1-p)^{2(r-1) D} \\
=(1-p)^{2(r-1) D}\left((1-p)^{- \text {número de arestas comuns }}-1\right) \\
=(1-p)^{2(r-1) D}\left(\mathrm{e}^{\epsilon(r-1)^{2} \delta}-1\right)<\delta_{12} .
\end{gathered}
$$

Portanto

$$
\begin{gathered}
\operatorname{Var}\left(d^{*}(x)\right)=\sum_{F: x \in F} \operatorname{Var}\left(X_{F}\right)+\sum_{F: x \in F} \sum_{F^{\prime}: x \in F^{\prime}} \operatorname{Cov}\left(X_{F}, X_{F^{\prime}}\right) \\
\leq \mathrm{E}\left(d^{*}(x)\right)+\sum_{F: x \in F} \sum_{F^{\prime}: x \in F^{\prime}} \delta_{12}=\mathrm{E}\left(d^{*}(x)\right)+\delta_{13} D^{2}
\end{gathered}
$$

e a desigualdade de Chebyshev garante $(v i)$.

\subsubsection{O algoritmo}

Teorema 3.1.2 (Pippenger, cf. Füredi, '88; [11]) Para todo inteiro $r \geq 2$ e números reais $K \geq 1$ e $\epsilon>0$ existe um $\delta>0$ para o qual vale o seguinte. Se, para algum $d$, o r-grafo $\mathcal{F}=(V, \mathcal{F})$ sobre $n$ vértices satisfaz

(i) $d(x)<K d$ para todo $x \in V$,

(ii) $d(x) \sim_{\delta} d$ vale para, no mínimo, $(1-\delta) n$ dos vértices,

(iii) $d(x, y)<\delta d$ para todo $x \neq y$,

então

$$
\operatorname{Cob}(\mathcal{F})<(n / r)(1+\epsilon)
$$

Para a demonstração consideremos o seguinte algoritmo.

Algoritmo 3.1.3 Entrada: $r \geq 2, K \geq 1, \epsilon>0$ e um $r$-grafo $\mathcal{F}$ satisfazendo (i), (ii) $e$ (iii) do Teorema 2.3.5.

Saída: Uma cobertura de $\mathcal{F}$.

1. Ponha $\left(V_{0}, \mathcal{F}_{0}\right)=(V, \mathcal{F})$, 


$$
\begin{aligned}
& \epsilon^{\prime}>0 \text { tal que } \epsilon^{\prime} /\left(1-\mathrm{e}^{-\epsilon^{\prime}}\right)+r \epsilon^{\prime}<1+\epsilon, \\
& t \in \mathrm{N} \text { o menor inteiro tal que }(1-\epsilon)^{t}<\epsilon, \\
& \delta_{0}>0 \text { tal que }\left(1+\delta_{0}\right)\left(\epsilon^{\prime} /\left(1-\mathrm{e}^{-\epsilon^{\prime}}\right)+r \epsilon^{\prime}\right)<1+\epsilon \\
& \text { e defina uma seqüência } \delta_{0}, \delta_{1}, \ldots, \delta_{t} \text { tal que } \delta_{s+1} \leq \delta_{s} \mathrm{e}^{-\epsilon^{\prime}(r-1)} .
\end{aligned}
$$

2. Para $s=0$ até $s=t-1$ faça

$$
\text { Calcule } \mathcal{R}_{s+1} \text { e } \mathcal{F}_{s+1}=\mathcal{F}_{s}^{*} \text { aplicando o Lema 2.3.4 a } \mathcal{F}_{s} \text {. }
$$

3. Para cada vértice não coberto por algum $\mathcal{R}_{s}$ escolha arbitrariamente uma aresta contendo-o e seja $\mathcal{R}_{t}$ o conjunto de tais arestas.

4. Devolva $\mathcal{R}=\bigcup_{s=0}^{t} \mathcal{R}_{s}$.

Lema 3.1.4 (Invariantes) Em cada iteração s do Algoritmo 3.1.3, temos

$$
\begin{aligned}
& \left|\mathcal{R}_{s}\right| \sim_{\delta_{s}} \epsilon \frac{n \mathrm{e}^{-\epsilon s}}{r}, \\
& \left|\bigcup \mathcal{F}_{s}\right| \sim_{\delta_{s}} n \mathrm{e}^{-\epsilon s} .
\end{aligned}
$$

As afirmativas deste lema decorrem por indução das afirmativas $(i v)$ e $(v)$, respectivamente, do Lema 2.3.4.

Lema 3.1.5 (Corretude) A cobertura $\mathcal{R}=\bigcup_{s=0}^{t} \mathcal{R}_{s}$, devolvida pelo Algoritmo $3.1 .3 \mathrm{sa}$ tisfaz

$$
|\mathcal{R}| \leq \frac{n}{r}(1+\epsilon) .
$$

Com efeito, pela primeira afirmativa do lema anterior e do fato de ser $\left|V_{s}\right| \sim_{\delta_{t}} n \mathrm{e}^{-\epsilon^{\prime} t}$, temos

$$
\begin{aligned}
|\mathcal{R}| & =\sum_{s=0}^{t-1} \mathcal{R}_{s}+\mathcal{R}_{t} \leq \sum_{s=0}^{t-1} \epsilon^{\prime} \frac{n}{r} \mathrm{e}^{-\epsilon^{\prime} s}+n \mathrm{e}^{-\epsilon^{\prime} t} \\
& =\frac{n}{r}\left\{\epsilon^{\prime} \frac{\left(1-\mathrm{e}^{-\epsilon^{\prime} t}\right)}{\left(1-\mathrm{e}^{-\epsilon^{\prime}}\right)}+r \mathrm{e}^{-\epsilon^{\prime} t}\right\} \leq \frac{n}{r}\left\{\frac{\epsilon^{\prime}}{\left(1-\mathrm{e}^{-\epsilon^{\prime}}\right)}+r \epsilon^{\prime}\right\},
\end{aligned}
$$

sendo a última desigualdade garantida pela escolha de $t$; de onde temos a tese, pela escolha de $\epsilon^{\prime}$. 


\subsection{Conseqüências: a conjectura de Erdős e Hanani}

Recordemos do Capítulo 1 a conjectura de Erdős e Hanani. Dados inteiros $n$, $k$ e $r$ $(r \leq k \leq n)$, definimos $M(k, r, n)$ como a cardinalidade de um sistema mínimo de $k$ conjuntos (subconjuntos de tamanho $k$ ) de $[n]=\{1, \ldots, n\}$ tal que todo $r$-conjunto de $[n]$ está contido em, no mínimo, um dos $k$-conjuntos do sistema. Analogamente denotamos por $m(k, r, n)$ a cardinalidade de um sistema maximo de $k$-conjuntos de $[n]$ tal que todo $r$-conjunto de $[n]$ está contido, no máximo, num $k$-conjunto do sistema.

Conjectura 3.2.1 (Erdős e Hanani, '63; [8]) Para todo $r \leq k$ fixos, temos

$$
\lim _{n \rightarrow+\infty} m(k, r, n)\left(\begin{array}{l}
k \\
r
\end{array}\right)\left(\begin{array}{l}
n \\
r
\end{array}\right)^{-1}=\lim _{n \rightarrow+\infty} M(k, r, n)\left(\begin{array}{l}
k \\
r
\end{array}\right)\left(\begin{array}{l}
n \\
r
\end{array}\right)^{-1}=1
$$

Foi para provar essa conjectura que Rödl [17], estabeleceu os resultados expostos na Seção 1 do Capítulo 2. Aqui comentamos como essa conjetura é conseqüência do Teorema de Frankl e Rödl na versão de Pippenger.

Consideremos o $\left(\begin{array}{l}k \\ r\end{array}\right)$-grafo $\mathcal{F}$ com vértices $[n]^{(r)}=\mathrm{K}_{r}^{n}$, onde um conjunto $F$ de $\left(\begin{array}{l}k \\ r\end{array}\right)$ arestas de $\mathrm{K}_{r}^{n}$ formam uma aresta de $\mathcal{F}$ se $\mathcal{F}[F] \cong \mathrm{K}_{r}^{k}$. Verifica-se então o seguinte.

$\operatorname{Dados}\left(\begin{array}{l}k \\ r\end{array}\right)>2, \epsilon>0$, existe $\delta>(k-r) /(n-r)$ e $K>0$ tal que

(i) $d_{\mathcal{F}}(x)<K\left(\begin{array}{l}n-r \\ k-r\end{array}\right)$ para todo $x \in[n]^{(r)}$,

(ii) $d_{\mathcal{F}}(x) \sim_{\delta}\left(\begin{array}{l}n-r \\ k-r\end{array}\right)$ para todo $x \in[n]^{(r)}$,

(iii) $d_{\mathcal{F}}(x, y)<\delta\left(\begin{array}{l}n-r \\ k-r\end{array}\right)$ para todos $x, y \in[n]^{(r)}, x \neq y$.

Então, pelo Teorema 2.3.5, temos

$$
\operatorname{Cob}(\mathcal{F})<\left(\begin{array}{l}
n \\
r
\end{array}\right)\left(\begin{array}{l}
k \\
r
\end{array}\right)^{-1}(1+\epsilon)
$$

Assim

$$
\operatorname{Cob}(\mathcal{F})=\left(\begin{array}{l}
n \\
r
\end{array}\right)\left(\begin{array}{l}
k \\
r
\end{array}\right)^{-1}(1+o(1))
$$

onde $o(1) \rightarrow 0$ quando $n \rightarrow+\infty$.

Seja $C$ uma tal cobertura (de vértices por arestas) de $\mathcal{F}$, digamos $C=\left\{F_{1}, \ldots, F_{\operatorname{Cob}(\mathcal{F})}\right\}$. Ponha $\mathcal{C}=\left\{V_{1}, \ldots, V_{\operatorname{Cob}(\mathcal{F})}\right\}$ onde $V_{i}$ é a união dos $r$-conjuntos que formam a aresta $F_{i}$ para todo $i$. Então $\mathcal{C}$ é um sistema de $k$-conjuntos de $[n]$ tal que todo $r$-conjunto de $[n]$ está contido em, no mínimo, um dos $k$-conjuntos de $\mathcal{C}$. Portanto

$$
\lim _{n \rightarrow+\infty} M(k, r, n)\left(\begin{array}{l}
k \\
r
\end{array}\right)\left(\begin{array}{l}
n \\
r
\end{array}\right)^{-1}=1 .
$$


Vamos construir agora um empacotamento que verifique o limitante inferior.

Com efeito, definimos

$$
\begin{gathered}
X=\left\{x \in[n]^{(r)}: \text { x é coberto pelo menos duas vezes por C }\right\} \\
P=\{F \in C: \exists x \in X, x \in F\} .
\end{gathered}
$$

A seguir consideramos os graus com respeito a $C$.

$$
\begin{gathered}
\sum_{x \in[n]^{(r)}} d_{C}(x)=|C|\left(\begin{array}{l}
k \\
r
\end{array}\right) \leq\left(\begin{array}{l}
n \\
r
\end{array}\right)(1+\epsilon) \\
\sum_{x \in[n]^{(r)}} d_{C}(x) \geq \sum_{x \in[n]^{(r)}} 1+\sum_{x \in X}\left(d_{C}(x)-1\right) \geq\left(\begin{array}{l}
n \\
r
\end{array}\right)+|X|
\end{gathered}
$$

portanto

$$
|X| \leq \epsilon\left(\begin{array}{l}
n \\
r
\end{array}\right)
$$

Consideremos agora

$$
\sum_{x \in[n]^{(r)}} d_{C}(x)=\sum_{x \notin X} 1+\sum_{x \in X} d_{C}(x) \geq(1-\epsilon)\left(\begin{array}{l}
n \\
r
\end{array}\right)+|P|
$$

do limitante superior obtemos

$$
|P| \leq 2 \epsilon\left(\begin{array}{l}
n \\
r
\end{array}\right) .
$$

Definimos agora o empacotamento $E=C \backslash P$ e temos

$$
\begin{gathered}
|E|=|C \backslash P|=|C|-|P| \\
\geq\left(\begin{array}{l}
n \\
r
\end{array}\right)\left(\begin{array}{l}
k \\
r
\end{array}\right)^{-1}-2 \epsilon\left(\begin{array}{l}
n \\
r
\end{array}\right) \geq\left(1-2 \epsilon\left(\begin{array}{l}
k \\
r
\end{array}\right)\right)\left(\begin{array}{l}
n \\
r
\end{array}\right)\left(\begin{array}{l}
k \\
r
\end{array}\right)^{-1}
\end{gathered}
$$

e portanto

$$
\lim _{n \rightarrow+\infty} m(k, r, n)\left(\begin{array}{l}
k \\
r
\end{array}\right)\left(\begin{array}{l}
n \\
r
\end{array}\right)^{-1}=1 .
$$




\subsection{Apêndice: Desenvolvimento histórico da técnica de Rödl}

\subsubsection{O trabalho seminal de Rödl e os comentários de Spencer}

Em 1985 Rödl [17] inaugura uma técnica na investigação de empacotamentos e coberturas de hipergrafos; apresentamos nesta subseção só os principais lemas auxiliares do artigo citado, deixando seu resultado central para ser derivado como conseqüência de resultados mais gerais que apresentaremos em seções posteriores.

Doravante denotaremos por $X^{(r)}$ a família dos subconjuntos de tamanho $r$ de um conjunto $X$.

Definição 3.3.1 Seja dado um r-grafo k-partido $\mathcal{F} \subset\left[\bigcup_{j=1}^{k} V_{j}\right]^{(r)}$ e $L \subset \bigcup_{j=1}^{k} V_{j}$ com $|L| \geq r$. Aqui e a seguir, assumimos que $V(\mathcal{F})=\bigcup_{j=1}^{k} V_{j}$ é sempre a k-partição associada a $\mathcal{F}$. Dizemos que $L$ é completo sobre $|L|$ vértices se $[L]^{(r)} \subset \mathcal{F}$, ou seja $\mathcal{F}[L] \cong \mathrm{K}_{r}^{|L|}$, o r-grafo completo sobre $|L|$ vértices. Se $L$ é um conjunto completo e $|L|=k$ dizemos que $\mathcal{F}[L] \cong \mathrm{K}_{r}^{k}$ é um $k$-ágono, e se $|L|<k$ denotamos com $\sigma^{L}(\mathcal{F})$ o número de $k$-ágonos de $\mathcal{F}$ contendo $L$.

Para todo $I \subset[k]$ com $|I|=r$, pomos $\rho_{I}(\mathcal{F})=\left|\mathcal{F}_{I}\right|$, onde

$$
\mathcal{F}_{I}=\left\{F \in \mathcal{F}: F \cap V_{i} \neq \emptyset \text {, para todo } i \in I\right\} .
$$

Neste caso vamos tomar ao acaso um sistema $\mathcal{K}$ de $k$-ágonos e definir como o $r$-grafo transformado $\mathcal{F}^{*}$ o formado pelas arestas não cobertas por nenhum $k$-ágono de $\mathcal{K}$.

Lema 3.3.2 (A transformação) Seja dado um r-grafo k-partido $\mathcal{F} \subset\left[\bigcup_{j=1}^{k} V_{j}\right]^{(r)}$ com $\left|V_{j}\right|=n$ para todo $j$, e suponha que $\rho$ e $\sigma_{l}(r \leq l \leq k-1)$ são reais positivos menores do que 1 tais que

(i) $\rho_{I}(\mathcal{F}) \sim \rho n^{r}$ para todo $I \in[k]^{(r)}$,

(ii) $\sigma^{L}(\mathcal{F}) \sim \sigma_{l} n^{k-l}$ para todo $L$ completo em $\mathcal{F}$ com $r \leq|L|=l<k$.

Então para todo $\epsilon>0$ e $n$ suficientemente grande pode-se selecionar um sistema $\mathcal{K}$ de $k$-ágonos de $\mathcal{F}$ tais que se pomos

$$
\mathcal{F}^{*}=\mathcal{F} \backslash\{F \in \mathcal{F}: \text { existe } K \in \mathcal{K}, F \in K\}=\mathcal{F} \backslash \bigcup_{K \in \mathcal{K}} K
$$

temos

(iii) $\rho_{I}\left(\mathcal{F}^{*}\right) \sim \rho n^{r} \exp \left(-\epsilon \sigma_{r}\right)$ para todo $I \in[k]^{(r)}$,

(iv) $\sigma^{L}\left(\mathcal{F}^{*}\right) \sim \sigma_{l} n^{k-l} \exp \left(-\epsilon \sigma_{r}\left(\left(\begin{array}{l}k \\ r\end{array}\right)-\left(\begin{array}{l}l \\ r\end{array}\right)\right)\right)$ para todo L completo em $\mathcal{F}^{*}(r \leq|L|=$ $l<k)$, 
(v) $\left|\left\{\left\{K^{1}, K^{2}\right\}: K^{1}, K^{2} \in \mathcal{K}, K^{1} \cap K^{2} \neq \emptyset\right\}\right| \leq 2 \epsilon \sigma_{r}\left|\bigcup_{K \in \mathcal{K}} K\right|$.

$\mathrm{O}$ sistema $\mathcal{K}$ é escolhido ao acaso, pondo um $k$-ágono de $\mathcal{F}$ em $\mathcal{K}$ com probabilidade $p=\epsilon / n^{k-r}$ e considerando estes eventos independentes. Para $I \in[k]^{(r)}$ mostra-se que a probabilidade de que uma aresta $F \in \mathcal{F}_{I}$, não coberta por $k$-ágonos, pertença a $\mathcal{F}_{I}^{*}$ é aproximadamente $\exp \left(-\epsilon \sigma_{r}\right)$ e daí obtêm-se (iii). A prova de (iv) é por indução. Para (v) estima-se a cardinalidade do conjunto usando-se a desigualdade de Markov.

Lema 3.3.3 (O algoritmo) Seja dado um r-grafo $k$-partido $\mathcal{F} \subset\left[\bigcup_{j=1}^{k} V_{j}\right]^{(r)}$, onde $\left|V_{j}\right|=$ $n$ para todo $j$, e suponha que $\rho$ e $\sigma_{l}(r \leq l \leq k-1)$ são reais positivos menores do que 1 tais que

(i) $\rho_{I}(\mathcal{F}) \sim \rho n^{r}$ para todo $I \in[k]^{(r)}$,

(ii) $\sigma^{L}(\mathcal{F}) \sim \sigma_{l} n^{k-l}$ para todo $L$ completo em $\mathcal{F}$ com $r \leq|L|=l<k$.

Então existe um sistema $\mathcal{K}$ de $k$-ágonos de $\mathcal{F}$ tais que

(iii) $\left|\bigcup_{K \in \mathcal{K}} K\right|=|\mathcal{F}|(1-o(1))$,

(iv) $|\mathcal{K}| \leq|\mathcal{F}|\left(\begin{array}{l}k \\ r\end{array}\right)^{-1}(1+o(1))$.

Para a prova, considera-se o seguinte algoritmo.

Algoritmo 3.3.4 Entrada: Um r-grafo k-partido $\mathcal{F}$ satisfazendo (i) e (ii) do Lema 2.1.3. Saída: Um sistema $\mathcal{K}$ de $k$-ágonos de $\mathcal{F}$ satisfazendo (iii) e (iv) do Lema 2.1.3.

1. Ponha $\left(V_{0}, \mathcal{F}_{0}\right)=(V, \mathcal{F})$ e $\epsilon=\delta / 4 \sigma_{r}$.

2. Para $s=0$ até $s=\left\lceil\left(1 / \epsilon \sigma_{r}\right) \log (2 / \delta)\right\rceil$ faça

Escolha $\mathcal{K}_{s}$ e $\mathcal{F}_{s}^{*}$ segundo o Lema 3.3.2.

Defina $\mathcal{F}_{s+1}=\mathcal{F}_{t}^{*}$.

3. Devolva $\mathcal{K}=\bigcup_{s=0}^{\left.\Gamma\left(1 / \epsilon \sigma_{r}\right) \log (2 / \delta)\right\rceil} \mathcal{K}_{s}$

Lema 3.3.5 (Invariantes) Em cada iteração do Algoritmo 3.3.4, temos

(i) $\sigma^{L}\left(\mathcal{F}_{s}\right) \sim \sigma_{l} n^{k-l} \exp \left(-\epsilon \sigma_{r} s\left(\left(\begin{array}{l}k \\ r\end{array}\right)-\left(\begin{array}{l}l \\ r\end{array}\right)\right)\right)$ para todo L completo em $\mathcal{F}_{s}$ e $r \leq|L|<k$,

(ii) $\rho_{I}\left(\mathcal{F}_{s}\right) \sim \rho n^{r} \exp \left(-\epsilon \sigma_{r}\right)$ para todo $I \in[k]^{(r)}$, 
(iii) $\left|\mathcal{K}_{s}\right| \leq\left(1+\frac{\delta}{2}\right)\left|\mathcal{F}-\mathcal{F}_{s}\right|\left(\begin{array}{l}k \\ r\end{array}\right)^{-1}$

Lema 3.3.6 (Corretude) $O$ sistema $\mathcal{K}$ de $k$-ágonos de $\mathcal{F}$ devolvido pelo Algoritmo 3.3.4 satisfaz (iii) e (iv) do Lema 2.1.3.

Observe que a estratégia do Algoritmo 3.3.4 é cobrir um pouco de $l$-conjuntos completos por $k$-ágonos, retirá-los, cobrir mais um pouco, retirá-los, etc.

No mesmo ano, Spencer [19], revisita o artigo de Rödl, esclarecendo um pouco mais a nova técnica; ele introduz certas noções de "regularidade" para sua versão do resultado de Rödl. Vamos tomar algumas de suas idéias básicas adaptando a discussão à nossa notação tentando conservar o espírito original.

Definição 3.3.7 Dizemos que um r-grafo $\mathcal{F}=(V, \mathcal{F})$ sobre $n$ vértices é quase-aleatório com densidade $\rho$ e tolerância $\epsilon$ se

(i) $|\mathcal{F}| \sim_{\epsilon} \rho\left(\begin{array}{l}n \\ r\end{array}\right)$

(ii) $\sigma^{F}(\mathcal{F}) \sim_{\epsilon} \rho^{\left(\begin{array}{l}k \\ r\end{array}\right)-1}\left(\begin{array}{l}n-r \\ k-r\end{array}\right)$ para todo $F \in \mathcal{F}$.

Observe que a classe de $r$-grafos para a qual foi provado o Lema 2.1.2 de Rödl está contida na classe de $r$-grafos quase aleatórios com densidade $\rho$ e tolerância $\epsilon$, sendo que a hipótese (ii) do Lema 2.1.2 é relaxada na classe maior. É essa a primeira aprimoração do resultado.

Lema 3.3.8 (A transformação) Sejam $\rho, \epsilon^{*}, \delta>0$ números reais positivos. Então existe $\epsilon>0$ e $n_{0}$ tal que dado um r-grafo quase-aleatório $\mathcal{F}=(V, \mathcal{F})$ sobre $n$ vértices, com densidade $\rho$ e tolerância $\epsilon$, existe uma família $\mathcal{K}$ de $k$-ágonos de $\mathcal{F}$ satisfazendo

$$
|\mathcal{K}| \sim_{\epsilon} \delta \rho\left(\begin{array}{l}
n \\
r
\end{array}\right)\left(\begin{array}{l}
k \\
r
\end{array}\right)^{-1}
$$

e tal que se $\mathcal{F}^{*}=\mathcal{F} \backslash \bigcup_{K \in \mathcal{K}} K$, então $\mathcal{F}^{*}$ é quase-aleatório com densidade $\rho \mathrm{e}^{-\delta}$ e tolerância $\epsilon^{*}$. Isto é

(i) $\left|\mathcal{F}^{*}\right| \sim_{\epsilon^{*}} \rho \mathrm{e}^{-\delta}\left(\begin{array}{l}n \\ r\end{array}\right)$,

(ii) $\sigma^{F}(\mathcal{F}) \sim_{\epsilon^{*}}\left(\rho \mathrm{e}^{-\delta}\right)^{\left(\begin{array}{l}k \\ r\end{array}\right)-1}\left(\begin{array}{l}n-r \\ k-r\end{array}\right)$ para todo $F \in \mathcal{F}$.

Não vamos entrar nos detalhes do artigo de Spencer, o qual é, na verdade, tangencial à discussão deste capítulo; em vez disso, vamos citar a "visão intuitiva" do seu $§ 1$, que cremos faz um bom resumo da técnica de Rödl:

Algoritmo 3.3.9 (Spencer, '85; [19]) 
"Sejam $r<k$ fixos e $n$ arbitrariamente grande. Seja $\mathrm{K}_{r}^{n}$ o $r$-grafo completo sobre o conjunto de vértices $[n]$. Seja $\delta$ um número real positivo arbitrariamente pequeno (fixamos $\delta$ primeiro e tomamos depois $n$ grande.) Seja $\mathcal{K}_{0}$ uma coleção aleatória de $\delta\left(\begin{array}{l}n \\ r\end{array}\right)\left(\begin{array}{l}k \\ r\end{array}\right)^{-1} k$ ágonos de $\mathrm{K}_{r}^{n}$ e seja $\mathcal{F}_{1}$ a família das $r$-hiperarestas de $\mathrm{K}_{r}^{n}$ que não estão contidas em nenhum $\mathrm{K}_{r}^{k} \in \mathcal{K}_{0}$. Já que $\mathcal{K}_{0}$ é $\delta$ vezes o tamanho de uma cobertura perfeita de $\mathrm{K}_{r}^{n}$ (se alguma existe), ele $\left(\mathcal{K}_{0}\right)$ cobriria, se (entre seus elementos) não há sobreposição, uma proporção $\delta$ das $r$-hiperarestas de $\mathrm{K}_{r}^{n}$. De fato, sobreposição é a consideração crítica. A hiperaresta típica é coberta uma média de $\delta$ vezes por $\mathcal{K}_{0}$. Existem muitos $k$-conjuntos cobrindo uma aresta dada e cada um deles tem só uma pequena chance de ser posto em $\mathcal{K}_{0}$. Portanto o número de $k$-conjuntos de $\mathcal{K}_{0}$ cobrindo uma $r$-hiperaresta dada é dado por uma distribuição Poisson com média $\delta$. Isto é, $\delta \mathrm{e}^{-\delta}$ das $r$-hiperarestas são cobertas exatamente uma vez, $\left(\delta^{2} / 2\right) \mathrm{e}^{-\delta}$ são cobertas exatamente duas vezes, $\left(\delta^{i} / i !\right) \mathrm{e}^{-\delta}$ são cobertas exatamente $i$ vezes e $\mathrm{e}^{-\delta}$ não são cobertas nenhuma vez e permanecem em $\mathcal{F}_{1}$. Quando $\delta$ é suficientemente pequeno, a proporção das $r$-hiperarestas cobertas duas ou mais vezes, grosseiramente $\delta^{2} / 2$, é uma proporção desprezível da proporção dos $r$ conjuntos, grosseiramente $\delta$, que são cobertos uma vez só. Isto é, $\mathcal{K}_{0}$ é uma excelente, embora não perfeita, cobertura de $\mathrm{K}_{r}^{n} \backslash \mathcal{F}_{1}$.

Continuamos o processo com $\mathcal{F}_{1}$. Escolhemos $\mathcal{K}_{1}$ entre os $k$-ágonos de $\mathcal{F}_{1}$. Isto é essencial pois não queremos que nenhuma das $\left(\begin{array}{l}k \\ r\end{array}\right) r$-hiperarestas cobertas por algum $\mathrm{K}_{r}^{k} \in$ $\mathcal{K}_{1}$ já esteja coberta por $\mathcal{K}_{0}$. Tomamos $\mathcal{K}_{1}$ ao acaso escolhendo a cardinalidade de modo que se não há sobreposição uma proporção $\delta$ de $\mathcal{F}_{1}$ será coberta. Seja $\mathcal{F}_{2}$ dado pelas $r$ hiperarestas restantes - aquelas não cobertas por nenhum $\mathrm{K}_{r}^{k} \in \mathcal{K}_{1}$. Novamente o número de $k$-conjuntos cobrindo uma $r$-hiperaresta de $\mathcal{F}_{1}$ é dado por uma distribuição Poisson com média $\delta$ e $\mathcal{K}_{1}$ é uma cobertura excelente de $\mathcal{F}_{1} \backslash \mathcal{F}_{2}$.

Iteramos este processo - dado $\mathcal{F}_{i}$ achamos $\mathcal{K}_{i}$ e pomos $\mathcal{F}_{i+1}$ igual às $r$-hiperarestas restantes - até atingir um $\mathcal{F}_{t}$ com uma proporção desprezível de $r$-hiperarestas. Já que cada $\left|\mathcal{F}_{i+1}\right| \sim \mathrm{e}^{-\delta}\left|\mathcal{F}_{i}\right|$ tomamos $t$ suficientemente grande de modo que $\mathrm{e}^{-\delta t}$ seja muito pequeno. Neste ponto as $r$-hiperarestas restantes são cobertas uma a uma. Embora isto seja muito custoso (queremos $k$-conjuntos $K$ para cobrir $\left(\begin{array}{l}k \\ r\end{array}\right)$ novas $r$-hiperarestas, mas aqui usamos um $k$-conjunto $K$ para cobrir uma $r$-hiperaresta) aceitamos tal custo já que $\left|\mathcal{F}_{t}\right|$ é pequeno. Com $\delta$ e $\mathrm{e}^{-\delta t}$ suficientemente pequenos a cobertura total tem uma proporção muito pequena de desperdício."

Naturalmente, a construção de uma tal cobertura era o objetivo de Rödl em [17] como veremos na Seção 2.4. A estratégia de cobrir uma a uma as hiperarestas restantes, quando ficam poucas por cobrir, vai ser um novo passo costumeiro nas provas algorítmicas dos sucessivos desenvolvimentos desta técnica.

Deixamos agora estas discussões iniciais para passar ao próximo passo no desenvolvimento desta técnica. 


\subsubsection{O teorema de Frankl e Rödl}

Ainda no mesmo ano, Frankl e Rödl [10] conseguem impor outras condições suficientes para a existência de uma cobertura quase perfeita num $r$-grafo; eles trocam as condições de regularidade que Spencer tinha imposto sobre o número de arestas do $r$-grafo e a quantidade de cliques contendo um conjunto completo por condições sobre o grau dos vértices. Examinemos rapidamente sua proposta.

Definição 3.3.10 Dado um hipergrafo $\mathcal{F}=(V, \mathcal{F})$ e $X \subset V$, definimos

$$
\mathcal{F}(X)=\{F \in \mathcal{F}: X \subset F\}
$$

$e$

$$
d(X)=|\mathcal{F}(X)|
$$

No caso de ser $X=\{x\}$, escrevemos simplesmente $\mathcal{F}(x)$ e d $(x)$ e se $X=\{x, y\}$ escreve$\operatorname{mos} \mathcal{F}(x, y)$ e d $(x, y)$.

Uma cobertura (de vértices por arestas ) $\mathcal{K}$ em $\mathcal{F}$ é um conjunto de hiperarestas de $\mathcal{F}$ tal que cada vértice está, no mínimo, numa hiperaresta de $\mathcal{K}$. Um empacotamento $\mathcal{P}$ em $\mathcal{F}$ é um conjunto de hiperarestas tal que todo vértice está, no máximo, numa hiperaresta de $\mathcal{P}$. Uma cobertura ou um empacotamento é perfeito se cada vértice está em exatamente uma de suas hiperarestas. Neste caso os conceitos coincidem. Denotamos por $\nu(\mathcal{F})$ a cardinalidade de um empacotamento máximo e por $\operatorname{Cob}(\mathcal{F})$ a cardinalidade de uma cobertura minima de $\mathcal{F}$.

Embora seja introduzida uma nova condição um pouco restritiva ((ii) abaixo), a nova transformação já não requer retirar um sistema de $k$-ágonos de $\mathcal{F}$ para que o $r$-grafo restante $\mathcal{F}^{*}$ satisfaça as teses, simplesmente retiramos um conjunto de arestas e definimos como o hipergrafo transformafo $\mathcal{F}^{*}$ o formado pelas arestas que não se intersectam com as arestas retiradas: mais uma simplificação.

Lema 3.3.11 (A transformação) Sejam dados $\epsilon>0$ e um $r$-grafo $\mathcal{F}=(V, \mathcal{F}$ ) sobre $n$ vértices tal que, para todo $x, y \in V$, dado $\epsilon>0$, existe $\rho=\rho(\epsilon)>0$ tal que se $\mathcal{F}$ satisfaz

(i) $|\mathcal{F}(x)| \sim_{\rho} D$,

(ii) $|\mathcal{F}(x, y)|<D /(\log n)^{a}$ para algum a $>3$ fixo,

então para $n>n_{0}(\epsilon, \rho)$ existe $\mathcal{R} \subset \mathcal{F}$ tal que

$$
\begin{gathered}
|\mathcal{R}| \sim_{2 \rho} \epsilon n / r, \\
|\bigcup \mathcal{R}| \sim_{4 \rho} n\left(1-\mathrm{e}^{-\epsilon}\right),
\end{gathered}
$$

e o r-grafo $\mathcal{F}^{*}=\{F \in \mathcal{F}: F \cap R=\emptyset$, para todo $R \in \mathcal{R}\}$ com vértices $V \backslash \bigcup \mathcal{R}$ satisfaz 
(iii) $\left|\mathcal{F}^{*}(x)\right| \sim_{6 \rho} D \mathrm{e}^{-\epsilon(r-1)}$,

(iv) $\left|\mathcal{F}^{*}(x, y)\right|<D \mathrm{e}^{-\epsilon(r-1)} /(\log n)^{a-o(1)}$.

O resultado central desta seção é o seguinte.

Teorema 3.3.12 (Frankl e Rödl, '85; [10]) Sejam dados $\epsilon>0$ arbitrário, um r-grafo $\mathcal{F}=(V, \mathcal{F})$ sobre $n$ vértices e a $>3$ um número real. Existe um número real positivo $\delta=\delta(\epsilon)$ tal que se para algum $D$ temos

(i) $d(x) \sim_{\delta} D$ para todo $x \in V$,

(ii) $d(x, y)<D /(\log n)^{a}$ para todo $x, y, x \neq y$,

então, para todo $n>n_{0}(\delta)$, temos

$$
\operatorname{Cob}(\mathcal{F}) \leq \frac{n}{r}(1+\epsilon)
$$

Para a prova considera-se o seguinte algoritmo.

Algoritmo 3.3.13 Entrada: $\epsilon>0, a>3$ e um r-grafo $\mathcal{F}$ satisfazendo (i) $e$ (ii) do Teorema 2.2.3.

Saída: Uma cobertura de $\mathcal{F}$ de tamanho $\leq \frac{n}{r}(1+\epsilon)$.

1. Ponha $\left(V_{0}, \mathcal{F}_{0}\right)=(V, \mathcal{F}), \rho=\epsilon / 3$ e $t$ o maior inteiro maior que $(\log r) / 2$ tal que $\sum_{s=0}^{t-1} \epsilon \frac{n}{r} \mathrm{e}^{-\epsilon s}+\mathrm{e}^{-\epsilon t} n \leq \frac{n}{r}(1+\epsilon)$

2. Para $s=0$ até $s=t-1$ faça

Calcule $\mathcal{R}_{s}$ e $\mathcal{F}_{s+1}=\mathcal{F}_{s}^{*}$ aplicando o Lema 3.3.11 a $\mathcal{F}_{s}$.

3. Para cada vértice não coberto por algum $\mathcal{R}_{s}$ escolha uma aresta contendo-o. Seja $\mathcal{R}_{t}$ o conjunto de tais arestas.

4. Devolva $\mathcal{R}=\bigcup_{s=0}^{t} \mathcal{R}_{s}$.

Lema 3.3.14 (Invariante) Em cada iteração do Algoritmo 3.3.13 temos

$$
\left|\mathcal{R}_{s+1}\right| \sim_{\epsilon / 3} \frac{\epsilon n}{r} \mathrm{e}^{-\epsilon s}
$$

A prova é feita por indução, usando no passo indutivo o Lema 2.2.2 e observando que em cada passo

$$
\left|V_{s+1}^{*}\right|=\left|\bigcup \mathcal{F}_{s+1}^{*}\right| \sim \mathrm{e}^{-\epsilon}\left|\bigcup \mathcal{F}_{s}\right|=\mathrm{e}^{-\epsilon}\left|V_{s}\right|
$$


Lema 3.3.15 (Corretude) $O$ conjunto $\mathcal{R}=\bigcup_{s=0}^{t} \mathcal{R}_{s}$ devolvido pelo Algoritmo 3.3.13 é uma cobertura de $\mathcal{F}$ e satisfaz

$$
|\mathcal{R}| \leq \frac{n}{r}(1+\epsilon)
$$

Na definição de $t$, os termos da somatória correspondem à cardinalidade dos conjuntos $\mathcal{R}_{s}$ do Passo 2 e como um caso particular da argumentação para o invariante, no final do passo do Algoritmo 3.3.13, temos que

$$
\left|V_{t}^{*}\right|=\left|\bigcup \mathcal{F}_{t}^{*}\right| \sim \mathrm{e}^{-\epsilon}\left|\bigcup \mathcal{F}_{t-1}\right|=\mathrm{e}^{-\epsilon}\left|V_{t-1}\right| \sim \mathrm{e}^{-\epsilon t}\left|V_{0}\right|=\mathrm{e}^{-\epsilon t} n
$$

é a quantidade de vértices que ficaram sem cobrir no Passo 2. Tais vértices são cobertos no Passo 3, e o Lema 2.2.6 segue da definição de $t$.

\subsubsection{A extensão de Pippenger e Spencer}

Alguns anos depois, em 1989, Pippenger e Spencer [16] obtêm um resultado bem mais forte que o de Frankl e Rödl, sob hipóteses menos restritivas. Parafraseando o sumário do artigo: Numa coleção de $r$-grafos com grau mínimo assintótico ao grau máximo $\Delta$ e tal que o número de hiperarestas contendo qualquer par de vértices fixos é desprezível em relação ao grau máximo, tem-se que o índice cromático é assintótico ao grau máximo. Isto quer dizer que as hiperarestas podem ser particionadas em empacotamentos ou coberturas quase-perfeitos.

A seguir apresentamos o novo resultado.

Definição 3.3.16 Dado um hipergrafo $\mathcal{F}=(V, \mathcal{F})$ o índice cromático $\chi^{\prime}(\mathcal{F})$ é o número minimo de empacotamentos nos quais as hiperarestas de $\mathcal{F}$ podem ser particionadas. Denotamos com $\phi(\mathcal{F})$ o tamanho máximo de uma coleção disjunta de coberturas de $\mathcal{F}$.

Teorema 3.3.17 (Pippenger e Spencer, '89; [16]) Para todo $r \geq 2$ e $\epsilon>0$ existem $\delta>0$ e $n_{0}$ tais que se um r-grafo $\mathcal{F}=(V, \mathcal{F})$ sobre $n$ vértices satisfaz

(i) $d(x) \sim_{\delta} \Delta(\mathcal{F})$ para todo $x \in V$,

(ii) $d(x, y) \leq \delta \Delta(\mathcal{F})$ para todo $x, y \in V$,

então

$$
\begin{aligned}
& \chi^{\prime}(\mathcal{F}) \sim_{\epsilon} \Delta(\mathcal{F}) \\
& \phi(\mathcal{F}) \sim_{\epsilon} \Delta(\mathcal{F})
\end{aligned}
$$

Observe como é mais geral a nova extensão: a condição (ii) do Teorema 2.3.2 inclui a classe de $r$-grafos que satisfaz (ii) do Teorema 2.2.3, e é uma condição bem mais simples. Veja como a extensão é mais forte: ela garante que podemos cobrir as arestas do $r$-grafo com $\Delta(\mathcal{F})$ coberturas, bastante mais do que a única cobertura devolvida pelo Teorema 2.2.3. 


\section{Referências Bibliográficas}

[1] M. Ajtai, J. Komlós, J. Pintz, J. Spencer, e E. Szemerédi. Extremal Uncrowded Hypergraphs. Journal of Combinatorial Theory, Series A, 32(3):321-335, 1982.

[2] M. Ajtai, J. Komlós, e E. Szemerédi. A Dense Infinite Sidon Sequence. European Journal of Combinatorics, 2(1):1-11, 1981.

[3] N. Alon, H. Lefman, e V. Rödl. On an Anti-Ramsey Type Result. Colloquia Mathematica Societatis János Bolyai, 60:10-22, 1991.

[4] S. Chowla. On a problem of Erdős e Turán in additive number theory. Proc.Natn.Acad.Sci.India, 14:1-2, 1944.

[5] R. A. Duke, H. Lefmann, e V. Rődl. On Uncrowded Hypergraphs. Reom Structures e Algorithms, 6(2 e 3):209-212, 1995.

[6] P. Erdős. On a problem of Sidon in additive number theory; addendum. Journal of the London Mathematical Society, 19:208, 1944.

[7] P. Erdős. Some of my Favourite Problems in Number Theory, Combinatorics, e Geometry. Resenhas do Instituto de Matemática e Estatística da USP, 2(2):165-196, 1995.

[8] P. Erdős e Hanani H. On a Limit Theorem in Combinatorial Analysis. Publ. Math. Debrecen, 10:10-13, 1963.

[9] P. Erdős e Turán. On a problem of sidon in additive number theory e some related problems. Journal of the London Mathematical Society, 16:212-215, 1941.

[10] P. Frankl e V. Rödl. Near Perfect Coverings in Graphs e Hypergraphs. European Journal of Combinatorics, 6(4):317-326, December 1985.

[11] Z. Füredi. Matchings e Covers in Hypergraphs. Graphs e Combinatorics, 4(2):115206, 1988.

[12] H. Halberstam e K. F. Roth. Sequences, volume I. Oxford University Press, Oxford, 1966. 
[13] J. H. Kim. The Ramsey Number $r(3, t)$ Has Order of Magnitude $t^{2} / \log t$. Reom Structures e Algorithms, 7(3):173-207, 1995.

[14] J. Komlós, J. Pintz, e E. Szemerédi. A Lower Bound for Heilbroon's Problem. Journal of the London Mathematical Society, 2(25):13-24, 1982.

[15] Krückeberg. $B_{2}$ Folgen und verwete Zahlenfolgen. Journal für reine und angewete Mathematik, Be 206:53-60, 1961.

[16] N. Pippenger e J. Spencer. Asymptotic Behavior of the Chromatic Index for Hypergraphs. Journal of Combinatorial Theory, Series A, 51(1):24-42, 1989.

[17] V. Rödl. On a Packing e Covering Problem. European Journal of Combinatorics, 6(1):69-78, 1985.

[18] K. F. Roth. On a Problem of Heilbronn. Appendix. Journal of the London Mathematical Society, 26:198-204, 1951.

[19] J. Spencer. Asymptotically Good Coverings. Pacific Journal of Mathematics, $118(2): 575-586,1985$. 